\title{
ON THE ALGEBRAIC K-THEORY OF THE COMPLEX K-THEORY SPECTRUM
}

\author{
CHRISTIAN AUSONI
}

\begin{abstract}
Let $p \geqslant 5$ be a prime, let $k u$ be the connective complex $K$-theory spectrum, and let $K(k u)$ be the algebraic $K$-theory spectrum of $k u$. In this paper we study the $p$ primary homotopy type of the spectrum $K(k u)$ by computing its mod $\left(p, v_{1}\right)$ homotopy groups. We show that up to a finite summand, these groups form a finitely generated free module over the polynomial algebra $\mathbb{F}_{p}[b]$, where $b$ is a class of degree $2 p+2$ defined as a "higher Bott element".
\end{abstract}

\section{INTRODUCTION}

The algebraic $K$-theory of a local or global number field $F$, with suitable finite coefficients, is known to satisfy a form of Bott periodicity. Bott periodicity refers here to the periodicity of topological complex $K$-theory, and is an example of $v_{1}$-periodicity in the sense of stable homotopy theory. For example, if $p$ is an odd prime and if $F$ contains a primitive $p$-th root of unity, then the $\bmod (p)$ algebraic $K$-theory $K_{*}(F ; \mathbb{Z} / p)$ of $F$ contains a non-nilpotent Bott element $\beta$ of degree 2 , with

$$
\beta^{p-1}=v_{1} .
$$

In one of its reformulations [19], [41], the Lichtenbaum-Quillen Conjecture asserts that the localization

$$
K_{*}(F ; \mathbb{Z} / p) \rightarrow K_{*}(F ; \mathbb{Z} / p)\left[\beta^{-1}\right]
$$

away from $\beta$ is an isomorphism in positive degrees. In particular, $K_{*}(F ; \mathbb{Z} / p)$ is periodic of period 2 in positive degrees. In the local case, this follows from [23, Theorem D].

The $p$-local stable homotopy category also features higher forms of periodicity [25], one for each integer $n \geqslant 0$, referred to as $v_{n}$-periodicity. It is detected for example by the $n$th Morava $K$-theory $K(n)$, having coefficients $K(0)_{*}=\mathbb{Q}$ and $K(n)_{*}=\mathbb{F}_{p}\left[v_{n}, v_{n}^{-1}\right]$ with $\left|v_{n}\right|=2 p^{n}-2$ if $n \geqslant 1$. The study of $v_{2}$-periodicity is at the focus of current research in algebraic topology, as illustrated for example by the efforts to define the elliptic cohomology theory known as topological modular forms [24].

Waldhausen [44] extended the definition of algebraic $K$-theory to include specific "rings up to homotopy" called structured ring spectra, like $E_{\infty}$ ring spectra [30], $S$-algebras [20], or symmetric ring-spectra [26]. The chromatic red-shift conjecture [4] of John Rognes predicts that the algebraic $K$-theory of a suitable $v_{n}$-periodic structured ring-spectrum is essentially $v_{n+1}$-periodic, as illustrated above in the case of number fields (which are $v_{0}$-periodic). For an example with the next level of periodicity, we consider the algebraic $K$-theory of topological $K$-theory.

2000 Mathematics Subject Classification. 19D55, 55N15.

Published version DOI : 10.1007/s00222-010-0239-x.

Research supported in part by the Institute Mittag-Leffler, Djursholm, and the Max Planck Institute for Mathematics, Bonn. 
Let $p \geqslant 5$ be a prime, and let $k u_{p}$ denote the $p$-completed connective complex $K$-theory spectrum with coefficients $k u_{p_{*}}=\mathbb{Z}_{p}[u],|u|=2$, where $\mathbb{Z}_{p}$ is the ring of $p$-adic integers. Let $\ell_{p}$ be the Adams summand of $k u_{p}$ with coefficients $\ell_{p_{*}}=\mathbb{Z}_{p}\left[v_{1}\right]$ and $v_{1}=u^{p-1}$. In joint work with John Rognes [3], we have computed the $\bmod \left(p, v_{1}\right)$ algebraic $K$-theory of the $S$-algebra $\ell_{p}$, denoted $V(1)_{*} K\left(\ell_{p}\right)$, and we have shown that it is essentially $v_{2}$-periodic. This computation provides a first example of red-shift for non-ordinary rings.

In this paper, following the discussion in [1, Section 10], we interpret $k u_{p}$ as a tamely ramified extension of $\ell_{p}$ of degree $p-1$, and we compute $V(1)_{*} K\left(k u_{p}\right)$. As expected, the result is again essentially periodic. However, $V(1)_{*} K\left(k u_{p}\right)$ has a shorter period: its periodicity is given by multiplication with a higher Bott element $b \in V(1)_{*} K\left(k u_{p}\right)$, of degree $2 p+2$. We defer a definition of $b$ to Section 3 below, and summarize our main result in the following statement.

Theorem 1.1. Let $p \geqslant 5$ be a prime. The higher Bott element $b \in V(1)_{2 p+2} K\left(k u_{p}\right)$ is non-nilpotent and satisfies the relation

$$
b^{p-1}=-v_{2} .
$$

Let $P(b)$ denote the polynomial $\mathbb{F}_{p}$-sub-algebra of $V(1)_{*} K\left(k u_{p}\right)$ generated by $b$. Then there is a short exact sequence of graded $P(b)$-modules

$$
0 \rightarrow \Sigma^{2 p-3} \mathbb{F}_{p} \rightarrow V(1)_{*} K\left(k u_{p}\right) \rightarrow F \rightarrow 0,
$$

where $\Sigma^{2 p-3} \mathbb{F}_{p}$ is the sub-module of b-torsion elements and $F$ is a free $P(b)$-module on $8+4(p-1)$ generators.

A detailed description of the free $P(b)$-module $F$ is given in Theorem 8.1. The proof is based on evaluating the cyclotomic trace map [11]

$$
\operatorname{trc}: K\left(k u_{p}\right) \rightarrow T C\left(k u_{p}\right)
$$

to topological cyclic homology. We emphasize that the higher Bott element $b$ is not the reduction of a class in the mod $(p)$ or integral homotopy of $K\left(k u_{p}\right)$.

The cyclic subgroup $\Delta \subset \mathbb{Z}_{p}^{\times}$of order $p-1$ acts on $k u_{p}$ by $p$-adic Adams operations. The Adams summand is defined as the homotopy fixed-point spectrum $\ell_{p}=k u_{p}^{h \Delta}$, and $\Delta$ qualifies as the Galois group of the tamely ramified extension $\ell_{p} \rightarrow k u_{p}$ of commutative $S$ algebras given by the inclusion of homotopy fixed-points. We proved in [1, Theorem 10.2] that the induced map $K\left(\ell_{p}\right) \rightarrow K\left(k u_{p}\right)$ factors through a weak equivalence

$$
K\left(\ell_{p}\right) \stackrel{\simeq}{\longrightarrow} K\left(k u_{p}\right)^{h \Delta}
$$

after $p$-completion. The $\bmod \left(p, v_{1}\right)$ homotopy groups of $K\left(\ell_{p}\right)$ and $K\left(k u_{p}\right)$ are related as follows.

Proposition 1.2. Let $i_{*}: V(1)_{*} K\left(\ell_{p}\right) \rightarrow V(1)_{*} K\left(k u_{p}\right)$ be the homomorphism induced by the extension of $S$-algebras $\ell_{p} \rightarrow k u_{p}$.

(a) The homomorphism $i_{*}$ factors through an isomorphism

$$
V(1)_{*} K\left(\ell_{p}\right) \cong\left(V(1)_{*} K\left(k u_{p}\right)\right)^{\Delta} \subset V(1)_{*} K\left(k u_{p}\right)
$$

onto the classes fixed by the Galois group. The higher Bott element $b$ is not fixed under the action of $\Delta$, but $b^{p-1}=-v_{2}$ is, accounting for the $v_{2}$-periodicity of $V(1)_{*} K\left(\ell_{p}\right)$. 
(b) The homomorphism

$$
\mu: P(b) \otimes_{P\left(v_{2}\right)} V(1)_{*} K\left(\ell_{p}\right) \rightarrow V(1)_{*} K\left(k u_{p}\right)
$$

induced by $i_{*}$ and the $P(b)$-action has finite kernel and cokernel, and is an isomorphism in degrees larger than $2 p^{2}-4$. By localizing away from $b$, we obtain an isomorphism of $P\left(b, b^{-1}\right)$-modules

$$
P\left(b, b^{-1}\right) \otimes_{P\left(v_{2}\right)} V(1)_{*} K\left(\ell_{p}\right) \stackrel{\cong}{\longrightarrow} V(1)_{*} K\left(k u_{p}\right)\left[b^{-1}\right] .
$$

In particular, the $P(b)$-module $V(1)_{*} K\left(k u_{p}\right)$ is almost the module obtained from the $P\left(v_{2}\right)$-module $V(1)_{*} K\left(\ell_{p}\right)$ by the extension $P\left(v_{2}\right) \subset P(b)$ of scalars. The kernel of $\mu$ consists of $b$-multiples of the $v_{2}$-torsion elements, and we have a non-trivial cokernel because some of the $P\left(v_{2}\right)$-module generators of $V(1)_{*} K\left(\ell_{p}\right)$ are multiples of $b$ in $V(1)_{*} K\left(k u_{p}\right)$, see Corollary 8.2 .

Notice that for the cyclotomic extension $\mathbb{Z}_{p} \rightarrow \mathbb{Z}_{p}\left[\zeta_{p}\right]$ of complete discrete valuation rings with Galois group $\Delta$ (where $\zeta_{p}$ is a primitive $p$ th root of unity), we have corresponding results in mod $(p)$ algebraic $K$-theory. In effect, the natural homomorphism $K_{*}\left(\mathbb{Z}_{p} ; \mathbb{Z} / p\right) \rightarrow K_{*}\left(\mathbb{Z}_{p}\left[\zeta_{p}\right] ; \mathbb{Z} / p\right)$ factors through an isomorphism onto the $\Delta$-fixed classes. The Bott class $\beta \in K_{2}\left(\mathbb{Z}_{p}\left[\zeta_{p}\right] ; \mathbb{Z} / p\right)$ is not fixed under $\Delta$, but $\beta^{p-1}=v_{1}$ is. This accounts for the fact that $K_{*}\left(\mathbb{Z}_{p}\left[\zeta_{p}\right] ; \mathbb{Z} / p\right)$ has a shorter period than $K_{*}\left(\mathbb{Z}_{p} ; \mathbb{Z} / p\right)$. Moreover, the $P(\beta)$-module $K_{*}\left(\mathbb{Z}_{p}\left[\zeta_{p}\right] ; \mathbb{Z} / p\right)$ is essentially obtained from the $P\left(v_{1}\right)$-module $K_{*}(\mathbb{Z} p ; \mathbb{Z} / p)$ by the extension $P\left(v_{1}\right) \subset P(\beta)$ of scalars. These facts are extracted from computations by Hesselholt and Madsen [23, Theorem D]. We therefore interpret Proposition 1.2 as follows : up to a chromatic shift of one in the sense of stable homotopy theory, the algebraic $K$-theory spectra of the tamely ramified extensions

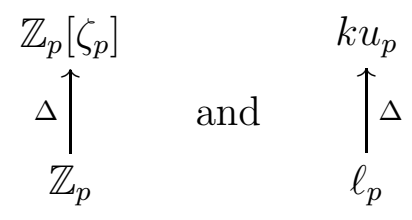

have a comparable formal structure.

This example of red-shift provides evidence that structural results for the algebraic $K$ theory of ordinary rings might well be generalized to provide more conceptual descriptions of the algebraic $K$-theory of $S$-algebras. See Remarks 3.5 and 8.4 for a discussion of the results we have in mind here.

We now turn to the algebraic $K$-theory $K(k u)$ of the (non $p$-completed) connective complex $K$-theory spectrum $k u$, with coefficients $k u_{*}=\mathbb{Z}[u],|u|=2$. The $p$-completion $k u \rightarrow k u_{p}$ induces a map

$$
\kappa: K(k u) \rightarrow K\left(k u_{p}\right),
$$

and the higher Bott element $b \in V(1)_{2 p+2} K\left(k u_{p}\right)$ is in fact defined as the image of a class with same name in $V(1)_{2 p+2} K(k u)$. The difference between $K(k u)$ and $K\left(k u_{p}\right)$ can be measured by means of the homotopy Cartesian square after $p$-completion

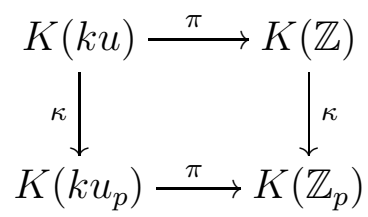


of Dundas [18, page 224]. Here $\pi$ denotes the map induced in $K$-theory by the zeroth Postnikov sections $k u \rightarrow H \mathbb{Z}$ and $k u_{p} \rightarrow H \mathbb{Z}_{p}$, where $H R$ is the Eilenberg-Mac Lane spectrum of the ring $R$. The homotopy type of the $p$-completion of $K\left(\mathbb{Z}_{p}\right)$ has been computed by Bökstedt, Hesselholt and Madsen [22,,[13]. The Lichtenbaum-Quillen Conjecture for $K(\mathbb{Z})$ (see for example [34, §6]) implies that the homotopy fiber of $K(\mathbb{Z}) \rightarrow K(\mathbb{Z} p$ ) has finite $V(1)$-homotopy groups, which are concentrated in degrees smaller than $2 p-1$. This implies the result below. In fact there seems to be some consensus that work of Vladimir Voevodsky and Markus Rost should imply the Lichtenbaum-Quillen Conjecture, but to our knowledge this has not appeared in written form. We therefore keep it as an assumption in the following results.

Proposition 1.3. Let $p \geqslant 5$ be a prime, and assume that the Lichtenbaum-Quillen Conjecture for $K(\mathbb{Z})$ holds at $p$. Then the homomorphism of $P(b)$-modules

$$
\kappa_{*}: V(1)_{*} K(k u) \rightarrow V(1)_{*} K\left(k u_{p}\right)
$$

is an isomorphism in degrees larger than $2 p-1$. Localizing the V(1)-homotopy groups away from $b$, we obtain an isomorphism

$$
V(1)_{*} K(k u)\left[b^{-1}\right] \cong V(1)_{*} K\left(k u_{p}\right)\left[b^{-1}\right]
$$

of $P\left(b, b^{-1}\right)$-algebras.

This result is of interest beyond algebraic $K$-theory. Baas, Dundas and Rognes have proposed a geometric definition of a cohomology theory derived from a suitable notion of bundles of complex two-vector spaces [7]. These are a two-categorical analogue of the ordinary complex vector bundles which enter in the geometric definition of topological $K$-theory. They conjectured in [7, 5.1] that the spectrum representing this new theory is weakly homotopy equivalent to $K(k u)$, and this was proved by these authors and Birgit Richter in [6]. The next statement follows from Theorem 1.1 and Proposition [1.3.

Proposition 1.4. If the Lichtenbaum-Quillen Conjecture for $K(\mathbb{Z})$ holds, then at any prime $p \geqslant 5$ the spectrum $K(k u)$ is of telescopic complexity two in the sense of [7, 6.1].

This result was anticipated in [7, §6], and ensures that the cohomology theory derived from two-vector bundles is, from the view-point of stable homotopy theory, a legitimate candidate for elliptic cohomology.

The computations presented in this paper fail at the primes 2 and 3 , because of the nonexistence of the ring-spectrum $V(1)$. Theoretically, computations in $\bmod (p)$ homotopy or in integral homotopy could also be carried out, but the algebra seems quite intractable. Another approach [16], 28] is via homology computations. There are ongoing projects in this direction by Robert Bruner, Sverre Lunøe-Nielsen and John Rognes.

Up to degree three, the integral homotopy groups of $K(k u)$ can be computed essentially by using the map $\pi: K(k u) \rightarrow K(\mathbb{Z})$ introduced above. The map $\pi_{*}: K_{*}(k u) \rightarrow K_{*}(\mathbb{Z})$ is 3 -connected, so that

$$
K_{0}(k u) \cong \mathbb{Z}, K_{1}(k u) \cong \mathbb{Z} / 2 \text { and } K_{2}(k u) \cong \mathbb{Z} / 2 .
$$

Here $K_{1}(k u)$ and $K_{2}(k u)$ are generated by the image of $\eta \in \pi_{1} S$ and $\eta^{2} \in \pi_{2} S$, respectively, under the unit $S \rightarrow K(k u)$. Let $w: B B U_{\otimes} \rightarrow \Omega^{\infty} K(k u)$ be the map induced by the inclusion of units, see (3.3) . There is a non-split extension

$$
0 \rightarrow \pi_{3}\left(B B U_{\otimes}\right) \stackrel{w_{*}}{\longrightarrow} K_{3}(k u) \stackrel{\pi_{*}}{\longrightarrow} K_{3}(\mathbb{Z}) \rightarrow 0
$$


with $\pi_{3}\left(B B U_{\otimes}\right) \cong \mathbb{Z}\{\mu\}, K_{3}(k u) \cong \mathbb{Z}\{\varsigma\} \oplus \mathbb{Z} / 24\{\nu\}$ and $K_{3}(\mathbb{Z}) \cong \mathbb{Z} / 48\{\lambda\}$, where $\nu$ is the image of the Hopf class $\nu$, which generates $\pi_{3} S \cong \mathbb{Z} / 24$. We have $w_{*}(\mu)=2 \varsigma-\nu$ and $\pi_{*}(\varsigma)=\lambda$. See [2] for details. This indicates that the integral homotopy groups $K_{*}(k u)$ contain intriguing non-trivial extensions from subgroups in $\pi_{*} S, \pi_{*} B B U_{\otimes}$ and $K_{*}(\mathbb{Z})$.

The rational algebraic $K$-groups of $k u$ are well understood. In joint work with John Rognes [5], we have proved that after rationalization, the sequence

$$
B B U_{\otimes} \stackrel{w}{\longrightarrow} \Omega^{\infty} K(k u) \stackrel{\pi}{\longrightarrow} \Omega^{\infty} K(\mathbb{Z})
$$

is a split homotopy fibre-sequence. A rational splitting of $w$ is provided by a rational determinant map $\Omega^{\infty} K(k u) \rightarrow\left(B B U_{\otimes}\right)_{\mathbb{Q}}$. In particular, by Borel's computation [14] of $K_{*}(\mathbb{Z}) \otimes \mathbb{Q}$, there is a rational equivalence

$$
\Omega^{\infty} K(k u) \simeq_{\mathbb{Q}} S U \times(S U / S O) \times \mathbb{Z} .
$$

All but finitely many of the non-torsion classes in the integral homotopy groups $\pi_{*} K(k u)$ detected by this equivalence reduce $\bmod (p)$ to multiples of $v_{1}$, and hence reduce to zero in $V(1)_{*} K(k u)$.

We briefly discuss the contents of this paper. In Section 2, we study the $V(1)$-homotopy of the Eilenberg-Mac Lane space $K(\mathbb{Z}, 3)$, which is a subspace of the space of units of $k u$. In Section 3, we define low-dimensional classes in $V(1)_{*} K(k u)$ corresponding to units of $k u$, and in particular we introduce the higher Bott element. We prove in Section 4 that these classes are non-zero by means of the Bökstedt trace map

$$
\operatorname{tr}: K(k u) \rightarrow T H H(k u)
$$

to topological Hochschild homology. In Section 5, we compute $V(1)_{n} K\left(k u_{p}\right)$ for $n \leqslant$ $2 p-2$. This complements the computations in higher degrees provided by the cyclotomic trace

$$
\operatorname{trc}: K\left(k u_{p}\right) \rightarrow T C\left(k u_{p}\right)
$$

to topological cyclic homology. In Section [6 we compute the various homotopy fixed points of $T H H\left(k u_{p}\right)$ under the action of the cyclic groups $C_{p^{n}}$ and the circle, which are the ingredients for the computation of $V(1)_{*} T C\left(k u_{p}\right)$ in Section 7 . In Section 8 we prove Theorem 1.1 on the structure of $V(1)_{*} K\left(k u_{p}\right)$ stated above. We also give a computation of $V(1)_{*} K\left(K U_{p}\right)$ for $K U_{p}$ the $p$-completed periodic $K$-theory spectrum, up to some indeterminacy.

Notations and conventions. Throughout the paper, unless stated otherwise, $p$ will be a fixed prime with $p \geqslant 5$, and $\mathbb{Z}_{p}$ will denote the $p$-adic integers. For an $\mathbb{F}_{p}$-vector space $V$, let $E(V), P(V)$ and $\Gamma(V)$ be the exterior algebra, polynomial algebra and divided power algebra on $V$, respectively. If $V$ has a basis $\left\{x_{1}, \ldots, x_{n}\right\}$, we write $V=\mathbb{F}_{p}\left\{x_{1}, \ldots, x_{n}\right\}$ and $E\left(x_{1}, \ldots, x_{n}\right), P\left(x_{1}, \ldots, x_{n}\right)$ and $\Gamma\left(x_{1}, \ldots, x_{n}\right)$ for these algebras. By definition, $\Gamma(x)$ is the $\mathbb{F}_{p^{-}}$-vector space $\mathbb{F}_{p}\left\{\gamma_{k} x \mid k \geqslant 0\right\}$ with product given by $\gamma_{i} x \cdot \gamma_{j} x=\left(\begin{array}{c}i+j \\ i\end{array}\right) \gamma_{i+j} x$, where $\gamma_{0} x=1$ and $\gamma_{1} x=x$. Let $P_{h}(x)=P(x) /\left(x^{h}\right)$ be the truncated polynomial algebra of height $h$. For an algebra $A$, we denote by $A\left\{x_{1}, \ldots, x_{n}\right\}$ the free $A$-module generated by $x_{1}, \ldots, x_{n}$.

If $Y$ is a space and $E_{*}$ is a homology theory, such as mod $(p)$ homology, $V(1)$-homotopy or Morava $K$-theory $K(2)_{*}$, we denote by $E_{*}(Y)$ the unreduced $E_{*}$-homology of $Y$, which we identify with the $E_{*}$-homology of the suspension spectrum $\Sigma^{\infty}\left(Y_{+}\right)$, where $Y_{+}$denotes $Y$ with a disjoint base-point added. We usually write $\Sigma_{+}^{\infty} Y$ instead of $\Sigma^{\infty}\left(Y_{+}\right)$. 
The reduced $E_{*}$-homology of a pointed space $X$ is denoted $\widetilde{E}_{*}(X)$. We denote $\pi_{*} X$ the (unstable) homotopy groups of $X$, and $\pi_{*} \Sigma^{\infty} X$ its stable homotopy groups.

If $f: A \rightarrow B$ is a map of $S$-algebras, we also denote by $f$ its image under various functors like $T H H, T C$ or $K$.

In our computations with spectral sequences, we often determine a differential $d$ only up to multiplication by a unit. We use the notation $d(x) \doteq y$ to indicate that the equation $d(x)=\alpha y$ holds for some unit $\alpha \in \mathbb{F}_{p}$. Classes surviving to the $E^{r}$-term of a spectral sequence, for $r \geqslant 3$, are often given as a product of classes in the $E^{2}$-term. To improve the readability, we denote the product of two classes $x, y$ in $E^{r}$ by $x \cdot y$.

\section{ON THE $V(1)$-HOмотору OF $K(\mathbb{Z}, 3)$}

If $G$ is a topological monoid, let us denote by $B G$ its classifying space, obtained by realization of the bar construction, see for example [36, §1]. If $G$ is an Abelian topological group, then so is $B G$. The space $B G$ is equipped with the bar filtration

$$
\{*\}=B_{0} \subset B_{1} \subset B_{2} \subset \cdots \subset B_{n-1} \subset B_{n} \subset \ldots B G,
$$

with filtration quotients $B_{n} / B_{n-1} \cong \Sigma^{n}\left(G^{\wedge n}\right)$. In particular, we have a map

$$
s: \Sigma G=B_{1} \subset B G,
$$

which in any homology theory $E_{*}$ induces a map

$$
\sigma: E_{*} G \rightarrow E_{*+1} B G
$$

called the suspension. If $E_{*}$ is a multiplicative homology theory satisfying the Künneth isomorphism, we have the bar spectral sequence [36, §2]

$$
\begin{aligned}
& E_{s, *}^{1}(G)=\widetilde{E}_{*}(G)^{\otimes_{E_{*}} s}, \\
& E_{s, t}^{2}(G)=\operatorname{Tor}_{s, t}^{E_{*}(G)}\left(E_{*}, E_{*}\right) \Rightarrow E_{s+t}(B G)
\end{aligned}
$$

associated to the bar filtration (2.1).

Let $K(\mathbb{Z}, 0)$ be equal to $\mathbb{Z}$ as a discrete topological group, and for $m \geqslant 1$, we define recursively the Eilenberg-Mac Lane space $K(\mathbb{Z}, m)$ as the Abelian topological group $B K(\mathbb{Z}, m-1)$. We recall Cartan's computation of the algebra $H_{*}\left(K(\mathbb{Z}, m) ; \mathbb{F}_{p}\right)$ for $p$ an odd prime and $m=2,3$. The generators are constructed explicitly from the unit $1 \in H_{*}\left(K(\mathbb{Z}, 0) ; \mathbb{F}_{p}\right)$ by means of the suspension $\sigma$ and two further operators

$$
\begin{aligned}
& \varphi: H_{2 q}\left(K(\mathbb{Z}, m) ; \mathbb{F}_{p}\right) \rightarrow H_{2 p q+2}\left(K(\mathbb{Z}, m+1) ; \mathbb{F}_{p}\right) \text { and } \\
& \gamma_{p}: H_{2 q}\left(K(\mathbb{Z}, m) ; \mathbb{F}_{p}\right) \rightarrow H_{2 p q}\left(K(\mathbb{Z}, m) ; \mathbb{F}_{p}\right),
\end{aligned}
$$

called the transpotence [17, page 6-06] and the $p$-th divided power [17, page 7-07], respectively. The transpotence is an additive homomorphism since $p$ is odd. For $x \in$ $H_{2 q}\left(K(\mathbb{Z}, m) ; \mathbb{F}_{p}\right)$, the class $\varphi(x)$ is represented, for example, by

$$
x^{p-1} \otimes x \in E_{2,2 p q}^{1}(K(\mathbb{Z}, m))
$$

in the bar spectral sequence. The algebra $H_{*}\left(K(\mathbb{Z}, m) ; \mathbb{F}_{p}\right)$ has the structure of an algebra with divided powers, which are uniquely determined by $\gamma_{p}$.

Theorem 2.1 (Cartan). Let $p$ be an odd prime. There are isomorphisms of $\mathbb{F}_{p}$-algebras with divided powers

$$
\Gamma(y) \stackrel{\cong}{\longrightarrow} H_{*}\left(K(\mathbb{Z}, 2) ; \mathbb{F}_{p}\right)
$$


given by $y \mapsto \sigma \sigma(1)$, with $|y|=2$, and

$$
\bigotimes_{k \geqslant 0} E\left(e_{k}\right) \otimes \Gamma\left(f_{k}\right) \stackrel{\cong}{\longrightarrow} H_{*}\left(K(\mathbb{Z}, 3) ; \mathbb{F}_{p}\right)
$$

given by $e_{k} \mapsto \sigma \gamma_{p}^{k} \sigma \sigma(1)$ and $f_{k} \mapsto \varphi \gamma_{p}^{k} \sigma \sigma(1)$, with degrees $\left|e_{k}\right|=2 p^{k}+1$ and $\left|f_{k}\right|=$ $2 p^{k+1}+2$. For $k \geqslant 0$, the generators $f_{k}$ and $e_{k+1}$ are related by a primary mod $(p)$ homology Bockstein

$$
\beta\left(f_{k}\right)=e_{k+1} .
$$

Proof. The computation of $H_{*}\left(K(\mathbb{Z}, m) ; \mathbb{F}_{p}\right)$ as an algebra is given in [17, Théorème fondamental, p. 9-03]. The Bockstein relation $\beta\left(f_{k}\right)=e_{k+1}$ is established in [17, page 804].

Ravenel and Wilson [36] make use of the bar spectral sequence to compute the Morava $K$-theory $K(n)_{*} K(\pi, m)$ as an algebra when $\pi=\mathbb{Z}$ or $\mathbb{Z} / p^{j}$. All generators can be defined explicitly, starting with the unit $1 \in K(n)_{*} K(\pi, 0)$ and using the suspension, divided powers, transpotence and the Hopf-ring structure on $K(n)_{*} K(\pi, *)$. We refer to [36, 5.6 and 12.1] for the following result, and for the definition of the generators $\beta_{(k)}$ and $b_{(2 k, 1)}$.

Theorem 2.2 (Ravenel-Wilson). Let $p \geqslant 3$ be a prime and let $K(2)$ be the Morava $K$ theory spectrum with coefficients $K(2)_{*}=\mathbb{F}_{p}\left[v_{2}, v_{2}^{-1}\right]$. There are isomorphisms of $K(2)_{*}$ algebras

$$
K(2)_{*} K(\mathbb{Z}, 2) \cong K(2)_{*}\left[\beta_{(k)} \mid k \geqslant 0\right] /\left(\beta_{(0)}^{p}, \beta_{(k+1)}^{p}-v_{2}^{p^{k}} \beta_{(k)} \mid k \geqslant 0\right)
$$

where $\left|\beta_{(k)}\right|=2 p^{k}$, and

$$
K(2)_{*} K(\mathbb{Z}, 3) \cong K(2)_{*}\left[b_{(2 k, 1)} \mid k \geqslant 0\right] /\left(b_{(2 k, 1)}^{p}+v_{2}^{p^{k}} b_{(2 k, 1)} \mid k \geqslant 0\right)
$$

where $\left|b_{(2 k, 1)}\right|=2 p^{k}(p+1)$. The class $\beta_{(0)} \in K(2)_{2} K(\mathbb{Z}, 2)$ is equal to $\sigma \sigma(1)$, and the class $b_{(0,1)} \in K(2)_{2 p+2} K(\mathbb{Z}, 3)$ is the transpotence of $\beta_{(0)}$.

We now turn to $V(1)$-homotopy. For an integer $n \geqslant 0$, we denote by $V(n)$ the SmithToda complex [42, with mod $(p)$ homology given by

$$
H_{*}\left(V(n) ; \mathbb{F}_{p}\right) \cong E\left(\tau_{0}, \ldots, \tau_{n}\right)
$$

as a left sub-comodule of the dual Steenrod algebra. In particular, $V(0)=S / p$ is the $\bmod (p)$ Moore spectrum, and the spectra $V(0)$ and $V(1)$ fit in cofibre sequences

$$
S \stackrel{p}{\longrightarrow} S \stackrel{i_{0}}{\longrightarrow} V(0) \stackrel{j_{0}}{\longrightarrow} \Sigma S
$$

and

$$
\Sigma^{2 p-2} V(0) \stackrel{v_{1}}{\longrightarrow} V(0) \stackrel{i_{1}}{\longrightarrow} V(1) \stackrel{j_{1}}{\longrightarrow} \Sigma^{2 p-1} V(0),
$$

where $v_{1}$ is a periodic map. For $n=0,1$ and $p \geqslant 5$, the spectrum $V(n)$ is a commutative ring spectrum [35], and its ring of coefficients $V(n)_{*}$ is an $\mathbb{F}_{p}$-algebra which contains a non-nilpotent class $v_{n+1}$, of degree $2 p^{n+1}-2$. We call " $V(n)$-homotopy" the homology theory associated to the spectrum $V(n)$. In other words, the $V(n)$-homotopy groups of a spectrum $X$ are defined by

$$
V(n)_{*} X=\pi_{*}(V(n) \wedge X)
$$


Notice that $V(0)_{*} X$ is denoted $\pi_{*}(X ; \mathbb{Z} / p)$ by some authors, and called the mod $(p)$ homotopy groups of $X$. By analogy, we sometimes call $V(1)_{*} X$ the $\bmod \left(p, v_{1}\right)$ homotopy groups of $X$. If $Y$ is a space, then $V(n)_{*} Y$ is defined as $V(n)_{*} \Sigma_{+}^{\infty} Y$.

The primary mod $(p)$ homotopy Bockstein $\beta_{0,1}: V(0)_{*} X \rightarrow V(0)_{*-1} X$ is the homomorphism induced by $\left(\Sigma i_{0}\right) j_{0}$, and the primary mod $\left(v_{1}\right)$ homotopy Bockstein $\beta_{1,1}$ : $V(1)_{*} X \rightarrow V(1)_{*-2 p+1} X$ is the homomorphism induced by $\left(\Sigma^{2 p-1} i_{1}\right) j_{1}$. The homomorphisms $i_{0 *}: \pi_{*}(X) \rightarrow V(0)_{*} X$ and $i_{1 *}: V(0)_{*} X \rightarrow V(1)_{*} X$ are called the mod $(p)$ reduction and the mod $\left(v_{1}\right)$ reduction, respectively.

Let $H \mathbb{F}_{p}$ be the Eilenberg-Mac Lane spectrum of $\mathbb{F}_{p}$. The unit map $S \rightarrow H \mathbb{F}_{p}$ factors through a map of ring spectra $h: V(1) \rightarrow H \mathbb{F}_{p}$, which induces an injective homomorphism in $\bmod (p)$ homology. Identifying the homology of $V(1)$ with its image in the dual Steenrod algebra $A_{*}$, we obtain the isomorphism

$$
H_{*}\left(V(1) ; \mathbb{F}_{p}\right) \cong E\left(\tau_{0}, \tau_{1}\right)
$$

of left $A_{*}$-comodule algebras mentioned above. Toda [42, Theorem 5.2] computed $V(1)_{*}$ in a range of degrees for which the Adams spectral sequence collapses. Up to some renaming of the classes, we deduce from his theorem that for $p \geqslant 5$ there is an isomorphism of $P\left(v_{2}\right) \otimes P\left(\beta_{1}\right)$-modules

$$
P\left(v_{2}\right) \otimes P\left(\beta_{1}\right) \otimes \mathbb{F}_{p}\left\{1, \alpha_{1}, \beta_{1}^{\prime},\left(\alpha_{1} \beta_{1}\right)^{\sharp}\right\} \rightarrow V(1)_{*}
$$

in degrees $*<4 p^{2}-2 p-4$. The classes $\alpha_{1}$ and $\beta_{1}$ are the $\bmod \left(p, v_{1}\right)$ reduction of the classes with same name in $\pi_{*}(S)$, of degrees $2 p-3$ and $2 p^{2}-2 p-2$, respectively. The class $\beta_{1}^{\prime}$ is the $\bmod \left(v_{1}\right)$ reduction of the class with same name in $V(0)_{*}$ that supports a primary $\bmod (p)$ homotopy Bockstein $\beta_{0,1}\left(\beta_{1}^{\prime}\right)=\beta_{1}$, and is of degree $2 p^{2}-2 p-1$. The classes $v_{2}$ and $\left(\alpha_{1} \beta_{1}\right)^{\sharp}$, of degree $2 p^{2}-2$ and $2 p^{2}+2 p-6$ respectively, support a primary $\bmod \left(v_{1}\right)$ homotopy Bockstein, given by $\beta_{1,1}\left(v_{2}\right)=\beta_{1}^{\prime}$ and $\beta_{1,1}\left(\left(\alpha_{1} \beta_{1}\right)^{\sharp}\right)=\alpha_{1} \beta_{1}$. The class $v_{2}$ is non-nilpotent. The lowest-degree class in $V(1)_{*}$ that is not in the image of (2.3) is the $\bmod \left(p, v_{1}\right)$ reduction of the class $\beta_{2}$ in $\pi_{*}(S)$, of degree $4 p^{2}-2 p-4$.

If $X$ is a connective spectrum of finite type, the Atiyah-Hirzebruch spectral sequence

$$
E_{s, t}^{2}=H_{s}\left(X ; \mathbb{F}_{p}\right) \otimes V(1)_{t} \Rightarrow V(1)_{s+t} X
$$

converges strongly, and we can use it to compute $V(1)_{*} X$ in low degrees. The first non-trivial Postnikov invariant of $V(1)$ is Steenrod's reduced power operation $P^{1}$, corresponding to the first possibly non-trivial differential of the spectral sequence on the zeroth line, see Remark 2.4. This operation detects the class $\alpha_{1}$, which belongs to the kernel of the Hurewicz homomorphism $V(1)_{*} \rightarrow H_{*}\left(V(1) ; \mathbb{F}_{p}\right)$. In some more details, we have a commutative diagram

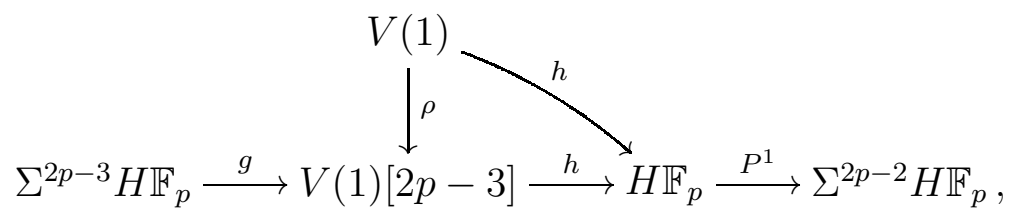

where $\rho$ is the $(2 p-3)$ th-Postnikov section, and the horizontal sequence is a cofibre sequence. Notice that by (2.3) the map $\rho$ is $\left(2 p^{2}-2 p-2\right)$-connected, so that under our assumptions on $X$ we have a well defined homomorphism

$$
\alpha=\left(\rho_{*}\right)^{-1} g_{*}: H_{n-2 p+3}\left(X ; \mathbb{F}_{p}\right) \rightarrow V(1)_{n} X
$$

for $n \leqslant 2 p^{2}-2 p-3$. 
Lemma 2.3. Let $X$ be a connective spectrum of finite type, and let $p \geqslant 3$ be a prime. For $n \leqslant 2 p^{2}-2 p-3$, the group $V(1)_{n} X$ fits in an exact sequence

$$
\begin{aligned}
& H_{n+1}\left(X ; \mathbb{F}_{p}\right) \stackrel{\left(P^{1}\right)^{*}}{\longrightarrow} H_{n-2 p+3}\left(X ; \mathbb{F}_{p}\right) \stackrel{\alpha}{\longrightarrow} V(1)_{n} X \stackrel{h_{*}}{\longrightarrow} \\
& H_{n}\left(X ; \mathbb{F}_{p}\right) \stackrel{\left(P^{1}\right)^{*}}{\longrightarrow} H_{n-2 p+2}\left(X ; \mathbb{F}_{p}\right) .
\end{aligned}
$$

Here $\left(P^{1}\right)^{*}$ denotes the homology operation dual to $P^{1}$. If $X$ is a ring spectrum then $\alpha$ sends the unit $1 \in H_{0}\left(X ; \mathbb{F}_{p}\right)$ to $\alpha_{1}$. Moreover, for any $X$ and any $n \geqslant 0$, we have a commutative diagram

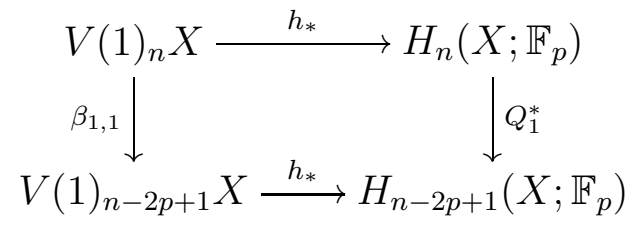

relating the primary mod $\left(v_{1}\right)$ homotopy Bockstein $\beta_{1,1}$ to the homology operation $Q_{1}^{*}$ dual to Milnor's primitive $Q_{1}=P^{1} \delta-\delta P^{1} \in A$.

Proof. This exact sequence is the sequence associated to the cofibre sequence in (2.5), where we have replaced $V(1)[2 p-3]_{n} X$ by $V(1)_{n} X$ via $\rho_{*}$, which is an isomorphism for these values of $n$, by strong convergence of the Atiyah-Hirzebruch spectral sequence. The assertion on $\alpha_{1}$ is true if $X=S$, and follows by naturality for $X$ an arbitrary ring spectrum.

The self-map $f=\left(\Sigma^{2 p-1} i_{1}\right) j_{1}$ of $V(1)$, which induces $\beta_{1,1}$, is given in $\bmod (p)$ homology by the homomorphism $f_{*}: E\left(\tau_{0}, \tau_{1}\right) \rightarrow E\left(\tau_{0}, \tau_{1}\right)$ of degree $1-2 p$ with $f_{*}(1)=f_{*}\left(\tau_{0}\right)=0$, $f_{*}\left(\tau_{1}\right)=1$ and $f_{*}\left(\tau_{0} \tau_{1}\right)=\tau_{0}$. We have a commutative diagram

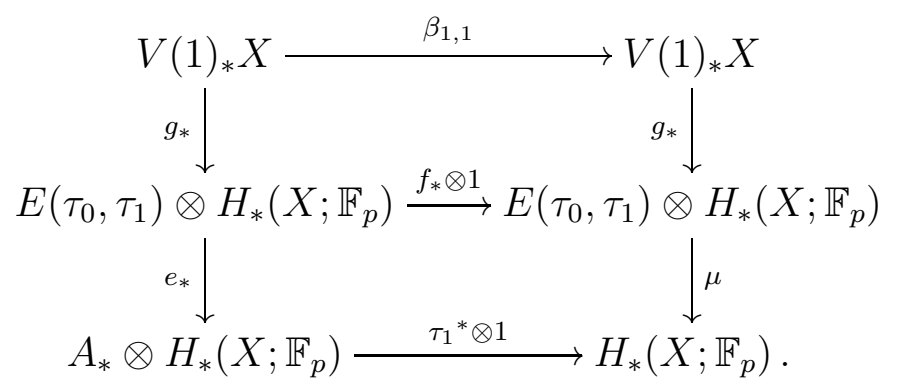

The horizontal arrows are of degree $1-2 p$, and $\tau_{1}{ }^{*}: A_{*} \rightarrow \mathbb{F}_{p}$ is the dual of $\tau_{1}$ with respect to the standard basis $\{\tau(E) \xi(R)\}$ of $A_{*}$ given in [33, $\left.\S 6\right]$. The homomorphism $g_{*}$ is induced in homotopy by the smash product of the unit $S \rightarrow H \mathbb{F}_{p}$ with the identity of $V(1) \wedge X, \mu$ is induced by the right homotopy action $H \mathbb{F}_{p} \wedge V(1) \rightarrow H \mathbb{F}_{p}$, and $e_{*}$ is induced by $1 \wedge h \wedge 1: H \mathbb{F}_{p} \wedge V(1) \wedge X \rightarrow H \mathbb{F}_{p} \wedge H \mathbb{F}_{p} \wedge X$. We have $\mu g_{*}=h_{*}$ and $e_{*} g_{*}=\nu_{*} h_{*}$, where $\nu_{*}$ is the left $A_{*}$-coaction on $H_{*}\left(X ; \mathbb{F}_{p}\right)$. This completes the proof since $\left(\tau_{1}^{*} \otimes 1\right) \nu_{*}=Q_{1}^{*}$ by definition of $Q_{1}$, see [33, page 163].

Remark 2.4. For $X$ connective, the Atiyah-Hirzebruch spectral sequence (2.4) has only two non-trivial lines in internal degrees $t \leqslant 2 p^{2}-2 p-3$, corresponding to 1 and $\alpha_{1}$ in $V(1)_{*}$, see (2.3). The argument above shows that there is a differential

$$
d^{2 p-2}(z)=\left(P^{1}\right)^{*}(z) \alpha_{1}
$$

for $z \in E_{*, 0}^{2}$. In total degrees less than $2 p^{2}-2 p-3$ this is the only possibly non-trivial differential. 
Lemma 2.5. The map

$$
\mathbb{F}_{p}\left\{\alpha_{1}\right\} \oplus P_{p}(x) \rightarrow V(1)_{*} K(\mathbb{Z}, 2)
$$

given by $x \mapsto \sigma \sigma(1)$ with $|x|=2$ is an isomorphism in degrees less than $4 p-3$.

Proof. This follows from Theorem 2.1, Lemma 2.3 and the relation

$$
\left(P^{1}\right)^{*}\left(\gamma_{k+p-1}(y)\right)=k \gamma_{k}(y)
$$

in $H_{*}\left(K(\mathbb{Z}, 2) ; \mathbb{F}_{p}\right) \cong \Gamma(y)$.

Consider the cofibration

$$
B_{1}=\Sigma K(\mathbb{Z}, 2) \stackrel{i}{\longrightarrow} B_{2} \stackrel{j}{\longrightarrow} \Sigma^{2}\left(K(\mathbb{Z}, 2)^{\wedge 2}\right) \rightarrow \Sigma^{2} K(\mathbb{Z}, 2)
$$

extracted from the bar filtration (2.1) of $K(\mathbb{Z}, 3)$. It induces an exact sequence

$$
V(1)_{*} \Sigma K(\mathbb{Z}, 2) \stackrel{i_{*}}{\longrightarrow} V(1)_{*} B_{2} \stackrel{j_{*}}{\longrightarrow} \widetilde{V(1)_{*}} \Sigma^{2}\left(K(\mathbb{Z}, 2)^{\wedge 2}\right) \stackrel{\Sigma^{2} \mu_{*}}{\longrightarrow} V(1)_{*} \Sigma^{2} K(\mathbb{Z}, 2),
$$

where $\mu_{*}$ is induced by the product on $K(\mathbb{Z}, 2)$. We know that $V(1)_{2 p+1} K(\mathbb{Z}, 2)=0$, by Lemma 2.5, which implies that the homomorphism

$$
V(1)_{2 p+2} B_{2} \stackrel{j_{*}}{\longrightarrow} \widetilde{V(1)_{2 p}} K(\mathbb{Z}, 2)^{\wedge 2}
$$

is injective. We know as well that the composition

$$
\widetilde{V(1)_{*}} K(\mathbb{Z}, 2) \otimes \widetilde{V(1)_{*}} K(\mathbb{Z}, 2) \stackrel{k}{\longrightarrow} \widetilde{V(1)_{*}} K(\mathbb{Z}, 2)^{\wedge 2} \stackrel{\mu_{*}}{\longrightarrow} V(1)_{*} K(\mathbb{Z}, 2)
$$

sends the class $x^{p-1} \otimes x$ to zero. In particular, the class $k\left(x^{p-1} \otimes x\right)$ is in the image of $j_{*}$. Let $\tilde{b}^{\prime} \in V(1)_{2 p+2} B_{2}$ be the unique class which satisfies the equation

$$
j_{*}\left(\tilde{b}^{\prime}\right)=\Sigma^{2} k\left(x^{p-1} \otimes x\right) .
$$

Definition 2.6. We define the fundamental class $e_{0}^{\prime} \in V(1)_{3} K(\mathbb{Z}, 3)$ as the image of the unit $1 \in V(1)_{0} K(\mathbb{Z}, 0)$ under the iterated suspension $\sigma^{3}$. We define

$$
b^{\prime} \in V(1)_{2 p+2} K(\mathbb{Z}, 3)
$$

as $b^{\prime}=l_{2 *}\left(\tilde{b}^{\prime}\right)$, where $l_{2}: B_{2} \rightarrow K(\mathbb{Z}, 3)$ is the inclusion of the second subspace in the bar filtration.

Notice that the definition of $b^{\prime}$ in $V(1)$-homotopy, using $x^{p-1} \otimes x$ as above, lifts the definition of the transpotence in the homology of the bar construction. We use this fact in the proof of the following proposition.

Proposition 2.7. The class $b^{\prime} \in V(1)_{*} K(\mathbb{Z}, 3)$ is non-nilpotent, and satisfies the relation

$$
b^{\prime p}=-v_{2} b^{\prime} .
$$

There is a primary mod $\left(v_{1}\right)$ homotopy Bockstein

$$
\beta_{1,1}\left(b^{\prime}\right)=e_{0}^{\prime} .
$$

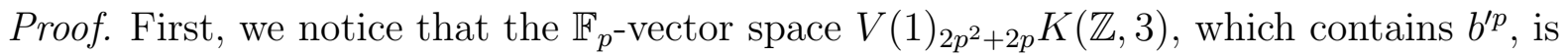
of rank at most one. Indeed, consider the Atiyah-Hirzebruch spectral sequence

$$
E_{s, t}^{2} \cong H_{s}\left(K(\mathbb{Z}, 3) ; \mathbb{F}_{p}\right) \otimes V(1)_{t} \Rightarrow V(1)_{s+t} K(\mathbb{Z}, 3) .
$$

From Theorem 2.1 and the formula (2.3) for $V(1)_{*}$ in low degrees we deduce that $E_{*, *}^{2}$ consists of $\mathbb{F}_{p}\left\{f_{0} \cdot v_{2}, e_{0} \cdot f_{0} \cdot \alpha_{1} \cdot \beta_{1}\right\}$ in total degree $2 p^{2}+2 p$. Suspending the relation (2.6) for $k=1$ we get a relation

$$
\left(P^{1}\right)^{*}\left(e_{1}\right)=e_{0}
$$


Notice that for degree reasons the class $e_{1} \cdot f_{0} \cdot \beta_{1} \in E_{*, *}^{2}$ survives to $E_{*, *}^{2 p-2}$ as a product of $e_{1}$ and $f_{0} \cdot \beta_{1}$. By Remark 2.4, and since $f_{0} \cdot \beta_{1}$ is a cycle, we have a differential

$$
d^{2 p-2}\left(e_{1} \cdot f_{0} \cdot \beta_{1}\right)=e_{0} \cdot f_{0} \cdot \alpha_{1} \cdot \beta_{1},
$$

and this implies the claim on $V(1)_{2 p^{2}+2 p} K(\mathbb{Z}, 3)$.

The unit map $S \rightarrow K(2)$ factors through a map of ring spectra $V(1) \rightarrow K(2)$. The induced ring homomorphism

$$
V(1)_{*} K(\mathbb{Z}, 2) \rightarrow K(2)_{*} K(\mathbb{Z}, 2)
$$

maps $x$ to $\beta_{(0)}$, since these classes are defined as the double suspension of the unit in $V(1)_{0} K(\mathbb{Z}, 0)$, respectively $K(2)_{0} K(\mathbb{Z}, 0)$. By construction, the class $b^{\prime}$ maps to the transpotence of $\beta_{(0)}$, which is $b_{(0,1)}$. We deduce that the sub- $V(1)_{*}$-algebra of $V(1)_{*} K(\mathbb{Z}, 3)$ generated by $b^{\prime}$ maps surjectively onto the subalgebra

$$
P\left(v_{2}, b_{(0,1)}\right) /\left(b_{(0,1)}^{p}+v_{2} b_{(0,1)}\right)
$$

of $K(2)_{*} K(\mathbb{Z}, 3)$ generated by $v_{2}$ and $b_{(0,1)}$. In particular $b^{\prime}$ is non-nilpotent. Thus $V(1)_{2 p^{2}+2 p} K(\mathbb{Z}, 3)$ is of rank one, and injects into $K(2)_{2 p^{2}+2 p} K(\mathbb{Z}, 3)$. This implies the identity $b^{\prime p}=-v_{2} b^{\prime}$.

To prove the Bockstein relation, we map to homology. The Hurewicz homomorphism $h_{*}: V(1)_{*} K(\mathbb{Z}, 3) \rightarrow H_{*}\left(K(\mathbb{Z}, 3) ; \mathbb{F}_{p}\right)$ is an isomorphism in degrees 3 and $2 p+2$, mapping $e_{0}^{\prime}$ to $e_{0}$ and $b^{\prime}$ to the transpotence $\varphi(y)=f_{0}$ of $y$. We have a primary homology Bockstein $\beta\left(f_{0}\right)=e_{1}$ by Theorem 2.1, and combining with (2.7) we obtain $\left(P^{1}\right)^{*} \beta\left(f_{0}\right)=e_{0}$. We also have $\beta\left(P^{1}\right)^{*}\left(f_{0}\right)=0$ for degree reasons. Finally,

$$
Q_{1}^{*}\left(f_{0}\right)=\left(\left(P^{1}\right)^{*} \beta-\beta\left(P^{1}\right)^{*}\right)\left(f_{0}\right)=e_{0},
$$

so by Lemma 2.3 the relation $\beta_{1,1}\left(b^{\prime}\right)=e_{0}^{\prime}$ holds.

\section{The units of $k u$ And the higher Bott ElEment}

The aim of this section is to define low-dimensional classes in $V(1)_{*} K(k u)$ by using the inclusion of units.

We recall from [30] or [31, Definition 7.6] that the space of units $G L_{1}(A)$ of an $E_{\infty}$-ring spectrum $A$ is defined by the following pull-back square of spaces

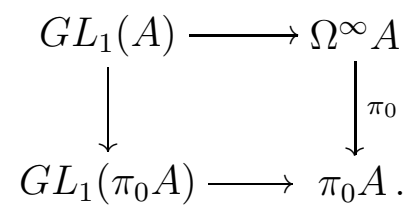

Taking the vertical fiber over $1 \in G L_{1}\left(\pi_{0} A\right)$, we obtain a fiber sequence of group-like $E_{\infty}$-spaces or infinite loop spaces

$$
S L_{1}(A) \rightarrow G L_{1}(A) \rightarrow G L_{1}\left(\pi_{0} A\right),
$$

with products given by the multiplicative structure of $A$. Here we can assume that we have a model of $G L_{1}(A)$ and of $S L_{1}(A)$ which is actually a topological monoid, see for example [39, §2.3]. The functor $G L_{1}$ from $E_{\infty}$-ring spectra to infinite loop spaces is right adjoint, up to homotopy, to the suspension functor $\Sigma_{+}^{\infty}$. This follows from [31, Lemma 9.6]. 
In the case of $k u$, the space $S L_{1}(k u)$ is commonly denoted $B U_{\otimes}$. This notation refers to the product of the underlying $H$-space of $B U_{\otimes}$, which represents the tensor product of virtual line bundles.

The first Postnikov section $\pi: B U_{\otimes} \rightarrow K(\mathbb{Z}, 2)$, with homotopy fiber denoted by $B S U_{\otimes}$, admits a section $j: K(\mathbb{Z}, 2) \simeq B U(1) \rightarrow B U_{\otimes}$. Here the map $j$ represents viewing a line bundle as a virtual line bundle. Both $\pi$ and $j$ are infinite loop maps, and we have a splitting of infinite loop-spaces

$$
B U_{\otimes} \simeq K(\mathbb{Z}, 2) \times B S U_{\otimes},
$$

see [30, V.3.1]. We denote by $B j: K(\mathbb{Z}, 3) \rightarrow B B U_{\otimes}$ a first delooping of $j$, fitting in a homotopy commutative diagram

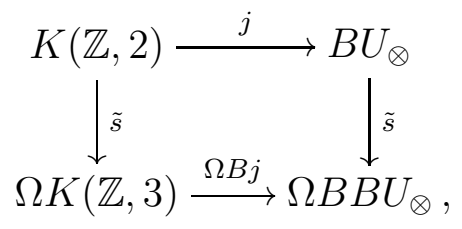

where $\tilde{s}$ denotes the homotopy equivalence which is right adjoint to the suspension $s$ as in $(2.2)$. We name $y_{1} \in \pi_{2} K(\mathbb{Z}, 2) \cong \mathbb{Z}$ the generator that maps to $y \in H_{2}\left(K(\mathbb{Z}, 2) ; \mathbb{F}_{p}\right)$ by the Hurewicz homomorphism. We have maps of based spaces

$$
K(\mathbb{Z}, 2) \stackrel{j}{\longrightarrow} B U_{\otimes} \stackrel{c_{0}}{\longrightarrow} B U \times\{0\} \subset B U \times \mathbb{Z},
$$

where $c_{0}$ is the inclusion in $B U \times \mathbb{Z}$ followed by the translation of the component of 1 to that of 0 in the $H$-group $B U \times \mathbb{Z}$. The map $c_{0} j$ is a $\pi_{2}$-isomorphism, and we define

$$
u=c_{0 *} j_{*}\left(y_{1}\right) \in \pi_{2}(B U \times \mathbb{Z}) .
$$

We call $u$ the Bott class. We have an isomorphism of rings

$$
\pi_{*}(B U \times \mathbb{Z})=\pi_{*} k u \cong \mathbb{Z}[u]
$$

given by Bott periodicity. The map $c_{0_{*}}: \pi_{*}\left(B U_{\otimes}\right) \rightarrow \pi_{*}(B U \times \mathbb{Z})$ is an isomorphism in positive degrees, and we define $y_{n} \in \pi_{2 n}\left(B U_{\otimes}\right)$ by requiring $c_{0 *}\left(y_{n}\right)=u^{n}$. Finally, we define

$$
\sigma_{n}^{\prime} \in V(1)_{2 n+1} B B U_{\otimes}
$$

as the image of $y_{n}$ under the composition

$$
\pi_{2 n} B U_{\otimes} \stackrel{h_{*}}{\longrightarrow} V(1)_{2 n} B U_{\otimes} \stackrel{\sigma}{\longrightarrow} V(1)_{2 n+1} B B U_{\otimes} .
$$

Here the first map is the Hurewicz homomorphism from (unstable) homotopy to $V(1)$ homotopy, and $\sigma$ is the suspension induced by the map $s: \Sigma B U_{\otimes} \rightarrow B B U_{\otimes}$.

Lemma 3.1. Consider the homomorphism

$$
B j_{*}: V(1)_{3} K(\mathbb{Z}, 3) \rightarrow V(1)_{3} B B U_{\otimes}
$$

induced by the map defined above. We have $\sigma_{1}^{\prime}=(B j)_{*}\left(e_{0}^{\prime}\right)$, where $e_{0}^{\prime}=\sigma^{3}(1) \in$ $V(1)_{3} K(\mathbb{Z}, 3)$, as given in Definition 2.6 .

Proof. We have a commutative diagram

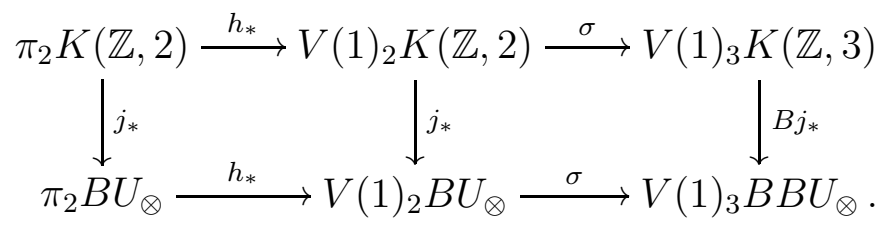


The right-hand square is induced in $V(1)$-homotopy from the square left adjoint to the square (3.1). The class $y_{1} \in \pi_{2} K(\mathbb{Z}, 2)$ was chosen so that $h_{*}\left(y_{1}\right)=\sigma^{2}(1)$ in $V(1)_{2} K(\mathbb{Z}, 2) \cong H_{2}\left(K(\mathbb{Z}, 2) ; \mathbb{F}_{p}\right)$. The lemma follows, since

$$
\sigma_{1}^{\prime}=\sigma h_{*} j_{*}\left(y_{1}\right)=(B j)_{*} \sigma h_{*}\left(y_{1}\right)=(B j)_{*} \sigma^{3}(1)=(B j)_{*}\left(e_{0}^{\prime}\right) .
$$

The space $\Omega^{\infty} K(k u)$ is defined as the group completion of the topological monoid $\coprod_{n} B G L_{n}(k u)$, with product modelling the block-sum of matrices, see for instance [20, VI.7]. The composition

$$
w: B B U_{\otimes} \rightarrow B G L_{1}(k u) \rightarrow \coprod_{n} B G L_{n}(k u) \rightarrow \Omega^{\infty} K(k u)
$$

factors through an infinite loop map $B B U_{\otimes} \rightarrow S L_{1} K(k u)$, which is right adjoint to a map

$$
\omega: \Sigma_{+}^{\infty} B B U_{\otimes} \rightarrow K(k u)
$$

of commutative $S$-algebras. We consider also the map of commutative $S$-algebras

$$
\phi: \Sigma_{+}^{\infty} K(\mathbb{Z}, 3) \rightarrow K(k u)
$$

defined as the composition of the suspension of $B j: K(\mathbb{Z}, 3) \rightarrow B B U_{\otimes}$ with the map $\omega$.

Definition 3.2. For $n \geqslant 1$, we define

$$
\sigma_{n}=\omega_{*}\left(\sigma_{n}^{\prime}\right) \in V(1)_{2 n+1} K(k u),
$$

where $\sigma_{n}^{\prime}$ is the class given in (3.2). We define the "higher Bott element" as

$$
b=\phi_{*}\left(b^{\prime}\right) \in V(1)_{2 p+2} K(k u),
$$

where $b^{\prime} \in V(1)_{2 p+2} K(\mathbb{Z}, 3)$ is the class given in Definition 2.6.

Remark 3.3. Notice that by Proposition 2.7 the classes $b$ and $\sigma_{1}$ are related by a primary $\bmod \left(v_{1}\right)$ homotopy Bockstein $\beta_{1,1}(b)=\sigma_{1}$.

Remark 3.4. Assume that $p$ is an odd prime. If $R$ is a number ring containing a primitive $p$-th root of unity $\zeta_{p}$, for example $R=\mathbb{Z}\left[\zeta_{p}\right]$, then the $\bmod (p)$ algebraic $K$-theory of $R$ contains a non-nilpotent class

$$
\beta \in V(0)_{2} K(R),
$$

called the Bott element, which we referred to in the introduction. It was defined by Browder [15] using the composition

$$
B C_{p} \rightarrow B G L_{1} R \rightarrow \Omega^{\infty} K(R)
$$

analogous to (3.3), and its adjoint

$$
\phi: \Sigma_{+}^{\infty} B C_{p} \rightarrow K(R)
$$

Here $C_{p}$ denotes the cyclic subgroup of order $p$ of $G L_{1}(R)$ generated by $\zeta_{p}$. By inspection, the class $x=\zeta_{p}-1$ satisfies $x^{p}=0$ in the group-ring $\mathbb{F}_{p}\left[C_{p}\right]=V(0)_{0} C_{p}$, and has a well defined "transpotence" $\beta^{\prime} \in V(0)_{2} B C_{p}$, supporting a primary $\bmod (p)$ homotopy Bockstein $\beta_{0,1}\left(\beta^{\prime}\right) \doteq \sigma(1) \in V(0)_{1} B C_{p}$. The classical Bott element can then be defined as

$$
\beta=\phi_{*}\left(\beta^{\prime}\right) \in V(0)_{2} K(R) .
$$

An embedding of rings $R \subset \mathbb{C}^{\text {top }}$, where $\mathbb{C}^{\text {top }}$ has the Euclidean topology, induces a map of commutative $S$-algebras $\iota: K(R) \rightarrow K\left(\mathbb{C}^{\text {top }}\right)=k u$ in algebraic $K$-theory. Browder's 
Proposition [15, 2.2] implies that $\iota_{*} \phi_{*}\left(\beta^{\prime}\right)=u$, where $u$ is the Bott class in $V(0)_{*} k u \cong$ $P(u)$. This proves that $\beta$ is non-nilpotent and is related to the Bott periodicity of topological $K$-theory. Snaith showed [40] that the relation $\beta^{\prime p}=v_{1} \beta^{\prime}$ in $V(0)_{*} B C_{p}$ promotes to the relation

$$
\beta^{p-1}=v_{1}
$$

in $V(0)_{*} K(R)$.

The remark above makes it clear that our construction of $b \in V(1)_{2 p+2} K(k u)$ is inspired from the classical Bott element, and that these classes share interesting properties. This provides some justification for calling $b$ a higher Bott element. Here higher refers to the fact that $b$ lives one chromatic step higher than $\beta$, in the sense that it is defined only in algebraic $K$-theory modulo $\left(p, v_{1}\right)$ and that it is related to $v_{2}$-periodicity. Indeed, recall from Theorem 1.1 and Proposition 1.3 that $b$ is non-nilpotent and that the relation $b^{\prime p}=-v_{2} b^{\prime}$ in $V(1)_{*} K(\mathbb{Z}, 3)$ promotes to the relation

$$
b^{p-1}=-v_{2}
$$

in $V(1)_{*} K(k u)$. Our proof of these assertions relies on the computation of the cyclotomic trace for $k u$, and is much more technical then in the number ring case: unfortunately, in the present situation we don't have an analogue of the map $K(R) \rightarrow K\left(\mathbb{C}^{\text {top }}\right)$, but see the remark below for a possible candidate.

Remark 3.5. John Rognes conjectured [4] that if $\Omega_{1}$ is a separably closed $K(1)$-local pro-Galois extension of $k u$, in the sense of [38], then there is a weak equivalence

$$
L_{K(2)} K\left(\Omega_{1}\right) \simeq E_{2}
$$

where $L_{K(2)}$ is the Bousfield localization functor with respect to the Morava $K$-theory $K(2)$, and where $E_{2}$ is the second Morava $E$-theory spectrum [21] with coefficients

$$
\left(E_{2}\right)_{*}=W\left(\mathbb{F}_{p^{2}}\right)\left[\left[u_{1}\right]\right]\left[u, u^{-1}\right] .
$$

This would provide a map

$$
\iota: K(k u) \rightarrow L_{K(2)} K\left(\Omega_{1}\right) \simeq E_{2}
$$

that might play the role, at this chromatic level, of the map $K(R) \rightarrow K\left(\mathbb{C}^{\text {top }}\right)$ mentioned in Remark [3.4. Since $V(1)_{*} E_{2} \cong \mathbb{F}_{p^{2}}\left[u, u^{-1}\right]$ with $u^{p^{2}-1}=v_{2}$, we presume that the class $b$ would be detected by the non-nilpotent class

$$
\iota_{*}(b)=\alpha u^{p+1} \in V(1)_{*} E_{2}
$$

for some $\alpha \in \mathbb{F}_{p^{2}} \backslash \mathbb{F}_{p}$ with $\alpha^{p-1}=-1$. More generally, we expect that a periodic higher Bott element can be defined in $V(1)_{*} K(A)$ if $A$ is an commutative $S$-algebra with an $S$-algebra map $A \rightarrow \Omega_{1}$ and a suitable $(p-1)$ th-root of $v_{1}$ in $V(0)_{*} A$.

\section{THE TRACE MAP}

In this section, we consider the Bökstedt trace map [11]

$$
\operatorname{tr}: K(k u) \rightarrow T H H(k u)
$$

to topological Hochschild homology. This is a map of commutative $S$-algebras, and it induces a homomorphism of graded-commutative algebras in $V(1)$-homotopy, which we just call the trace. Our aim here is to prove that for $n \leqslant p-2$ the classes $\sigma_{n}$ and $b$ defined above are non-zero in $V(1)_{*} K(k u)$, as well as some of their products, see Proposition 4.6. 
We achieve this by showing that these classes have a non-zero trace in $V(1)_{*} T H H(k u)$. To this end, we briefly recall the computation of $V(1)_{*} T H H(k u)$ given in [1, 9.15].

The topological Hochschild homology spectrum $T H H(k u)$ is a $k u$-algebra, and its $V(1)$ homotopy groups form an algebra over the truncated polynomial algebra $V(1)_{*} k u=$ $P_{p-1}(u)$, where we also denote by $u$ the $\bmod \left(p, v_{1}\right)$ reduction of the Bott class $u \in$ $\pi_{2} k u$. There is a free $\mathbb{F}_{p}$-sub-algebra $E\left(\lambda_{1}\right) \otimes P(\mu)$ in $V(1)_{*} T H H(k u)$, and there is an isomorphism of $E\left(\lambda_{1}\right) \otimes P(\mu) \otimes P_{p-1}(u)$-modules

$$
V(1)_{*} T H H(k u) \cong E\left(\lambda_{1}\right) \otimes P(\mu) \otimes Q_{*},
$$

where $Q_{*}$ is the $P_{p-1}(u)$-module given by

$$
Q_{*}=P_{p-1}(u) \oplus P_{p-2}(u)\left\{a_{0}, b_{1}, a_{1}, b_{2}, \ldots, a_{p-2}, b_{p-1}\right\} \oplus P_{p-1}(u)\left\{a_{p-1}\right\} .
$$

The degree of these generators is given by $\left|\lambda_{1}\right|=2 p-1,|\mu|=2 p^{2},\left|a_{i}\right|=2 p i+3$ and $\left|b_{j}\right|=2 p j+2$. The isomorphism (4.1) is an isomorphism of $P_{p-1}(u)$-algebras if the product on the $P_{p-1}(u)$-module generators of $Q_{*}$ is given by the relations

$$
\begin{cases}b_{i} b_{j}=u b_{i+j} & i+j \leqslant p-1, \\ b_{i} b_{j}=u b_{i+j-p} \mu & i+j \geqslant p, \\ a_{i} b_{j}=u a_{i+j} & i+j \leqslant p-1, \\ a_{i} b_{j}=u a_{i+j-p} \mu & i+j \geqslant p, \\ a_{i} a_{j}=0 & 0 \leqslant i, j \leqslant p-1 .\end{cases}
$$

Here by convention $b_{0}=u$. For example we have a product

$$
\left(u^{k} a_{i}\right)\left(u^{l} b_{j}\right)=u^{p-2} a_{p-1}
$$

if $k+l=p-3$ and $i+j=p-1$.

Remark 4.1. The class $\mu$ is called $\mu_{2}$ in [1], but we adopt here the notation of [3].

The classes $u^{n-1} a_{0} \in V(1)_{2 n+1} T H H(k u)$ for $1 \leqslant n \leqslant p-2$ are constructed as follows. The circle action $S_{+}^{1} \wedge T H H(k u) \rightarrow T H H(k u)$ restricts in the homotopy category to a map $d: \Sigma T H H(k u) \rightarrow T H H(k u)$, which in any homology theory $E_{*}$ induces Connes' operator

$$
d: E_{*} T H H(k u) \rightarrow E_{*+1} T H H(k u) .
$$

We have an $S$-algebra map $l: k u \rightarrow T H H(k u)$ given by the inclusion of zero-simplices. Composing the induced map in $E_{*}$-homology with $d$ yields a suspension homomorphism

$$
d l_{*}: E_{*} k u \rightarrow E_{*+1} T H H(k u),
$$

see [32, 3.2] (it is often denoted $\sigma$ ). For $1 \leqslant n \leqslant p-2$, we define the class $u^{n-1} a_{0}$ as the image

$$
u^{n-1} a_{0}=d l_{*}\left(u^{n}\right)
$$

of $u^{n} \in V(1)_{*} k u$. Mapping to homology, we can show that these classes are non-zero. By Lemma 2.3, the Hurewicz homomorphism

$$
h_{*}: V(1)_{*} T H H(k u) \rightarrow H_{*}\left(T H H(k u) ; \mathbb{F}_{p}\right)
$$

is an isomorphism in degrees $* \leqslant 2 p-3$ (notice that $\alpha_{1}=0$ in $V(1)_{*} T H H(k u)$ since $T H H(k u)$ is a $k u$-algebra). Let $x=h_{*}(u) \in H_{2}\left(k u ; \mathbb{F}_{p}\right)$ be the image of $u \in V(1)_{2} k u$. 
We then have $h_{*}\left(u^{n-1} a_{0}\right)=d l_{*}\left(x^{n}\right)$ in $H_{2 n+1}\left(T H H(k u) ; \mathbb{F}_{p}\right)$, and this class represents the permanent cycle $1 \otimes x^{n} \in E_{1,2 n}^{1}(k u)$ in the Bökstedt spectral sequence

$$
\begin{aligned}
& E_{s, *}^{1}(k u)=H_{*}\left(k u ; \mathbb{F}_{p}\right)^{\otimes(s+1)}, \\
& E_{s, *}^{2}(k u)=H H_{s, *}^{\mathbb{F}_{p}}\left(H_{*}\left(k u ; \mathbb{F}_{p}\right)\right) \Rightarrow H_{s+*}\left(T H H(k u) ; \mathbb{F}_{p}\right) .
\end{aligned}
$$

This proves that the classes $h_{*}\left(u^{n-1} a_{0}\right)$ are non-zero for these values of $n$. We refer to [1, $\S 9]$ for more details.

Lemma 4.2. If $1 \leqslant n \leqslant p-2$, the class $\sigma_{n}^{\prime}$ of (3.2) maps to the class $u^{n-1} a_{0}$ under the composition

$$
V(1)_{*} B B U_{\otimes} \stackrel{\omega_{*}}{\longrightarrow} V(1)_{*} K(k u) \stackrel{\operatorname{tr}_{*}}{\longrightarrow} V(1)_{*} T H H(k u) .
$$

Proof. As mentioned above, $h_{*}: V(1)_{2 n+1} T H H(k u) \rightarrow H_{2 n+1}\left(T H H(k u) ; \mathbb{F}_{p}\right)$ is an isomorphism for $n \leqslant p-2$ and maps $u^{n-1} a_{0}$ to $d l_{*}\left(x^{n}\right)$. Thus, passing to homology and using the definition of $\sigma_{n}^{\prime}$ in (3.2), if suffices to prove that the composition

$$
H_{2 n}\left(B U_{\otimes} ; \mathbb{F}_{p}\right) \stackrel{\sigma}{\longrightarrow} H_{2 n+1}\left(B B U_{\otimes} ; \mathbb{F}_{p}\right) \stackrel{\operatorname{tr}_{*} \omega_{*}}{\longrightarrow} H_{2 n+1}\left(T H H(k u) ; \mathbb{F}_{p}\right)
$$

maps $z_{n}=h_{*}\left(y_{n}\right) \in H_{2 n}\left(B U_{\otimes} ; \mathbb{F}_{p}\right)$ to $d l_{*}\left(x^{n}\right)$. Here we also denoted by $h_{*}$ the Hurewicz homomorphism $\pi_{2 n} B U_{\otimes} \rightarrow H_{2 n}\left(B U_{\otimes} ; \mathbb{F}_{p}\right)$. First, we need some information on the trace map. We will use the following commutative diagram of spaces

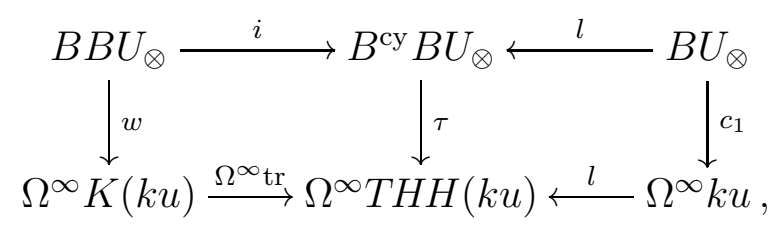

which is assembled from [39, §4]. Here the space $B^{\text {cy }} B U_{\otimes}$ is the realization of the cyclic nerve of the topological monoid $B U_{\otimes}$ and, as $\Omega^{\infty} T H H(k u)$, is equipped with a canonical $S^{1}$-action. The map $\tau$ is the realization of a morphism of cyclic spaces, and is therefore $S^{1}$-equivariant. The maps $l$ are given by the inclusion of 0 -simplices, while $c_{1}$ is the inclusion of the component of 1. There is a homotopy fibration [39, Proposition 3.1]

$$
B U_{\otimes} \stackrel{l}{\longrightarrow} B^{\mathrm{cy}} B U_{\otimes} \stackrel{p}{\longrightarrow} B B U_{\otimes}
$$

and the map $p$ admits a section up to homotopy $i: B B U_{\otimes} \rightarrow B^{\text {cy }} B U_{\otimes}$.

Let $d$ be Connes' operator on $H_{*}\left(B^{\text {cy }} B U_{\otimes} ; \mathbb{F}_{p}\right)$ and $H_{*}\left(\Omega^{\infty} T H H(k u) ; \mathbb{F}_{p}\right)$. It commutes with $\tau_{*}: H_{*}\left(B^{\text {cy }} B U_{\otimes} ; \mathbb{F}_{p}\right) \rightarrow H_{*}\left(\Omega^{\infty} T H H(k u) ; \mathbb{F}_{p}\right)$ since $\tau$ is equivariant. In the next lemma, we prove that

$$
d l_{*}\left(z_{n}\right)=i_{*} \sigma\left(z_{n}\right)
$$

holds in $H_{2 n+1}\left(B^{\mathrm{cy}} B U_{\otimes} ; \mathbb{F}_{p}\right)$. Using (4.4), we deduce

$$
\left(\Omega^{\infty} \operatorname{tr}\right)_{*} w_{*} \sigma\left(z_{n}\right)=\tau_{*} i_{*} \sigma\left(z_{n}\right)=\tau_{*} d l_{*}\left(z_{n}\right)=d \tau_{*} l_{*}\left(z_{n}\right)=d l_{*} c_{1 *}\left(z_{n}\right) .
$$

Finally, composing with the stabilization map

$$
\text { st : } H_{*}\left(\Omega^{\infty} T H H(k u) ; \mathbb{F}_{p}\right) \rightarrow H_{*}\left(T H H(k u) ; \mathbb{F}_{p}\right)
$$

to spectrum homology, we obtain

$$
\operatorname{tr}_{*} \omega_{*} \sigma\left(z_{n}\right)=\operatorname{st}\left(\Omega^{\infty} \operatorname{tr}\right)_{*} w_{*} \sigma\left(z_{n}\right)=\operatorname{st} d l_{*} c_{1 *}\left(z_{n}\right)=d l_{*}\left(x^{n}\right) .
$$

For the last equality, we used that stabilization commutes with $d l_{*}$, and that $\operatorname{stc}_{1 *}\left(z_{n}\right)=$ $x^{n}$ for $1 \leqslant n \leqslant p-2$. 
Lemma 4.3. The equality $d l_{*}\left(z_{n}\right)=i_{*} \sigma\left(z_{n}\right)$ holds in $H_{2 n+1}\left(B^{\mathrm{cy}} B U_{\otimes} ; \mathbb{F}_{p}\right)$.

Proof. We consider the homotopy fibration (4.5). Since $H_{*}\left(B U_{\otimes} ; \mathbb{F}_{p}\right)$ is concentrated in even degrees, the map $p_{*}: H_{*}\left(B^{\mathrm{cy}} B U_{\otimes} ; \mathbb{F}_{p}\right) \rightarrow H_{*}\left(B B U_{\otimes} ; \mathbb{F}_{p}\right)$ restricts to an isomorphism

$$
p_{*}: \operatorname{Prim}\left(H_{2 n+1}\left(B^{\mathrm{cy}} B U_{\otimes} ; \mathbb{F}_{p}\right)\right) \rightarrow \operatorname{Prim}\left(H_{2 n+1}\left(B B U_{\otimes} ; \mathbb{F}_{p}\right)\right)
$$

of the subgroups of primitive elements in degree $2 n+1$, with the restriction of $i_{*}$ as inverse. The class $l_{*}\left(z_{n}\right)$ is spherical, hence primitive, and it follows from $d(1)=0$ that $d l_{*}\left(z_{n}\right)$ is also primitive.

Next, we consider the diagram

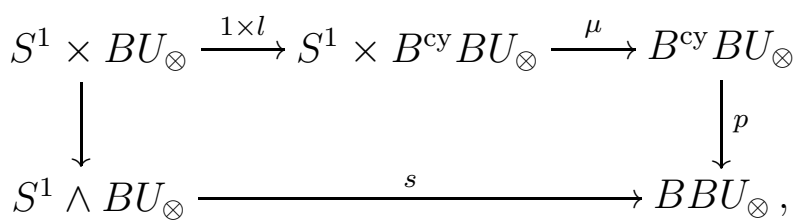

where $\mu$ denotes the $S^{1}$-action on $B^{\text {cy }} B U_{\otimes}$ and $s$ the suspension map (2.2). This diagram is commutative, as can be checked at simplicial level by using the definition of $\mu$, see for example [27, 7.1.9]. Therefore $p_{*} d l_{*}\left(z_{n}\right)=\sigma\left(z_{n}\right)$, and since $d l_{*}\left(z_{n}\right)$ is primitive, we have

$$
d l_{*}\left(z_{n}\right)=i_{*} p_{*} d l_{*}\left(z_{n}\right)=i_{*} \sigma\left(z_{n}\right) .
$$

Lemma 4.4. The class $b^{\prime}$ maps to the class $b_{1}$ under the composition

$$
V(1)_{*} K(\mathbb{Z}, 3) \stackrel{\phi_{*}}{\longrightarrow} V(1)_{*} K(k u) \stackrel{\mathrm{tr}_{*}}{\longrightarrow} V(1)_{*} T H H(k u) .
$$

Proof. We know from Lemma 3.1 and Lemma 4.2 that $e_{0}^{\prime} \in V(1)_{3} K(\mathbb{Z}, 3)$ maps to the class $a_{0}$ in $V(1)_{3} T H H(k u)$. We have primary mod $\left(v_{1}\right)$ homotopy Bockstein

$$
\beta_{1,1}\left(b^{\prime}\right)=e_{0}^{\prime} \text { and } \beta_{1,1}\left(b_{1}\right)=a_{0}
$$

in $V(1)_{*} K(\mathbb{Z}, 3)$ and $V(1)_{*} T H H(k u)$ respectively, see Proposition 2.7 and [1, 9.19]. Moreover $V(1)_{2 p+2} T H H(k u)=\mathbb{F}_{p}\left\{b_{1}\right\}$, so that $\beta_{1,1}$ is injective on this group. The result follows, since

$$
\beta_{1,1} \operatorname{tr}_{*} \phi_{*}\left(b^{\prime}\right)=\operatorname{tr}_{*} \phi_{*} \beta_{1,1}\left(b^{\prime}\right)=\operatorname{tr}_{*} \phi_{*}\left(e_{0}^{\prime}\right)=a_{0}
$$

Let $\kappa: k u \rightarrow k u_{p}$ be the completion at $p$. It induces the inclusion $\mathbb{Z}[u] \rightarrow \mathbb{Z}_{p}[u]$ of coefficients rings.

Definition 4.5. We also denote by

$$
\sigma_{n} \in V(1)_{2 n+1} K\left(k u_{p}\right) \text { and } b \in V(1)_{2 p+2} K\left(k u_{p}\right)
$$

the image under $\kappa_{*}: V(1)_{*} K(k u) \rightarrow V(1)_{*} K\left(k u_{p}\right)$ of the classes $\sigma_{n}$ and $b$ defined in 3.2 ,

Proposition 4.6. The classes

$$
\begin{cases}b^{k} & \text { for } 0 \leqslant k \leqslant p-2, \text { and } \\ \sigma_{n} b^{l} & \text { for } 1 \leqslant n \leqslant p-2 \text { and } 0 \leqslant l \leqslant p-2-n\end{cases}
$$

are non-zero in $V(1)_{*} K(k u)$ and in $V(1)_{*} K\left(k u_{p}\right)$. 
Proof. For $V(1)_{*} K(k u)$, it follows from Lemma 4.2, Lemma 4.4 and the structure of $V(1)_{*} T H H(k u)$ given in (4.2). In more detail, we have $\operatorname{tr}_{*}\left(b^{k}\right)=b_{1}^{k} \neq 0$ for $k \leqslant p-2$ and $\operatorname{tr}_{*}\left(\sigma_{n} b^{l}\right)=u^{n-1} a_{0} b_{1}^{l}=u^{n+l-1} a_{l} \neq 0$ for $l \leqslant p-3$ and $n+l-1 \leqslant p-3$. Notice that we have a commutative diagram

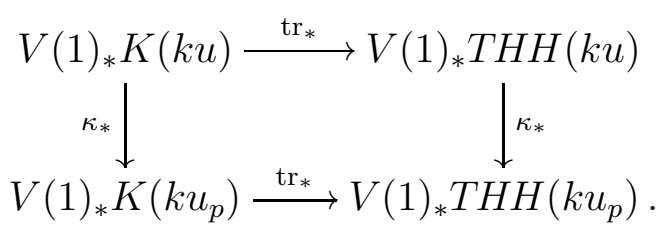

The map $\kappa: T H H(k u) \rightarrow T H H\left(k u_{p}\right)$ is a weak equivalence after $p$-completion, so in this diagram the right-hand $\kappa_{*}$ is an isomorphism. This proves that the result also holds for $V(1)_{*} K\left(k u_{p}\right)$.

Remark 4.7. We claimed in Theorem 1.1 and Proposition 1.3 that $b$ is non-nilpotent in $V(1)_{*} K(k u)$. However, we have

$$
\operatorname{tr}_{*}\left(b^{p-1}\right)=\operatorname{tr}_{*}(b)^{p-1}=b_{1}^{p-1}=u^{p-2} b_{p-1}=0
$$

in $V(1)_{*} T H H(k u)$, so that the Bökstedt trace is not sufficient for proving this assertion. This is of course also predicted by our other claim that $b^{p-1}=-v_{2}$ holds in $V(1)_{*} K(k u)$. Indeed, $v_{2}$ maps to zero in $V(1)_{*} T H H(k u)$ since $T H H(k u)$ is a $k u$-algebra.

\section{Algebraic $K$-Theory in LOW Degrees}

In this section, we compute the groups $V(1)_{*} K\left(k u_{p}\right)$ in degrees $* \leqslant 2 p-2$. This complements the computations presented in the next sections, which are based on evaluating the fixed points of $T H H(k u)$ and which are valid only in degrees larger than $2 p-2$, see Proposition 6.7.

Consider the Adams summand

$$
\ell_{p}=k u_{p}^{h \Delta}
$$

of $k u_{p}$, where $\Delta \cong \mathbb{Z} /(p-1)$ is the finite subgroup of the $p$-adic units, acting on $k u_{p}$ by $p$-adic Adams operations, and where $(-)^{h \Delta}$ denotes the homotopy fixed points. By Theorem 10.2 of [1], the natural map $V(1)_{*} K\left(\ell_{p}\right) \rightarrow V(1)_{*} K\left(k u_{p}\right)$ factors through an isomorphism

$$
V(1)_{*} K\left(\ell_{p}\right) \cong\left(V(1)_{*} K\left(k u_{p}\right)\right)^{\Delta} \subset V(1)_{*} K\left(k u_{p}\right)
$$

onto the elements of $V(1)_{*} K\left(k u_{p}\right)$ fixed under the induced action of $\Delta$. In the sequel, we identify $V(1)_{*} K\left(\ell_{p}\right)$ with its image in $V(1)_{*} K\left(k u_{p}\right)$.

The $V(1)$-homotopy of $K\left(\ell_{p}\right)$ is computed in [3]. In the degrees we are concerned with here, namely $* \leqslant 2 p-2, V(1)_{*} K\left(\ell_{p}\right)$ is generated as an $\mathbb{F}_{p}$-vector space by the classes listed in

$$
\left\{1, \lambda_{1} t^{d}, s, \partial \lambda_{1} \mid 0<d<p\right\} \text {, }
$$

of degree $\left|\lambda_{1} t^{d}\right|=2 p-2 d-1,|s|=2 p-3$ and $\left|\partial \lambda_{1}\right|=2 p-2$, see [3, 9.1] (where the sporadic $v_{2}$-torsion class $s$ was denoted $a$ ). The zeroth Postnikov section $\ell_{p} \rightarrow H \mathbb{Z}_{p}$ is a $(2 p-2)$-connected map, so that the induced map $K\left(\ell_{p}\right) \rightarrow K\left(\mathbb{Z}_{p}\right)$ is $(2 p-1)$ connected [12, Proposition 10.9]. All the classes listed in (5.2) map to classes with same name in $V(1)_{*} K\left(\mathbb{Z}_{p}\right)$, which is given by the formula

$$
V(1)_{*} K\left(\mathbb{Z}_{p}\right) \cong E\left(\lambda_{1}\right) \oplus \mathbb{F}_{p}\left\{s, \partial \lambda_{1}\right\} \oplus \mathbb{F}_{p}\left\{\lambda_{1} t^{d} \mid 0<d<p\right\} .
$$


The name of the classes in this formula refers to permanent cycles in the $S^{1}$ homotopy fixed-point spectral sequence used in the computation of $V(1)_{*} K\left(\mathbb{Z}_{p}\right)$ by traces, compare with Theorem 7.9. If desired, these classes could be given a more memorable name by means of the inclusion

$$
V(0)_{*} K\left(\mathbb{Z}_{p}\right) \rightarrow V(0)_{*} K\left(\mathbb{Q}_{p}\left(\zeta_{p}\right)\right)
$$

in the target of which they can be decomposed as a product of a unit and a power of the Bott element $\beta \in V(0)_{2} K\left(\mathbb{Q}_{p}\left(\zeta_{p}\right)\right)$.

Using the inclusion given in (5.1), we view the classes listed in (5.2) as elements of $V(1)_{*} K\left(k u_{p}\right)$. The following lemma implies that these classes are linearly independent of the classes in $V(1)_{*} K\left(k u_{p}\right)$ constructed in the previous section.

Lemma 5.1. The non-zero classes $b^{k}$ and $\sigma_{n} b^{l}$ in $V(1)_{*} K\left(k u_{p}\right)$ given in Proposition 4.6 are not fixed under the action of $\Delta$.

Proof. All these classes map into $V(1)_{*} T H H(k u)$ to classes which do not lie in the image of $V(1)_{*} T H H\left(\ell_{p}\right)$, and hence which are not fixed under the action of $\Delta$, see Proposition 10.1 of [1].

Proposition 5.2. The inclusion

$$
\mathbb{F}_{p}\left\{1, \sigma_{n}, \lambda_{1} t^{d}, s, \partial \lambda_{1} \mid 1 \leqslant n \leqslant p-2,0<d<p\right\} \subset V(1)_{*} K\left(k u_{p}\right)
$$

of graded $\mathbb{F}_{p}$-vector spaces is an isomorphism in degrees $\leqslant 2 p-2$.

Proof. We have constructed all the classes listed above and have argued that they are linearly independent. It suffices therefore to compute the dimension of $V(1)_{n} K\left(k u_{p}\right)$ as an $\mathbb{F}_{p}$-vector space for all $0 \leqslant n \leqslant 2 p-2$.

Consider a double loop map $\Omega S^{3} \rightarrow B U_{\otimes}$ such that the composition

$$
S^{2} \rightarrow \Omega S^{3} \rightarrow B U_{\otimes}
$$

where $S^{2} \rightarrow \Omega S^{3}$ is the adjunction unit, represents the class $y_{1} \in \pi_{2} B U_{\otimes}$ defined in Section 3. By adjunction we have a map of $E_{2}$-ring spectra

$$
S\left[\Omega S^{3}\right] \rightarrow k u,
$$

where $S\left[\Omega S^{3}\right]$ is another notation for the suspension spectrum $\Sigma_{+}^{\infty} \Omega S^{3}$. We refer to $[5$, Proposition 2.2] for some more details on the construction of this map. After $p$-completion this map is $(2 p-3)$-connected, and induces a $(2 p-2)$-connected map $K\left(S\left[\Omega S^{3}\right]_{p}\right) \rightarrow$

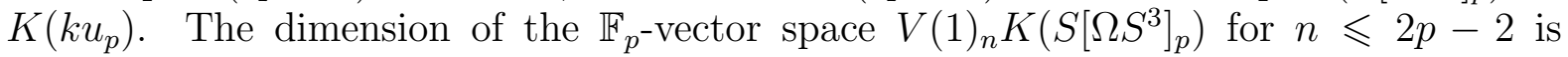
computed in the following lemma, and this completes the proof of this proposition. Notice that a priory

$$
V(1)_{2 p-2} K\left(S\left[\Omega S^{3}\right]_{p}\right) \rightarrow V(1)_{2 p-2} K\left(k u_{p}\right)
$$

is only surjective, but luckily $V(1)_{2 p-2} K\left(S\left[\Omega S^{3}\right]_{p}\right)$ is of rank one. Since we know that the rank of $V(1)_{2 p-2} K\left(k u_{p}\right)$ is at least one, we also have an isomorphism in this degree.

Lemma 5.3. The dimension of $V(1)_{n} K\left(S\left[\Omega S^{3}\right]_{p}\right)$ as an $\mathbb{F}_{p}$-vector space is

$$
\begin{cases}1 & \text { if } n=0,1,2 p-2, \\ 2 & \text { if } n \text { is odd with } 3 \leqslant n \leqslant 2 p-5 \\ 3 & \text { if } n=2 p-3 \\ 0 & \text { for other values of } n \leqslant 2 p-2 .\end{cases}
$$


Proof. We compute $V(1)_{*} K\left(S\left[\Omega S^{3}\right]_{p}\right)$ in degrees less than $2 p-1$ by using the cyclotomic trace map to topological cyclic homology [11], which sits in a cofibre sequence [22]

$$
K\left(S\left[\Omega S^{3}\right]_{p}\right)_{p} \stackrel{\operatorname{trc}}{\longrightarrow} T C\left(S\left[\Omega S^{3}\right]_{p}\right) \rightarrow \Sigma^{-1} H \mathbb{Z}_{p} \rightarrow \Sigma K\left(S\left[\Omega S^{3}\right]_{p}\right)_{p} .
$$

Here $T C(X)=T C(X ; p)$ denotes the ( $p$-completed) topological cyclic homology spectrum of a spectrum $X$. By inspection, it suffices to prove that we have

$$
\operatorname{dim}_{\mathbb{F}_{p}} V(1)_{n} T C\left(S\left[\Omega S^{3}\right]_{p}\right)= \begin{cases}1 & \text { if } n=-1,0,1,2 p-2, \\ 2 & \text { if } n \text { is odd with } 3 \leqslant n \leqslant 2 p-3, \\ 0 & \text { for other values of } n \leqslant 2 p-2 .\end{cases}
$$

Indeed, $V(1)_{*} \Sigma^{-1} H \mathbb{Z}_{p}$ consists of a copy of $\mathbb{F}_{p}$ in degrees -1 and $2 p-2$, and is zero in other degrees. We have an isomorphism $V(1)_{-1} T C\left(S\left[\Omega S^{3}\right]_{p}\right) \rightarrow V(1)_{-1} \Sigma^{-1} H \mathbb{Z}_{p}$, and the sporadic class $s$ is in the image of the connecting homomorphism

$$
V(1)_{2 p-2} \Sigma^{-1} H \mathbb{Z}_{p} \rightarrow V(1)_{2 p-3} K\left(S\left[\Omega S^{3}\right]_{p}\right),
$$

by naturality with respect to $S\left[\Omega S^{3}\right]_{p} \rightarrow H \mathbb{Z}_{p}$, see for example [3, Proof of 9.1].

The reduced topological cyclic homology spectrum $\widetilde{T C}\left(S\left[\Omega S^{3}\right]_{p}\right)$ is the homotopy fibre of the map $c: T C\left(S\left[\Omega S^{3}\right]_{p}\right) \rightarrow T C\left(S_{p}\right)$ induced by the map $S^{3} \rightarrow *$ to a one-point space. The maps $c$ admits a splitting, and we have a decomposition

$$
T C\left(S\left[\Omega S^{3}\right]_{p}\right) \simeq T C\left(S_{p}\right) \vee \widetilde{T C}\left(S\left[\Omega S^{3}\right]_{p}\right) .
$$

The spectrum $T C\left(S_{p}\right)$ decomposes as

$$
T C\left(S_{p}\right) \simeq S_{p} \vee \Sigma \mathbb{C} P_{-1}^{\infty},
$$

where $\mathbb{C} P_{-1}^{\infty}$ is the ( $p$-completed) Thom spectrum of minus the canonical line bundle on $\mathbb{C} P^{\infty}$, see [29]. The homology of $\Sigma \mathbb{C} P_{-1}^{\infty}$ is given by

$$
H_{*}\left(\Sigma \mathbb{C} P_{-1}^{\infty} ; \mathbb{F}_{p}\right) \cong \mathbb{F}_{p}\left\{x_{i} \mid i \geqslant-1\right\}
$$

with $\left|x_{i}\right|=2 i+1$. Moreover these classes can be chosen so that the relations

$$
\left(P^{1}\right)^{*}\left(x_{p-2}\right)=x_{-1} \text { and }\left(P^{1}\right)^{*}\left(x_{p-1}\right)=0
$$

hold. It follows from Lemma 2.3 that we have an inclusion

$$
\mathbb{F}_{p}\left\{c_{i} \mid-1 \leqslant i \leqslant p-3\right\} \cup \mathbb{F}_{p}\left\{\alpha\left(x_{0}\right)\right\} \subset V(1)_{*}\left(\Sigma \mathbb{C} P_{-1}^{\infty}\right),
$$

which is an isomorphism in degrees $* \leqslant 2 p-2$, with $h_{*}\left(c_{i}\right)=x_{i}$. These classes have degree $\left|c_{i}\right|=2 i+1$ and $\left|\alpha\left(x_{0}\right)\right|=2 p-2$.

By [10, 3.9], we have a decomposition

$$
\widetilde{T C}\left(S\left[\Omega S^{3}\right]_{p}\right) \simeq \Sigma^{\infty} S_{p}^{3} \vee \widetilde{V},
$$

where $\widetilde{V}$ is the (p-completed) homotopy fiber of the composition

$$
\Sigma^{\infty} \Sigma\left(E S_{+}^{1} \wedge_{S^{1}} L S^{3}\right) \stackrel{\operatorname{trf}}{\longrightarrow} \Sigma^{\infty} L S^{3} \stackrel{\epsilon_{1}}{\longrightarrow} \Sigma^{\infty} S^{3} .
$$

Here trf is the dimension-shifting $S^{1}$-transfer on the free loop space $L S^{3}$ of $S^{3}$, and $\epsilon_{1}$ is the evaluation at $1 \in S^{1}$, see [29]. We consider the Serre spectral sequence

$$
E_{* *}^{2}=H_{*}\left(B S^{1} ; H_{*}\left(L S^{3}, \mathbb{F}_{p}\right)\right) \Rightarrow H_{*}\left(E S^{1} \times_{S^{1}} L S^{3} ; \mathbb{F}_{p}\right) .
$$


We have isomorphisms

$$
\begin{aligned}
& H_{*}\left(B S^{1} ; \mathbb{F}_{p}\right) \cong H_{*}\left(K(\mathbb{Z}, 2) ; \mathbb{F}_{p}\right)=\Gamma(y) \text { and } \\
& H_{*}\left(L S^{3} ; \mathbb{F}_{p}\right) \cong P(z) \otimes E(d z) .
\end{aligned}
$$

Here $z \in H_{2}\left(\Omega S^{3} ; \mathbb{F}_{p}\right) \subset H_{2}\left(L S^{3} ; \mathbb{F}_{p}\right)$ and $d z \in H_{3}\left(L S^{3} ; \mathbb{F}_{p}\right)$ is the suspension of $z$ associated to the circle action on $L S^{3}$. In particular, we have a non-zero $d^{2}$-differential

$$
d^{2}(y z)=d z .
$$

For degree reasons no further non-zero differential involves the classes in total degree less than $2 p$, and we have an inclusion

$$
P_{p}(y) \oplus \mathbb{F}_{p}\left\{z^{j} \mid 1 \leqslant j \leqslant p-1\right\} \subset H_{*}\left(E S^{1} \times_{S^{1}} L S^{3} ; \mathbb{F}_{p}\right)
$$

which is an isomorphism in degrees less than $2 p$. We deduce that the inclusion

$$
\Sigma \mathbb{F}_{p}\left\{z^{j} \mid 1 \leqslant j \leqslant p-1\right\} \subset H_{*}\left(\Sigma^{\infty} \Sigma\left(E S_{+}^{1} \wedge_{S^{1}} L S^{3}\right) ; \mathbb{F}_{p}\right)
$$

is an isomorphism in degrees less the $2 p-1$. The homomorphism

$$
\left(\epsilon_{1} \operatorname{trf}\right)_{*}: H_{*}\left(\Sigma^{\infty} \Sigma\left(E S_{+}^{1} \wedge_{S^{1}} L S^{3}\right) ; \mathbb{F}_{p}\right) \rightarrow H_{*}\left(S^{3} ; \mathbb{F}_{p}\right)=E(e)
$$

maps $\Sigma z$ to a generator $e$ of $H_{3}\left(S^{3} ; \mathbb{F}_{p}\right)$ since the restriction of $\operatorname{trf}$ to $\Sigma^{\infty} \Sigma\left(S_{+}^{1} \wedge_{S^{1}} L S^{3}\right)$ is induced by the circle action. This implies that we have an inclusion

$$
\mathbb{F}_{p}\left\{e, \Sigma z^{j} \mid 2 \leqslant j \leqslant p-2\right\} \subset H_{*}\left(\Sigma^{\infty} S_{p}^{3} \vee \widetilde{V} ; \mathbb{F}_{p}\right) \cong H_{*}\left(\widetilde{T C}\left(S\left[\Omega S^{3}\right]_{p}\right) ; \mathbb{F}_{p}\right)
$$

which is an isomorphism in degrees smaller than $2 p-1$. By Lemma 2.3

$$
\mathbb{F}_{p}\left\{e, \Sigma z^{j} \mid 2 \leqslant j \leqslant p-2\right\} \subset V(1)_{*} \widetilde{T C}\left(S\left[\Omega S^{3}\right]_{p}\right)
$$

is also an isomorphism in degrees less than $2 p-1$. In summary, we have

$$
V(1)_{*} T C\left(S\left[\Omega S^{3}\right]_{p}\right) \cong V(1)_{*} \oplus V(1)_{*} \Sigma \mathbb{C} P_{-1}^{\infty} \oplus V(1)_{*} \widetilde{T C}\left(S\left[\Omega S^{3}\right]_{p}\right)
$$

which is isomorphic to

$$
\mathbb{F}_{p}\left\{1, \alpha_{1}, c_{i}, \alpha\left(x_{0}\right), e, \Sigma z^{j} \mid-1 \leqslant i \leqslant p-3,2 \leqslant j \leqslant p-2\right\}
$$

in degrees smaller than $2 p-1$. This proves that formula (5.3) for the rank of the $\mathbb{F}_{p}$-vector space $V(1)_{*} T C\left(S\left[\Omega S^{3}\right]_{p}\right)$ is correct.

Remark 5.4. In an earlier proof of this lemma we used the space $B U(1)$ and the map $\theta: \Sigma_{+}^{\infty} B U(1) \rightarrow k u$ of commutative $S$-algebras. I thank John Rognes for noticing that using $\Omega S^{3}$ instead simplifies the computation. The maps

$$
S\left[\Omega S^{3}\right] \rightarrow \Sigma_{+}^{\infty} B U(1) \rightarrow k u
$$

are $\pi_{0}$-isomorphisms and rational equivalences. We use this in [5] to determine the rational homotopy type of $K(k u)$. 


\section{THE FIXED POINTS}

In this section we compute the $V(1)$-homotopy groups of the homotopy limit

$$
T F\left(k u_{p}\right)=\underset{n, F}{\operatorname{holim}} \operatorname{THH}\left(k u_{p}\right)^{C_{p^{n}}},
$$

where $F: T H H\left(k u_{p}\right)^{C_{p^{n+1}}} \rightarrow T H H\left(k u_{p}\right)^{C_{p^{n}}}$ is the Frobenius map. This will be used in the next section to compute the topological cyclic homology of $k u_{p}$. The strategy to perform such computations was developed in [12, 22, 43], but we will closely follow the exposition and adopt the notations of [3, $\S 3, \S 5$ and $\S 6]$, with an exception: the $G$ Tate construction on an equivariant $G$ spectrum $X$ will be denoted by $X^{t G}$ instead of $\hat{\mathbb{H}}(G, X)$. We refer the reader to [3, $\S 3]$ for a brief review of the homotopy commutative norm-restriction diagram

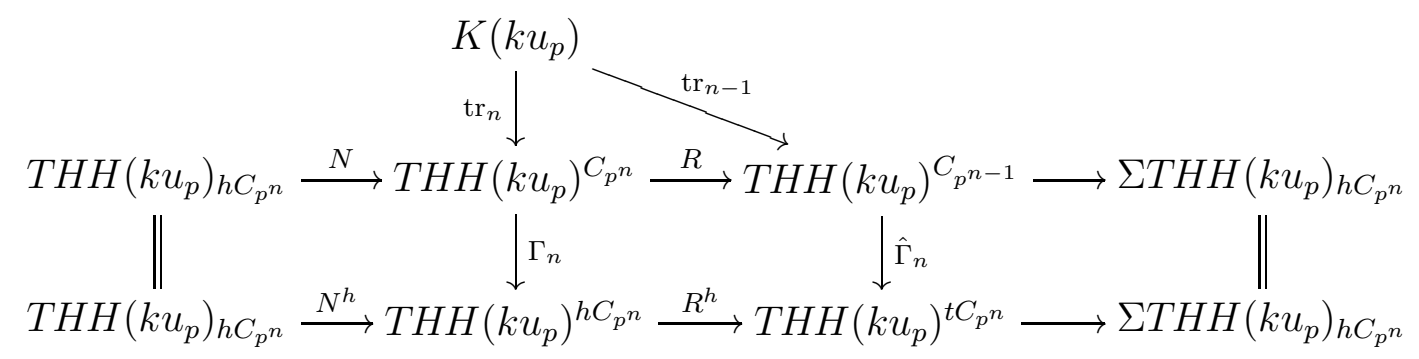

for any $n \geqslant 1$, which is our essential tool. By passage to homotopy limits over the Frobenius maps, we obtain the homotopy commutative diagram

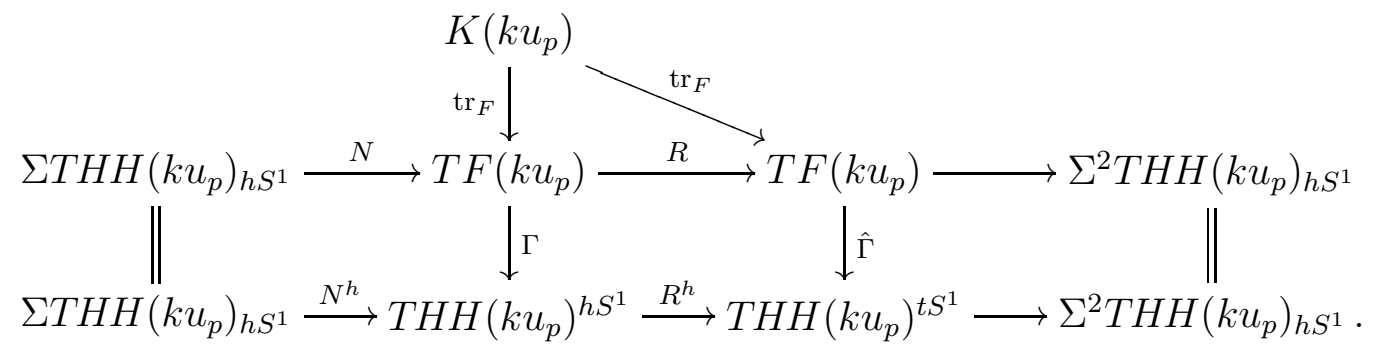

The map $i_{*}: V(1)_{*} T H H\left(\ell_{p}\right) \rightarrow T H H\left(k u_{p}\right)$ factors through an isomorphism onto the $\Delta$-fixed elements of $V(1)_{*} T H H\left(k u_{p}\right)$,

$$
i_{*}: V(1)_{*} T H H\left(\ell_{p}\right) \stackrel{\cong}{\longrightarrow}\left(V(1)_{*} T H H\left(k u_{p}\right)\right)^{\Delta} \subset V(1)_{*} T H H\left(k u_{p}\right),
$$

see [1, 10.1]. The corresponding results hold also for the $C_{p^{n}}$ or $S^{1}$ homotopy fixed points of $T H H$, for the $C_{p^{n}}$ or $S^{1}$ Tate construction on $T H H$, and for $T C$ and $K$, see [1, 10.2]. In the sequel, we identify $V(1)_{*} T H H\left(\ell_{p}\right), V(1)_{*} T C\left(\ell_{p}\right)$, etc. with their image under $i_{*}$. We have a similar statement for the various spectral sequences computing the $V(1)$-homotopy of these spectra.

Lemma 6.1. Let $G=S^{1}$ or $G=C_{p^{n}}$, and let $E^{*}\left(G, \ell_{p}\right)$ and $E^{*}\left(G, k u_{p}\right)$ be the $G$ homotopy fixed-point spectral sequences converging strongly to $V(1)_{*} T H H\left(\ell_{p}\right)^{h G}$ and to $V(1)_{*} T H H\left(k u_{p}\right)^{h G}$, respectively. Then the morphism of spectral sequences induced by the map $\ell_{p} \rightarrow k u_{p}$ is equal to the inclusion of the $\Delta$ fixed points

$$
E^{*}\left(G, \ell_{p}\right)=\left(E^{*}\left(G, k u_{p}\right)\right)^{\Delta} \subset E^{*}\left(G, k u_{p}\right) .
$$


This holds also for the morphism induced on the $G$ Tate spectral sequences converging to $V(1)_{*} T H H\left(\ell_{p}\right)^{t G}$ and $V(1)_{*} T H H\left(k u_{p}\right)^{t G}$, which is given by

$$
\hat{E}^{*}\left(G, \ell_{p}\right)=\left(\hat{E}^{*}\left(G, k u_{p}\right)\right)^{\Delta} \subset \hat{E}^{*}\left(G, k u_{p}\right) .
$$

Proof. The group $\Delta$ acts on $k u_{p}$ by $S$-algebra maps, and it acts $S^{1}$-equivariently on $T H H\left(k u_{p}\right)$. In particular $\Delta$ acts by morphisms of spectral sequences on $E^{*}\left(G, k u_{p}\right)$ and $\hat{E}^{*}\left(G, k u_{p}\right)$, and hence it suffices to prove that the claims hold at the level of the $E^{2}$-terms. This follows from (6.1).

From now on, we will omit $k u_{p}$ from the notation and just write $E^{*}(G)$ and $\hat{E}^{*}(G)$ for the $G$ homotopy fixed-point and Tate spectral sequences converging to $V(1)_{*} T H H\left(k u_{p}\right)^{h G}$ and $V(1)_{*} T H H\left(k u_{p}\right)^{t G}$, respectively.

At this point, we recall the notion of $\delta$-weight introduced in [1, 8.2]. We fix a generator $\delta$ of the group $\Delta$ acting on $k u_{p}, K\left(k u_{p}\right), T H H\left(k u_{p}\right), T C\left(k u_{p}\right)$, etc. The self-map $\delta_{*}$ of $V(1)_{*} k u_{p}=P_{p-1}(u)$ maps $u$ to $\alpha u$ for some generator $\alpha$ of $\mathbb{F}_{p}^{\times}$. We say that a class $v \in V(1)_{*} K\left(k u_{p}\right)$ has $\delta$-weight $i \in \mathbb{Z} /(p-1)$ if $\delta_{*}(v)=\alpha^{i} v$. The same convention holds for classes in $V(1)_{*} T H H\left(k u_{p}\right), V(1)_{*} T C\left(k u_{p}\right)$, etc. For example, the generators $a_{i}$ and $b_{j}$ of $V(1)_{*} T H H\left(k u_{p}\right)$ given in (4.1) all have $\delta$-weight 1, see [1, 10.1]. Similarly, it follows from its definition that $b \in V(1)_{*} K\left(k u_{p}\right)$ has $\delta$-weight 1 . Since $\delta_{*}$ is diagonalizable, we can reinterpret Lemma 6.1 by saying that each of these spectral sequences for $k u_{p}$ has an extra $\mathbb{Z} /(p-1)$-grading given by the $\delta$-weight, and that its homogeneous summand of $\delta$ weight 0 consists of the corresponding spectral sequence for $\ell_{p}$. Together with the internal and filtration degrees, the $\delta$-weight endows the $E^{r}$-terms of these spectral sequences with a tri-grading that we will refer to in the computations below.

By a computation of McClure and Staffeldt [32, [3, 2.6], we have an isomorphism of $\mathbb{F}_{p}$-algebras

$$
V(1)_{*} T H H\left(\ell_{p}\right) \cong E\left(\lambda_{1}, \lambda_{2}\right) \otimes P(\mu) .
$$

The induced map $V(1)_{*} T H H\left(\ell_{p}\right) \rightarrow V(1)_{*} T H H\left(k u_{p}\right)$ sends $\lambda_{1}$ and $\mu$ to the classes with same name, and $\lambda_{2}$ to the class $a_{1} b_{1}^{p-2}$.

Remark 6.2. In the sequel, we will frequently denote by $\lambda_{2}$ the class $a_{1} b_{1}^{p-2}$.

The $C_{p}$-Tate spectral sequence

$$
\hat{E}\left(C_{p}\right)_{s, t}^{2}=\hat{H}^{-s}\left(C_{p}, V(1)_{t} T H H\left(k u_{p}\right)\right) \Rightarrow V(1)_{s+t} T H H\left(k u_{p}\right)^{t C_{p}}
$$

has an $E_{2}$-term given by

$$
\hat{E}\left(C_{p}\right)^{2}=P\left(t, t^{-1}\right) \otimes E\left(u_{1}\right) \otimes V(1)_{*} T H H\left(k u_{p}\right)
$$

with $t$ in bidegree $(-2,0), u_{1}$ in bidegree $(-1,0)$, and $w \in V(1)_{t} T H H\left(k u_{p}\right)$ in bidegree $(0, t)$. Recall the description of $V(1)_{*} T H H\left(k u_{p}\right)$ given in (4.1).

Lemma 6.3. In the $C_{p}$ Tate spectral sequence $\hat{E}^{*}\left(C_{p}\right)$ the classes $\lambda_{1}, \lambda_{2}, b_{1}$ and $t \mu$ are infinite cycles. There are non-zero differentials

$$
\begin{aligned}
d^{2}\left(b_{i}\right) & =(1-i) a_{i} t \\
d^{2 p}\left(t^{1-p}\right) & \doteq \lambda_{1} \cdot t \\
d^{2 p^{2}}\left(t^{p-p^{2}}\right) & \doteq \lambda_{2} \cdot t^{p} \\
d^{2 p^{2}+1}\left(u_{1} \cdot t^{-p^{2}}\right) & \doteq t \mu
\end{aligned}
$$


with $0 \leqslant i \leqslant p-1$. The spectral sequence collapses at the $\hat{E}^{2 p^{2}+2}$-term, leaving

$$
\begin{aligned}
\hat{E}^{\infty}\left(C_{p}\right)= & P\left(t^{ \pm p^{2}}\right) \otimes E\left(\lambda_{1}, a_{1}\right) \otimes P_{p-1}\left(b_{1}\right) \\
& \oplus E\left(\lambda_{1}\right) \otimes P_{p-2}\left(b_{1}\right) \otimes \mathbb{F}_{p}\left\{a_{1} t^{j}, b_{1} t^{j} \mid v_{p}(j)=1\right\} .
\end{aligned}
$$

Remark 6.4. Beware that in the lemma above, the index $j$ appearing as a power of $t$ runs over all integers, positive or negative, with specified $p$-adic valuation. The same remark holds for the Lemmas 6.10 and 6.12 below, and also for the power $j$ of $\mu$ in Lemmas 6.11 and 6.13 below.

Proof. We know from [3, Proposition 4.8] that $t \mu$ is an infinite cycle. The classes $\lambda_{1}, \lambda_{2}$ and $b_{1}$ are also infinite cycles, see the argument given at the top of [3, page 21].

Let $d$ be Connes' operator (4.3) on $V(1)_{*} T H H\left(k u_{p}\right)$, and recall from above the notation $b_{0}=u$. We have

$$
d\left(b_{0}\right)=a_{0},
$$

and this relation is detected via the Hurewicz homomorphism in mod $(p)$ homology, see [1, §9]. It follows from [37, 3.3] that in the $S^{1}$ homotopy fixed-point spectral sequence

$$
E^{2}\left(S^{1}\right)=P(t) \otimes V(1)_{*} T H H\left(k u_{p}\right) \Rightarrow V(1)_{*} T H H\left(k u_{p}\right)^{h S^{1}}
$$

we have a $d^{2}$-differential

$$
d^{2}\left(b_{0}\right)=a_{0} t
$$

Since $E^{2}\left(S^{1}\right)$ injects into $\hat{E}^{2}\left(C_{p}\right)$ via $R^{h} F$, this differential is also present in $\hat{E}^{2}\left(C_{p}\right)$. The differentials $d^{2}\left(b_{i}\right)=(1-i) a_{i} t$ for $i \neq 0$ follow easily from the case $i=0$ and the multiplicative structure. Indeed $d^{2}(\mu)=0$ for degree reasons, and hence $d^{2}\left(u^{2} \mu\right)=2 u \mu a_{0} t$. From the relation $b_{i} b_{p-i}=u^{2} \mu$ we deduce that $d^{2}\left(b_{i}\right)=\alpha_{i} a_{i} t$ for some $\alpha_{i} \in \mathbb{F}_{p}$, because in $V(1)_{*} T H H\left(k u_{p}\right)$ the equation $x b_{p-i}=u \mu a_{0}$ has $x=a_{i}$ as unique (homogeneous) solution. First, notice that $0=d^{2}\left(b_{1}^{p-1}\right)=(p-1) \alpha_{1} \lambda_{2}$, so we have $\alpha_{1}=0$. Next, the relation $b_{1} b_{p-1}=u^{2} \mu$ implies that $\alpha_{p-1}=2$, while $b_{1} b_{i}=u b_{i+1}$ for $i \leqslant p-2$ implies that $\alpha_{i}=1+\alpha_{i+1}$. We deduce that $\alpha_{i}=1-i$, proving the claim on the $d^{2}$-differential, which leaves

$$
\hat{E}^{3}\left(C_{p}\right)=P\left(t^{ \pm 1}, t \mu\right) \otimes E\left(u_{1}, \lambda_{1}, a_{1}\right) \otimes P_{p-1}\left(b_{1}\right) .
$$

Lemma 6.1 determines the given next three non-zero differentials, by comparison with the case of the $\ell_{p}$ treated in [3, 5.5], and this takes care of the summand of $\delta$-weight zero. The only algebra generators of $\hat{E}^{3}\left(C_{p}\right)$ of non-zero $\delta$-weight are $a_{1}$ and $b_{1}$. We know that $b_{1}$ is an infinite cycle. In the $S^{1}$ Tate spectral sequence, using the known differentials, the tri-grading and the product, it is easy to see that $a_{1}$ survives to the $E^{2 p^{2}+2}$-term. Therefore $a_{1}$ also survives to the $E^{2 p^{2}+2}$-term in $\hat{E}^{*}\left(C_{p}\right)$, via the morphism of spectral sequences induced by $F$. The $d^{2 p}$ differential leaves

$$
\hat{E}^{2 p+1}\left(C_{p}\right)=P\left(t^{ \pm p}, t \mu\right) \otimes E\left(u_{1}, \lambda_{1}, a_{1}\right) \otimes P_{p-1}\left(b_{1}\right),
$$

and the $d^{2 p^{2}}$ differential leaves

$$
\begin{aligned}
\hat{E}^{2 p^{2}+1}\left(C_{p}\right)= & P\left(t^{ \pm p^{2}}, t \mu\right) \otimes E\left(u_{1}, \lambda_{1}, a_{1}\right) \otimes P_{p-1}\left(b_{1}\right) \\
& \oplus E\left(u_{1}, \lambda_{1}\right) \otimes P_{p-2}\left(b_{1}\right) \otimes P(t \mu) \otimes \mathbb{F}_{p}\left\{a_{1} t^{j}, b_{1} t^{j} \mid v_{p}(j)=1\right\},
\end{aligned}
$$

as can be computed using the relation $a_{1} \cdot b^{p-2}=\lambda_{2}$. Finally, $d^{2 p^{2}+1}$ leaves

$$
\begin{aligned}
\hat{E}^{2 p^{2}+2}\left(C_{p}\right)= & P\left(t^{ \pm p^{2}}\right) \otimes E\left(\lambda_{1}, a_{1}\right) \otimes P_{p-1}\left(b_{1}\right) \\
& \oplus E\left(\lambda_{1}\right) \otimes P_{p-2}\left(b_{1}\right) \otimes \mathbb{F}_{p}\left\{a_{1} t^{j}, b_{1} t^{j} \mid v_{p}(j)=1\right\},
\end{aligned}
$$


and at this stage the spectral sequence collapses for bidegree reasons.

Remark 6.5. The $d^{2}$-differential can also be determined by computing $d\left(b_{i}\right)$ for $i \geqslant 0$, using Connes' operator in Hochschild homology (c.f. [1, 3.4]).

Definition 6.6. We call a homomorphism of graded groups $k$-coconnected if it is an isomorphism in all dimensions greater than $k$ and injective in dimension $k$.

Proposition 6.7. The algebra map

$$
\left(\hat{\Gamma}_{1}\right)_{*}: V(1)_{*} T H H\left(k u_{p}\right) \rightarrow V(1)_{*} T H H\left(k u_{p}\right)^{t C_{p}}
$$

factorizes as the localization away from $\mu$, followed by an isomorphism

$$
V(1)_{*} T H H\left(k u_{p}\right)\left[\mu^{-1}\right] \rightarrow V(1)_{*} T H H\left(k u_{p}\right)^{t C_{p}}
$$

given by

$$
\lambda_{1} \mapsto \lambda_{1}, \mu \mapsto t^{-p^{2}}, b_{i} \mapsto t^{(1-i) p} b_{1}, \text { and } a_{i} \mapsto t^{(1-i) p} a_{1}
$$

for $0 \leqslant i \leqslant p-1$, up to some non-zero scalar multiples. In particular the map $\left(\hat{\Gamma}_{1}\right)_{*}$ is $(2 p-2)$-coconnected.

Proof. By naturality with respect to $\ell_{p} \rightarrow k u_{p}$ and by the computation of $\left(\hat{\Gamma}_{1}\right)_{*}$ for $\ell_{p}$ given in [3, Theorem 5.5], we know that the map $\left(\hat{\Gamma}_{1}\right)_{*}$ for $k u_{p}$ satisfies

$$
\lambda_{1} \mapsto \lambda_{1}, \quad \lambda_{2} \mapsto \lambda_{2} \text { and } \mu \mapsto t^{-p^{2}} .
$$

In $V(1)_{*} T H H\left(k u_{p}\right)$ we have multiplicative relations $u^{p-3} a_{i} b_{j}=\lambda_{2}$ for $i+j=p-1$, from which we deduce that $\left(\hat{\Gamma}_{1}\right)_{*}\left(u^{k} a_{i}\right) \neq 0$ and $\left(\hat{\Gamma}_{1}\right)_{*}\left(u^{k} b_{i}\right) \neq 0$ for any $0 \leqslant k \leqslant p-3$ and any $0 \leqslant i \leqslant p-1$. For degree reasons, this forces

$$
\left(\hat{\Gamma}_{1}\right)_{*}\left(a_{i}\right)=t^{(1-i) p} a_{1} \text { and }\left(\hat{\Gamma}_{1}\right)_{*}\left(b_{i}\right)=t^{(1-i) p} b_{1}
$$

up to some non-zero scalar multiples.

Corollary 6.8. The canonical maps

$$
\begin{aligned}
& \Gamma_{n}: T H H\left(k u_{p}\right)^{C_{p^{n}}} \rightarrow T H H\left(k u_{p}\right)^{h C_{p^{n}}}, \\
& \hat{\Gamma}_{n}: T H H\left(k u_{p}\right)^{C_{p^{n-1}}} \rightarrow T H H\left(k u_{p}\right)^{t C_{p^{n}}}, \\
& \Gamma: T F\left(k u_{p}\right) \rightarrow T H H\left(k u_{p}\right)^{h S^{1}}, \\
& \hat{\Gamma}: T F\left(k u_{p}\right) \rightarrow T H H\left(k u_{p}\right)^{t S^{1},}
\end{aligned}
$$

for $n \geqslant 1$ all induce $(2 p-2)$-coconnected maps in $V(1)$-homotopy.

Proof. The claims for $\Gamma_{n}$ and $\hat{\Gamma}_{n}$ follow from Proposition 6.7 and the generalization of a theorem of Tsalidis [43] given in [9]. The claims for $\Gamma$ and $\hat{\Gamma}$ follow by passage to homotopy limits.

Definition 6.9. Let $r(n)=0$ for all $n \leqslant 0$, and let $r(n)=p^{n}+r(n-2)$ for all $n \geqslant 1$. Thus $r(2 n-1)=p^{2 n-1}+\cdots+p$ (odd powers) and $r(2 n)=p^{2 n}+\cdots+p^{2}$ (even powers).

Lemma 6.10. In the $C_{p^{n}}$ Tate spectral sequence $\hat{E}^{*}\left(C_{p^{n}}\right)$ the classes $\lambda_{1}, \lambda_{2}, b_{1}$ and $t \mu$ are infinite cycles. There are non-zero differentials

$$
\begin{aligned}
d^{2}\left(b_{i}\right) & =(1-i) a_{i} t \\
d^{2 p}\left(t^{1-p}\right) & \doteq \lambda_{1} \cdot t \\
d^{2 p^{2}}\left(t^{p-p^{2}}\right) & \doteq \lambda_{2} \cdot t^{p}
\end{aligned}
$$


with $0 \leqslant i \leqslant p-1$, leaving

$$
\begin{aligned}
\hat{E}^{2 p^{2}+1}\left(C_{p^{n}}\right)= & P\left(t^{ \pm p^{2}}\right) \otimes E\left(u_{n}, \lambda_{1}, a_{1}\right) \otimes P_{p-1}\left(b_{1}\right) \otimes P(t \mu) \\
& \oplus E\left(u_{n}, \lambda_{1}\right) \otimes P_{p-2}\left(b_{1}\right) \otimes P(t \mu) \otimes \mathbb{F}_{p}\left\{a_{1} t^{j}, b_{1} t^{j} \mid v_{p}(j)=1\right\} .
\end{aligned}
$$

If $n \geqslant 2$, then for each $1 \leqslant k \leqslant n-1$ there is a triple of non-zero differentials

$$
\begin{aligned}
d^{2 r(2 k)+2}\left(b_{1} t^{j}\right) & \doteq a_{1} t^{j} \cdot t^{p^{2 k}} \cdot(t \mu)^{r(2 k-2)+1} \\
d^{2 r(2 k+1)}\left(t^{p^{2 k}-p^{2 k+1}}\right) & \doteq \lambda_{1} \cdot t^{p^{2 k}} \cdot(t \mu)^{r(2 k-1)} \\
d^{2 r(2 k+2)}\left(t^{p^{2 k+1}-p^{2 k+2}}\right) & \doteq \lambda_{2} \cdot t^{p^{2 k+1}} \cdot(t \mu)^{r(2 k)}
\end{aligned}
$$

with $v_{p}(j)=2 k-1$, leaving

$$
\begin{aligned}
\hat{E}^{2 r(2 k+2)+1}\left(C_{p^{n}}\right)= & P\left(t^{ \pm p^{2 k+2}}\right) \otimes E\left(u_{n}, \lambda_{1}, a_{1}\right) \otimes P_{p-1}\left(b_{1}\right) \otimes P(t \mu) \\
& \oplus E\left(u_{n}, \lambda_{1}\right) \otimes P_{p-2}\left(b_{1}\right) \otimes P(t \mu) \otimes \mathbb{F}_{p}\left\{a_{1} t^{j}, b_{1} t^{j} \mid v_{p}(j)=2 k+1\right\} \\
& \oplus \bigoplus_{1 \leqslant m \leqslant k} \hat{T}_{m}\left(C_{p^{n}}\right),
\end{aligned}
$$

where

$$
\begin{aligned}
\hat{T}_{m}\left(C_{p^{n}}\right)= & E\left(u_{n}, \lambda_{1}\right) \otimes P_{r(2 m)}(t \mu) \otimes \mathbb{F}_{p}\left\{\lambda_{2} t^{j} \mid v_{p}(j)=2 m+1\right\} \\
& \oplus E\left(u_{n}, a_{1}\right) \otimes P_{p-1}\left(b_{1}\right) \otimes P_{r(2 m-1)}(t \mu) \otimes \mathbb{F}_{p}\left\{\lambda_{1} t^{j} \mid v_{p}(j)=2 m\right\} \\
& \oplus E\left(u_{n}, \lambda_{1}\right) \otimes P_{p-2}\left(b_{1}\right) \otimes P_{r(2 m-2)+1}(t \mu) \otimes \mathbb{F}_{p}\left\{a_{1} t^{j} \mid v_{p}(j)=2 m-1\right\} .
\end{aligned}
$$

For $n \geqslant 1$, there is a last non-zero differential

$$
d^{2 r(2 n)+1}\left(u_{n} \cdot t^{-p^{2 n}}\right) \doteq(t \mu)^{r(2 n-2)+1}
$$

after which the spectral sequence collapses, leaving

$$
\begin{aligned}
\hat{E}^{\infty}\left(C_{p^{n}}\right)= & P\left(t^{ \pm p^{2 n}}\right) \otimes E\left(\lambda_{1}, a_{1}\right) \otimes P_{p-1}\left(b_{1}\right) \otimes P_{r(2 n-2)+1}(t \mu) \\
& \oplus E\left(\lambda_{1}\right) \otimes P_{p-2}\left(b_{1}\right) \otimes P_{r(2 n-2)+1}(t \mu) \otimes \mathbb{F}_{p}\left\{a_{1} t^{j}, b_{1} t^{j} \mid v_{p}(j)=2 n-1\right\} \\
& \oplus \bigoplus_{1 \leqslant m \leqslant n-1} \hat{T}_{m}\left(C_{p^{n}}\right) .
\end{aligned}
$$

Next, we describe the $C_{p^{n}}$ homotopy fixed-point spectral sequence $E^{*}\left(C_{p^{n}}\right)$. It is algebraically easier to describe the $E^{r}$-terms of the $C_{p^{n}}$ homotopy fixed-point spectral sequence for $T H H\left(k u_{p}\right)^{t C_{p}}$, which we denote abusively by

$$
\mu^{-1} E^{*}\left(C_{p^{n}}\right) \Rightarrow V(1)_{*}\left(T H H\left(k u_{p}\right)^{t C_{p}}\right)^{h C_{p^{n}}},
$$

compare with [3, page 23]. We know from Proposition 6.7 that the map

$$
\hat{\Gamma}_{1}^{h C_{p^{n}}}: T H H\left(k u_{p}\right)^{h C_{p^{n}}} \rightarrow\left(T H H\left(k u_{p}\right)^{t C_{p}}\right)^{h C_{p^{n}}}
$$

induces a morphism of spectral sequences

$$
E^{*}\left(C_{p^{n}}\right) \rightarrow \mu^{-1} E^{*}\left(C_{p^{n}}\right)
$$

which on $E^{2}$-terms (but not on higher terms) indeed corresponds to inverting $\mu$. By the same Proposition and by strong convergence of the spectral sequences, the map $\hat{\Gamma}_{1}^{h C_{p^{n}}}$ induces a $(2 p-2)$-coconnected homomorphism in $V(1)$-homotopy. 
Lemma 6.11. In the $C_{p^{n}}$ homotopy fixed-point spectral sequence $\mu^{-1} E^{*}\left(C_{p^{n}}\right)$ the classes $\lambda_{1}, \lambda_{2}, b_{1}$ and $t \mu$ are infinite cycles. There are non-zero differentials

$$
\begin{aligned}
d^{2}\left(b_{i}\right) & =(1-i) a_{i} t \\
d^{2 p}\left(\mu^{p-1}\right) & \doteq \lambda_{1} \cdot \mu^{-1} \cdot(t \mu)^{p} \\
d^{2 p^{2}}\left(\mu^{p^{2}-p}\right) & \doteq \lambda_{2} \cdot \mu^{-p} \cdot(t \mu)^{p^{2}}
\end{aligned}
$$

with $0 \leqslant i \leqslant p-1$, leaving

$$
\begin{aligned}
\mu^{-1} E^{2 p^{2}+1}\left(C_{p^{n}}\right)= & P\left(\mu^{ \pm p^{2}}\right) \otimes E\left(u_{n}, \lambda_{1}, a_{1}\right) \otimes P_{p-1}\left(b_{1}\right) \otimes P(t \mu) \\
& \oplus E\left(u_{n}, \lambda_{1}\right) \otimes P_{p-2}\left(b_{1}\right) \otimes P(t \mu) \otimes \mathbb{F}_{p}\left\{a_{1} \mu^{j}, b_{1} \mu^{j} \mid v_{p}(j)=1\right\} \\
& \oplus T_{1}\left(C_{p^{n}}\right)
\end{aligned}
$$

where

$$
\begin{aligned}
T_{1}\left(C_{p^{n}}\right)= & E\left(u_{n}, \lambda_{1}\right) \otimes P_{p^{2}}(t \mu) \otimes \mathbb{F}_{p}\left\{\lambda_{2} \mu^{j} \mid v_{p}(j)=1\right\} \\
& \oplus E\left(u_{n}, a_{1}\right) \otimes P_{p-1}\left(b_{1}\right) \otimes P_{p}(t \mu) \otimes \mathbb{F}_{p}\left\{\lambda_{1} \mu^{j} \mid v_{p}(j)=0\right\} \\
& \oplus E\left(u_{n}, \lambda_{1}\right) \otimes P_{p-2}\left(b_{1}\right) \otimes P\left(\mu^{ \pm 1}\right) \otimes \mathbb{F}_{p}\left\{a_{i} \mid 0 \leqslant i \leqslant p-1, i \neq 1\right\} .
\end{aligned}
$$

If $n \geqslant 2$, then for each $2 \leqslant k \leqslant n$ there is a triple of non-zero differentials

$$
\begin{aligned}
d^{2 r(2 k-2)+2}\left(b_{1} \mu^{j}\right) & \doteq a_{1} \mu^{j} \cdot \mu^{-p^{2 k-2}} \cdot(t \mu)^{r(2 k-2)+1} \\
d^{2 r(2 k-1)}\left(\mu^{p^{2 k-1}-p^{2 k-2}}\right) & \doteq \lambda_{1} \cdot \mu^{-p^{2 k-2}} \cdot(t \mu)^{r(2 k-1)} \\
d^{2 r(2 k)}\left(\mu^{p^{2 k}-p^{2 k-1}}\right) & \doteq \lambda_{2} \cdot \mu^{-p^{2 k-1}} \cdot(t \mu)^{r(2 k)}
\end{aligned}
$$

with $v_{p}(j)=2 k-3$, leaving

$$
\begin{aligned}
\mu^{-1} E^{2 r(2 k)+1}\left(C_{p^{n}}\right)= & P\left(\mu^{ \pm p^{2 k}}\right) \otimes E\left(u_{n}, \lambda_{1}, a_{1}\right) \otimes P_{p-1}\left(b_{1}\right) \otimes P(t \mu) \\
& \oplus E\left(u_{n}, \lambda_{1}\right) \otimes P_{p-2}\left(b_{1}\right) \otimes P(t \mu) \otimes \mathbb{F}_{p}\left\{a_{1} \mu^{j}, b_{1} \mu^{j} \mid v_{p}(j)=2 k-1\right\} \\
& \oplus \bigoplus_{1 \leqslant m \leqslant k} T_{m}\left(C_{p^{n}}\right),
\end{aligned}
$$

where for $m \geqslant 2$ we have

$$
\begin{aligned}
T_{m}\left(C_{p^{n}}\right)= & E\left(u_{n}, \lambda_{1}\right) \otimes P_{r(2 m)}(t \mu) \otimes \mathbb{F}_{p}\left\{\lambda_{2} \mu^{j} \mid v_{p}(j)=2 m-1\right\} \\
& \oplus E\left(u_{n}, a_{1}\right) \otimes P_{p-1}\left(b_{1}\right) \otimes P_{r(2 m-1)}(t \mu) \otimes \mathbb{F}_{p}\left\{\lambda_{1} \mu^{j} \mid v_{p}(j)=2 m-2\right\} \\
& \oplus E\left(u_{n}, \lambda_{1}\right) \otimes P_{p-2}\left(b_{1}\right) \otimes P_{r(2 m-2)+1}(t \mu) \otimes \mathbb{F}_{p}\left\{a_{1} \mu^{j} \mid v_{p}(j)=2 m-3\right\} .
\end{aligned}
$$

For $n \geqslant 1$, there is a last non-zero differential

$$
d^{2 r(2 n)+1}\left(u_{n} \cdot \mu^{p^{2 n}}\right) \doteq(t \mu)^{r(2 n)+1}
$$

after which the spectral sequence collapses, leaving

$$
\begin{aligned}
\mu^{-1} E^{\infty}\left(C_{p^{n}}\right)= & P\left(\mu^{ \pm p^{2 n}}\right) \otimes E\left(\lambda_{1}, a_{1}\right) \otimes P_{p-1}\left(b_{1}\right) \otimes P_{r(2 n)+1}(t \mu) \\
& \oplus E\left(\lambda_{1}\right) \otimes P_{p-2}\left(b_{1}\right) \otimes P_{r(2 n)+1}(t \mu) \otimes \mathbb{F}_{p}\left\{a_{1} \mu^{j}, b_{1} \mu^{j} \mid v_{p}(j)=2 n-1\right\} \\
& \oplus \bigoplus_{1 \leqslant m \leqslant n} T_{m}\left(C_{p^{n}}\right) .
\end{aligned}
$$


Proof. We prove these two lemmas by induction on $n$, showing that Lemma 6.10 for $C_{p^{n}}$ implies Lemma 6.11 for $C_{p^{n}}$, which in turn implies Lemma 6.10 for $C_{p^{n+1}}$. The induction starts with Lemma 6.10 for $C_{p}$, which is the content of Lemma 6.3. Let us therefore assume given $n \geqslant 1$ such that Lemma 6.10 holds for $C_{p^{n}}$. The homotopy restriction map

$$
R^{h}: T H H\left(k u_{p}\right)^{h C_{p^{n}}} \rightarrow T H H\left(k u_{p}\right)^{t C_{p^{n}}}
$$

induces a morphism of spectral sequences $\left(R^{h}\right)^{*}: E^{*}\left(C_{p^{n}}\right) \rightarrow \hat{E}^{*}\left(C_{p^{n}}\right)$, which at the $E^{2}$-terms corresponds to inverting the class $t \in E_{-2,0}^{2}\left(C_{p^{n}}\right)$,

$$
\left(R^{h}\right)^{2}: E^{2}\left(C_{p^{n}}\right) \subset E^{2}\left(C_{p^{n}}\right)\left[t^{-1}\right] \cong \hat{E}^{2}\left(C_{p^{n}}\right),
$$

and can be pictured as the inclusion of the second quadrant into the upper-half plane. As we will see below, although $\left(R^{h}\right)^{r}$ is not injective for $r \geqslant 3$, it detects all the non-trivial differentials of $E^{r}\left(C_{p^{n}}\right)$. Taking into account the multiplicative structure and the fact that $\lambda_{1}, \lambda_{2}, b_{1}$ and $t \mu$ are infinite cycles, we claim that these differential are given by

$$
\begin{aligned}
d^{2}\left(b_{i}\right) & =(1-i) a_{i} t \\
d^{2 p}(t) & \doteq \lambda_{1} \cdot t^{1+p} \\
d^{2 p^{2}}\left(t^{p}\right) & \doteq \lambda_{2} \cdot t^{p+p^{2}}
\end{aligned}
$$

with $0 \leqslant i \leqslant p-1$,

$$
\begin{aligned}
d^{2 r(2 k)+2}\left(b_{1} t^{j}\right) & \doteq a_{1} t^{j} \cdot t^{p^{2 k}} \cdot(t \mu)^{r(2 k-2)+1} \\
d^{2 r(2 k+1)}\left(t^{p^{2 k}}\right) & \doteq \lambda_{1} \cdot t^{p^{2 k}+p^{2 k+1}} \cdot(t \mu)^{r(2 k-1)} \\
d^{2 r(2 k+2)}\left(t^{p^{2 k+1}}\right) & \doteq \lambda_{2} \cdot t^{p^{2 k+1}+p^{2 k+2}} \cdot(t \mu)^{r(2 k)}
\end{aligned}
$$

if $n \geqslant 2,1 \leqslant k \leqslant n-1$ and $v_{p}(j)=2 k-1$ with $i \geqslant 0$, and finally

$$
d^{2 r(2 n)+1}\left(u_{n}\right) \doteq(t \mu)^{r(2 n-2)+1} \cdot t^{p^{2 n}} .
$$

To prove this claim, we assume that some $r \geqslant 2$ is given, and that $E^{r}\left(C_{p^{n}}\right)$ has been computed using the differentials $d^{r^{\prime}}$ above with $r^{\prime}<r$. The class $t \mu$ is an infinite cycle, and $E^{r}\left(C_{p^{n}}\right)$ is a $P(t \mu)$-module. Our choice of generators induces a decomposition $E^{r}\left(C_{p^{n}}\right) \cong$ $F^{r}\left(C_{p^{n}}\right) \oplus T^{r}\left(C_{p^{n}}\right)$, where $F^{r}\left(C_{p^{n}}\right)$ is a free $P(t \mu)$-module and $T^{r}\left(C_{p^{n}}\right)$ is a $t \mu$-torsion module. By inspection, the non-zero elements of $T^{r}\left(C_{p^{n}}\right)$ are concentrated in filtration degrees $s$ with $-r<s \leqslant 0$, so they cannot be boundaries. They cannot support non-zero differentials either since a $t \mu$-torsion class cannot map to a non-torsion class. Thus the differential $d^{r}$ maps $F^{r}\left(C_{p^{n}}\right)$ to itself and $T^{r}\left(C_{p^{n}}\right)$ to zero. The morphism $\left(R^{h}\right)^{r}$ maps $F^{r}\left(C_{p^{n}}\right)$ injectively into $\hat{E}^{r}\left(C_{p^{n}}\right)$, and it therefore detects the non-zero differentials of $E^{r}\left(C_{p^{n}}\right)$ as the non-zero differential of $\hat{E}^{r}\left(C_{p^{n}}\right)$ which lie in the second quadrant. These are precisely the differentials given above. By induction on $r$, this determines all the nontrivial differentials of $E^{*}\left(C_{p^{n}}\right)$. In the $\mu$-inverted homotopy fixed-point spectral sequence $\mu^{-1} E^{*}\left(C_{p^{n}}\right)$, these can be rewritten as the claimed differentials. This proves Lemma 6.11 for $C_{p^{n}}$.

We now turn to the proof of Lemma 6.10 for $C_{p^{n+1}}$. In the Tate spectral sequence $\hat{E}^{*}\left(C_{p^{n}}\right)$ the first non-zero differential of odd length originating from a column of odd $s$-filtration is $d^{2 r(2 n)+1}$. By [3], Lemma 5.2] the spectral sequences $\hat{E}^{*}\left(C_{p^{n}}\right)$ and $\hat{E}^{*}\left(C_{p^{n+1}}\right)$ are abstractly isomorphic up to the $E^{2 r(2 n)+1}$-term included. The Frobenius map

$$
F: T H H\left(k u_{p}\right)^{t C_{p^{n+1}}} \rightarrow T H H\left(k u_{p}\right)^{t C_{p^{n}}}
$$


induces a morphism of the corresponding Tate spectral sequences, which on $E^{r}$-terms with $2 \leqslant r \leqslant 2 r(2 n)+1$ maps the columns of even $s$-filtration isomorphically. This detects all the claimed differentials of $\hat{E}^{r}\left(C_{p^{n+1}}\right)$ for $2 \leqslant r \leqslant 2 r(2 n)$, and leaves

$$
\hat{E}^{2 r(2 n)+1}\left(C_{p^{n+1}}\right)=\hat{F}^{2 r(2 n)+1}\left(C_{p^{n+1}}\right) \oplus \bigoplus_{m=1}^{n-1} \hat{T}_{m}\left(C_{p^{n+1}}\right),
$$

where $\hat{F}^{2 r(2 n)+1}\left(C_{p^{n+1}}\right)$ is the $t \mu$-torsion free summand

$$
\begin{aligned}
& \hat{F}^{2 r(2 n)+1}\left(C_{p^{n+1}}\right)=P\left(t^{ \pm p^{2 n}}\right) \otimes E\left(u_{n+1}, \lambda_{1}\right) \otimes P(t \mu) \\
& \quad \otimes\left(P_{p-1}\left(b_{1}\right) \otimes E\left(a_{1}\right) \oplus P_{p-2}\left(b_{1}\right) \otimes \mathbb{F}_{p}\left\{a_{1} t^{-i p^{2 n-1}}, b_{1} t^{-i p^{2 n-1}} \mid 0<i<p\right\}\right) .
\end{aligned}
$$

The non-zero $t \mu$-torsion elements of $\hat{E}^{2 r(2 n)+1}\left(C_{p^{n+1}}\right)$ are concentrated in internal degrees $t$ with $0 \leqslant t<2 r(2 n)$. In particular these elements cannot be boundaries, and they cannot map to non- $t \mu$-torsion elements. As in the case of the homotopy fixed-point spectral sequence above, we deduce that for $r \geqslant 2 r(2 n)+1$ the differential $d^{r}$ can only affect the summand $\hat{F}^{2 r(2 n)+1}\left(C_{p^{n+1}}\right)$. By Lemma 6.1 the summand of $\delta$-weight 0 of $\hat{E}^{*}\left(C_{p^{n+1}}\right)$ is equal to the image of the injective morphism of spectral sequences

$$
\hat{E}^{*}\left(C_{p^{n+1}}, \ell_{p}\right) \rightarrow \hat{E}^{*}\left(C_{p^{n+1}}, k u_{p}\right)=\hat{E}^{*}\left(C_{p^{n+1}}\right)
$$

induced by the map $\ell_{p} \rightarrow k u_{p}$. Therefore, by [3, Theorem 6.1], the differentials affecting the summand of $\delta$-weight 0 of $\hat{F}^{2 r(2 n)+1}\left(C_{p^{n+1}}\right)$ at a later stage are given by

$$
\begin{aligned}
d^{2 r(2 n+1)}\left(t^{p^{2 n}-p^{2 n+1}}\right) & \doteq \lambda_{1} \cdot t^{p^{2 n}} \cdot(t \mu)^{r(2 n-1)} \\
d^{2 r(2 n+2)}\left(t^{p^{2 n+1}-p^{2 n+2}}\right) & \doteq \lambda_{2} \cdot t^{p^{2 n+1}} \cdot(t \mu)^{r(2 n)} \\
d^{2 r(2 n+2)+1}\left(u_{n+1} \cdot t^{-p^{2 n+2}}\right) & \doteq(t \mu)^{r(2 n)+1},
\end{aligned}
$$

together with the multiplicative structure and the fact that $t \mu$ is an infinite cycle. It remains to prove that from the $E^{2 r(2 n)+1}$-term on, the only non-zero differentials supported by homogeneous algebra generators of $\delta$-weight 1 are given by

$$
d^{2 r(2 n)+2}\left(b_{1} t^{j}\right) \doteq a_{1} t^{j} \cdot t^{p^{2 n}} \cdot(t \mu)^{r(2 n-2)+1}
$$

for $v_{p}(j)=2 n-1$. First, notice that for tri-degree reasons $d^{2 r(2 n)+1}=0$, so that $\hat{F}^{2 r(2 n)+2}\left(C_{p^{n+1}}\right)=\hat{F}^{2 r(2 n)+1}\left(C_{p^{n+1}}\right)$. To detect the differential (6.4) we make use of the $(2 p-2)$-coconnected map

$$
\left(\hat{\Gamma}_{n+1}\right)_{*}: V(1)_{*} T H H\left(k u_{p}\right)^{C_{p^{n}}} \rightarrow V(1)_{*} T H H\left(k u_{p}\right)^{t C_{p^{n+1}}},
$$

and argue as in [3, proof of 6.1]. There is a commutative diagram

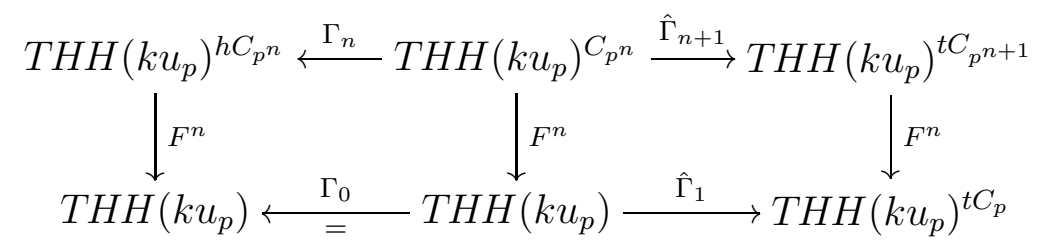

where the vertical arrows are the $n$-fold Frobenius maps. The left-hand Frobenius is given in $V(1)$-homotopy on the associated graded by the edge homomorphism

$$
E_{*, *}^{\infty}\left(C_{p^{n}}\right) \rightarrow E_{0, *}^{\infty}\left(C_{p^{n}}\right) \subset E_{0, *}^{2}\left(C_{p^{n}}\right)=V(1)_{*} T H H\left(k u_{p}\right)
$$


which is known by induction hypothesis. For each $0<\ell<p$ there is a direct summand

$$
P_{r(2 n-2)+1}(t \mu)\left\{a_{1} \mu^{\ell p^{2 n-3}}\right\} \subset E_{*, *}^{\infty}\left(C_{p^{n}}\right),
$$

and $a_{1} \mu^{\ell p^{2 n-3}}$ maps by $F_{*}^{n}$ to the class with same name in $V(1)_{*} T H H\left(k u_{p}\right)$. Since $\left(\Gamma_{n}\right)_{*}$ is $(2 p-2)$-coconnected, there is a class $x_{\ell} \in V(1)_{*} T H H\left(k u_{p}\right)^{C_{p^{n}}}$ with $F_{*}^{n}\left(x_{\ell}\right)=a_{1} \mu^{\ell p^{2 n-3}}$ in $V(1)_{*} T H H\left(k u_{p}\right)$. In $E^{\infty}\left(C_{p^{n}}\right)$ we have no non-zero class of same total degree, same $\delta$-weight and lower $s$-filtration than

$$
(t \mu)^{r(2 n-2)+1} \cdot a_{1} \mu^{\ell p^{2 n-3}}
$$

which forces $v_{2}^{r(2 n-2)+1} x_{\ell}=0$ in $V(1)_{*} T H H\left(k u_{p}\right)^{C_{p^{n}}}$. By Proposition 6.7, the class $\left(\hat{\Gamma}_{1} F^{n}\right)_{*}\left(x_{\ell}\right)$ is represented by $a_{1} t^{-\ell p^{2 n-1}} \in \hat{E}^{\infty}\left(C_{p}\right)$, and therefore $\left(\hat{\Gamma}_{n+1}\right)_{*}\left(x_{\ell}\right)$ must be detected in $s$-filtration $2 \ell p^{2 n-1}$ or higher. The only suitable class in $\hat{E}^{2 r(2 n)+2}\left(C_{p^{n+1}}\right)$ is $a_{1} t^{-\ell p^{2 n-1}}$, which therefore is a permanent cycle representing $\left(\hat{\Gamma}_{n+1}\right)_{*}\left(x_{\ell}\right)$. Notice for later use that the same argument shows that

$$
a_{1} \in \hat{E}_{0,2 p+3}^{2 r(2 n)+2}\left(C_{p^{n+1}}\right)
$$

is a permanent cycle. The map $\left(\hat{\Gamma}_{n+1}\right)_{*}$ is an isomorphism in degrees larger than $2 p-2$, and the relation $v_{2}^{r(2 n-2)+1}\left(\hat{\Gamma}_{n+1}\right)_{*}\left(x_{\ell}\right)=0$ implies that the infinite cycle $(t \mu)^{r(2 n-2)+1}$. $a_{1} t^{-\ell p^{2 n-1}}$, of total degree $2 p^{2 n}+2 \ell p^{2 n-1}+2 p+1$ and of $\delta$-weight 1 , is a boundary. On the other hand, the component of $\hat{E}^{2 r(2 n)+1}\left(C_{p^{n+1}}\right)$ of total degree $2 p^{2 n}+2 \ell p^{2 n-1}+2 p+2$, of $\delta$-weight 1 and of $s$-filtration degree exceeding by at least $2 r(2 n)+2$ the $s$-filtration degree of $(t \mu)^{r(2 n-2)+1} \cdot a_{1} t^{-\ell p^{2 n-1}}$ reduces to

$$
\mathbb{F}_{p}\left\{b_{1} t^{-\ell p^{2 n-1}} \cdot t^{-p^{2 n}}\right\}
$$

This proves the existence of a non-zero differential

$$
d^{2 r(2 n)+2}\left(b_{1} t^{-\ell p^{2 n-1}} \cdot t^{-p^{2 n}}\right) \doteq(t \mu)^{r(2 n-2)+1} \cdot a_{1} t^{-\ell p^{2 n-1}}
$$

for $0<\ell<p$. Since $t^{p^{2 n}}$ is a unit and a cycle we obtain the claimed differentials (6.4). This leaves

$$
\begin{aligned}
\hat{E}^{2 r(2 n)+3}\left(C_{p^{n+1}}\right) & =\hat{F}^{2 r(2 n)+3}\left(C_{p^{n+1}}\right) \\
& \oplus E\left(u_{n+1}, \lambda_{1}\right) \otimes P_{p-2}\left(b_{1}\right) \otimes P_{r(2 n-2)+1}(t \mu) \otimes \mathbb{F}_{p}\left\{a_{1} t^{j} \mid v_{p}(j)=2 n-1\right\} \\
& \oplus \bigoplus_{m=1}^{n-1} \hat{T}_{m}\left(C_{p^{n+1}}\right),
\end{aligned}
$$

with a $t \mu$-torsion free summand

$$
F^{2 r(2 n)+3}\left(C_{p^{n+1}}\right)=P\left(t^{ \pm p^{2 n}}\right) \otimes E\left(u_{n+1}, \lambda_{1}, a_{1}\right) \otimes P_{p-1}\left(b_{1}\right) \otimes P(t \mu) .
$$

Again, further differentials can only affect the summand $F^{2 r(2 n)+3}\left(C_{p^{n+1}}\right)$. Since $b_{1}$ and $a_{1}$ are infinite cycles, the next non-zero differentials are $d^{2 r(2 n+1)}$ and $d^{2 r(2 n+2)}$, as given in (6.3), leaving

$$
\hat{E}^{2 r(2 n+2)+1}\left(C_{p^{n+1}}\right)=\hat{F}^{2 r(2 n+2)+1}\left(C_{p^{n+1}}\right) \oplus \bigoplus_{m=1}^{n} \hat{T}_{m}\left(C_{p^{n+1}}\right),
$$


with

$$
\begin{aligned}
& \hat{F}^{2 r(2 n+2)+1}\left(C_{p^{n+1}}\right)=P\left(t^{ \pm p^{2 n+2}}\right) \otimes E\left(u_{n+1}, \lambda_{1}\right) \otimes P(t \mu) \\
& \quad \otimes\left(P_{p-1}\left(b_{1}\right) \otimes E\left(a_{1}\right) \oplus P_{p-2}\left(b_{1}\right) \otimes \mathbb{F}_{p}\left\{a_{1} t^{-i p^{2 n+1}}, b_{1} t^{-i p^{2 n+1}} \mid 0<i<p\right\}\right) .
\end{aligned}
$$

Notice that for tri-degree reasons, the classes $a_{1} t^{-i p^{2 n+1}}$ and $b_{1} t^{-i p^{2 n+1}}$ are cycles at the $E^{2 r(2 n+2)+1}$-stage. The third differential of (6.3) remains, after which the spectral sequence collapses for bidegree reasons, leaving

$$
\begin{aligned}
\hat{E}^{\infty}\left(C_{p^{n+1}}\right)= & P\left(t^{ \pm p^{2(n+1)}}\right) \otimes E\left(\lambda_{1}, a_{1}\right) \otimes P_{p-1}\left(b_{1}\right) \otimes P_{r(2 n)+1}(t \mu) \\
& \oplus E\left(\lambda_{1}\right) \otimes P_{p-2}\left(b_{1}\right) \otimes P_{r(2 n)+1}(t \mu) \otimes \mathbb{F}_{p}\left\{a_{1} t^{j}, b_{1} t^{j} \mid v_{p}(j)=2 n+1\right\} \\
& \oplus \bigoplus_{1 \leqslant m \leqslant n} \hat{T}_{m}\left(C_{p^{n+1}}\right),
\end{aligned}
$$

as claimed. This completes the induction step and the proof of Lemmas 6.10 and 6.11 .

Taking the limit over the Frobenius maps we obtain the following two lemmas.

Lemma 6.12. The associated graded $\hat{E}^{\infty}\left(S^{1}\right)$ of $V(1)_{*} T H H\left(k u_{p}\right)^{t S^{1}}$ is given by

$$
\hat{E}^{\infty}\left(S^{1}\right)=E\left(\lambda_{1}, a_{1}\right) \otimes P_{p-1}\left(b_{1}\right) \otimes P(t \mu) \oplus \bigoplus_{m \geqslant 1} \hat{T}_{m}\left(S^{1}\right)
$$

where

$$
\begin{aligned}
\hat{T}_{m}\left(S^{1}\right)= & E\left(\lambda_{1}\right) \otimes P_{r(2 m)}(t \mu) \otimes \mathbb{F}_{p}\left\{\lambda_{2} t^{j} \mid v_{p}(j)=2 m+1\right\} \\
& \oplus E\left(a_{1}\right) \otimes P_{p-1}\left(b_{1}\right) \otimes P_{r(2 m-1)}(t \mu) \otimes \mathbb{F}_{p}\left\{\lambda_{1} t^{j} \mid v_{p}(j)=2 m\right\} \\
& \oplus E\left(\lambda_{1}\right) \otimes P_{p-2}\left(b_{1}\right) \otimes P_{r(2 m-2)+1}(t \mu) \otimes \mathbb{F}_{p}\left\{a_{1} t^{j} \mid v_{p}(j)=2 m-1\right\} .
\end{aligned}
$$

Lemma 6.13. The associated graded $E^{\infty}\left(S^{1}\right)$ of $V(1)_{*} T H H\left(k u_{p}\right)^{h S^{1}}$ is mapped by a $(2 p-2)$-coconnected homomorphism to

$$
\mu^{-1} E^{\infty}\left(S^{1}\right)=E\left(\lambda_{1}, a_{1}\right) \otimes P_{p-1}\left(b_{1}\right) \otimes P(t \mu) \oplus \bigoplus_{m \geqslant 1} T_{m}\left(S^{1}\right)
$$

where

$$
\begin{aligned}
T_{1}\left(S^{1}\right)= & E\left(\lambda_{1}\right) \otimes P_{p^{2}}(t \mu) \otimes \mathbb{F}_{p}\left\{\lambda_{2} \mu^{j} \mid v_{p}(j)=1\right\} \\
& \oplus E\left(a_{1}\right) \otimes P_{p-1}\left(b_{1}\right) \otimes P_{p}(t \mu) \otimes \mathbb{F}_{p}\left\{\lambda_{1} \mu^{j} \mid v_{p}(j)=0\right\} \\
& \oplus E\left(\lambda_{1}\right) \otimes P_{p-2}\left(b_{1}\right) \otimes P\left(\mu^{ \pm 1}\right) \otimes \mathbb{F}_{p}\left\{a_{i} \mid 0 \leqslant i \leqslant p-1, i \neq 1\right\}
\end{aligned}
$$

and, for $m \geqslant 2$,

$$
\begin{aligned}
T_{m}\left(S^{1}\right)= & E\left(\lambda_{1}\right) \otimes P_{r(2 m)}(t \mu) \otimes \mathbb{F}_{p}\left\{\lambda_{2} \mu^{j} \mid v_{p}(j)=2 m-1\right\} \\
& \oplus E\left(a_{1}\right) \otimes P_{p-1}\left(b_{1}\right) \otimes P_{r(2 m-1)}(t \mu) \otimes \mathbb{F}_{p}\left\{\lambda_{1} \mu^{j} \mid v_{p}(j)=2 m-2\right\} \\
& \oplus E\left(\lambda_{1}\right) \otimes P_{p-2}\left(b_{1}\right) \otimes P_{r(2 m-2)+1}(t \mu) \otimes \mathbb{F}_{p}\left\{a_{1} \mu^{j} \mid v_{p}(j)=2 m-3\right\} .
\end{aligned}
$$




\section{Topological CyClic homology}

We now evaluate the restriction map $R: T F\left(k u_{p}\right) \rightarrow T F\left(k u_{p}\right)$ in $V(1)$-homotopy. Consider the homotopy commutative diagram

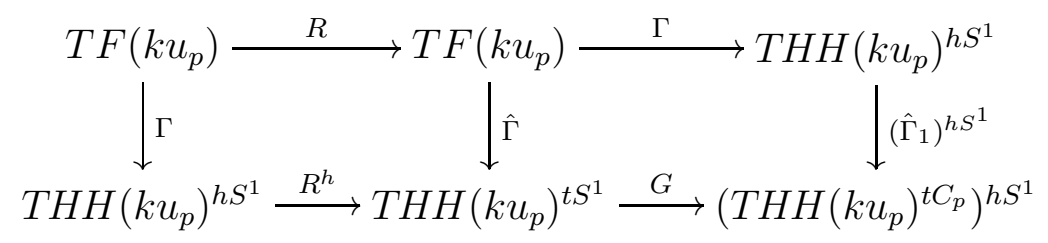

displayed in [3, page 27], and with $G$ a $V(1)$-equivalence. By the argument in [3, Lemma 7.5], we know that on $V(1)_{*} T F\left(k u_{p}\right)$ the profinite topology coincides with the topology induced by the spectral sequence filtration of $V(1)_{*} T H H\left(k u_{p}\right)^{h S^{1}}$ via $\Gamma_{*}$, and that the restriction map

$$
R_{*}: V(1)_{*} T F\left(k u_{p}\right) \rightarrow V(1)_{*} T F\left(k u_{p}\right)
$$

is continuous in degrees larger than $2 p-2$. In this range of degrees, we will identify $V(1)_{*} T F\left(k u_{p}\right)$ with $V(1)_{*} T H H\left(k u_{p}\right)^{h S^{1}}$ via the homeomorphism $\Gamma_{*}$. Under this identification $R_{*}$ corresponds to $\left(\Gamma_{*} \hat{\Gamma}_{*}^{-1}\right) R_{*}^{h}$, and we first describe $R_{*}^{h}$ and $\Gamma_{*} \hat{\Gamma}_{*}^{-1}$ separately.

Lemma 7.1. In total degrees larger than $2 p-2$, the morphism

$$
\left(R^{h}\right)^{\infty}: E^{\infty}\left(S^{1}\right) \rightarrow \hat{E}^{\infty}\left(S^{1}\right)
$$

has the following properties.

(a) It maps $E\left(\lambda_{1}, a_{1}\right) \otimes P_{p-1}(b) \otimes P(t \mu)$ isomorphically to the summand with same name;

(b) It maps $E\left(\lambda_{1}\right) \otimes P_{r(k)}(t \mu) \otimes \mathbb{F}_{p}\left\{\lambda_{2} \mu^{-d p^{k-1}}\right\}$ onto

$$
E\left(\lambda_{1}\right) \otimes P_{r(k-2)}(t \mu) \otimes \mathbb{F}_{p}\left\{\lambda_{2} t^{d p^{k-1}}\right\}
$$

and $E\left(\lambda_{1}\right) \otimes P_{p-2}(b) \otimes P_{r(k)+1}(t \mu) \otimes \mathbb{F}_{p}\left\{a_{1} \mu^{-d p^{k-1}}\right\}$ onto

$$
E\left(\lambda_{1}\right) \otimes P_{p-2}(b) \otimes P_{r(k-2)+1}(t \mu) \otimes \mathbb{F}_{p}\left\{a_{1} t^{d p^{k-1}}\right\}
$$

for $k \geqslant 2$ even and $0<d<p$;

(c) It maps $E\left(a_{1}\right) \otimes P_{p-1}(b) \otimes P_{r(k)}(t \mu) \otimes \mathbb{F}_{p}\left\{\lambda_{1} \mu^{-d p^{k-1}}\right\}$ onto

$$
E\left(a_{1}\right) \otimes P_{p-1}(b) \otimes P_{r(k-2)}(t \mu) \otimes \mathbb{F}_{p}\left\{\lambda_{1} t^{d p^{k-1}}\right\}
$$

for $k \geqslant 3$ odd and $0<d<p$;

(d) It maps the remaining summands to zero.

Proof. This follows from the description of $\left(R^{h}\right)^{2}$, see (6.2).

Lemma 7.2. In degrees larger then $2 p-2$, the homomorphism $\Gamma_{*} \hat{\Gamma}_{*}^{-1}$ maps

(a) the classes in $V(1)_{*} T H H\left(k u_{p}\right)^{t S^{1}}$ represented in $\hat{E}^{\infty}\left(S^{1}\right)$ by

$$
\lambda_{1}^{\epsilon_{1}} a_{1}^{\epsilon_{2}} b^{k}(t \mu)^{m} t^{i}
$$

for $v_{p}(i) \neq 1, \epsilon_{1}$ and $\epsilon_{2} \in\{0,1\}, 0 \leqslant k \leqslant p-2$ and $m \geqslant 0$, to classes in $V(1)_{*} T H H\left(k u_{p}\right)^{h S^{1}}$ represented in $E^{\infty}\left(S^{1}\right)$ by

$$
\lambda_{1}^{\epsilon_{1}} a_{1}^{\epsilon_{2}} b^{k}(t \mu)^{m} \mu^{j}
$$

with $i+p^{2} j=0$, up to multiplication with a unit in $\mathbb{F}_{p}$; 
(b) the classes in $V(1)_{*} T H H\left(k u_{p}\right)^{t S^{1}}$ represented in $\hat{E}^{\infty}\left(S^{1}\right)$ by

$$
\lambda_{1}^{\epsilon_{1}} b^{k} a_{1} t^{i}
$$

for $v_{p}(i)=1, \epsilon_{1} \in\{0,1\}$ and $0 \leqslant k \leqslant p-3$, to classes in $V(1)_{*} T H H\left(k u_{p}\right)^{h S^{1}}$ represented in $E^{\infty}\left(S^{1}\right)$ by

$$
\lambda_{1}^{\epsilon_{1}} b^{k} \mu^{l} a_{j}
$$

with $i=(1-j) p-l p^{2}$ for $0 \leqslant j \leqslant p-1$ such that $j \neq 1$, up to multiplication with a unit in $\mathbb{F}_{p}$.

Proof. The proof is similar to the proof of [3, Proposition 7.4], and we omit it.

Definition 7.3. We recall from [3, Theorem 9.1] that there are classes $\lambda_{1} t^{p-1}, \lambda_{1}$ and $\lambda_{2}$ in $V(1)_{*} K\left(\ell_{p}\right) \subset V(1)_{*} K\left(k u_{p}\right)$, of degree $1,2 p-1$ and $2 p^{2}-1$, respectively. We denote by

$$
\widetilde{\lambda_{1} t^{p-1}}, \quad \tilde{\lambda}_{1} \text { and } \tilde{\lambda}_{2}
$$

their image in $V(1)_{*} T F\left(k u_{p}\right)$ under $\operatorname{tr}_{F *}$. The latter classes are represented by

$$
\lambda_{1} t^{p-1}=(t \mu)^{p-1} \cdot \lambda_{1} \mu^{1-p}, \lambda_{1} \text { and } \lambda_{2}
$$

in $E^{\infty}\left(S^{1}\right)$, respectively, see [3, Theorem 8.4]. We further denote by $b$ and $v_{2}$ the image in $V(1)_{*} T F\left(k u_{p}\right)$ under $\operatorname{tr}_{F_{*}}$ of the classes with same name in $V(1)_{*} K\left(k u_{p}\right)$. These classes are represented by $b_{1}$ and $t \mu$ in $E^{\infty}\left(S^{1}\right)$, respectively, see Lemma 4.4 and [3, Proposition 4.8].

Lemma 7.4. There exists a unique class $\tilde{a}_{1} \in V(1)_{2 p+3} T F\left(k u_{p}\right)$ with the following two properties:

(a) $\tilde{a}_{1}$ has $\delta$-weight 1 and $b^{p-2} \tilde{a}_{1}=\tilde{\lambda}_{2}$,

(b) $R_{*}\left(\tilde{a}_{1}\right)=\tilde{a}_{1}$.

Moreover, this class $\tilde{a}_{1}$ is represented by $a_{1}$ in $E^{\infty}\left(S^{1}\right)$.

Proof. For $i=0$ or 1 , let us denote by $T_{*}^{(i)}$ and $\operatorname{ker}(R-1)_{*}^{(i)}$ the summand of $\delta$-weight $i$ of $V(1)_{*} T F\left(k u_{p}\right)$ and $\operatorname{ker}(R-1)_{*} \subset V(1)_{*} T F\left(k u_{p}\right)$, respectively. We make the following claims :

(1) The homomorphism given by multiplication with $b^{p-2}$ on $T_{2 p+3}^{(1)}$ fits in a short exact sequence

$$
0 \rightarrow \mathbb{F}_{p}\{z\} \rightarrow T_{2 p+3}^{(1)} \stackrel{b^{p-2}}{\longrightarrow} T_{2 p^{2}-1}^{(0)} \rightarrow 0
$$

where the class $z$ is represented by $b_{1} \cdot(t \mu)^{p-1} \cdot \lambda_{1} \mu^{1-p}$ in $E^{\infty}\left(S^{1}\right)$;

(2) The class $z$ does not belong to $\operatorname{ker}(R-1)_{*}$.

Using these claims, it is easy to deduce that multiplication with $b^{p-2}$ restricts to an isomorphism

$$
\operatorname{ker}(R-1)_{2 p+3}^{(1)} \stackrel{\cong}{\longrightarrow} \operatorname{ker}(R-1)_{2 p^{2}-1}^{(0)} .
$$

We have $\tilde{\lambda}_{2} \in \operatorname{ker}(R-1)_{2 p^{2}-1}^{(0)}$ since $\tilde{\lambda}_{2}$ has $\delta$-weight 0 and is in the image of $\operatorname{tr}_{F *}$. Therefore, there is a unique pre-image $\tilde{a}_{1} \in \operatorname{ker}(R-1)_{2 p+3}^{(1)}$ of $\tilde{\lambda}_{2} \in \operatorname{ker}(R-1)_{2 p^{2}-1}^{(0)}$, or, in other words, there is a unique class $\tilde{a}_{1} \in V(1)_{2 p+3} T F\left(k u_{p}\right)$ with properties (a) and (b). Moreover, $\tilde{\lambda}_{2}$ is represented in $E^{\infty}\left(S^{1}\right)$ in filtration zero by $\lambda_{2}=b_{1}^{p-2} a_{1}$, and we deduce that $\tilde{a}_{1}$ must be represented in filtration zero by $a_{1}$. Thus this lemma follows from claims (1) and (2), which we now prove. 
First, notice that the group $T_{*}^{(i)}$ inherits via $\Gamma_{*}$ the spectral sequence filtration of $V(1)_{*} T H H\left(k u_{p}\right)^{h S^{1}}$. Denoting by $E^{\infty}\left(S^{1}\right)_{*}^{(i)}$ its associated graded, we know from Lemma 6.13 that

$$
\begin{aligned}
E^{\infty}\left(S^{1}\right)_{2 p+3}^{(1)} & =\mathbb{F}_{p}\left\{a_{1}, b_{1} \cdot x_{n} \mid n \geqslant 0\right\} \text { and } \\
E^{\infty}\left(S^{1}\right)_{2 p^{2}-1}^{(0)} & =\mathbb{F}_{p}\left\{\lambda_{2}, t \mu \cdot x_{n} \mid n \geqslant 1\right\},
\end{aligned}
$$

where $x_{n}=(t \mu)^{r(2 n+1)-r(2 n)-1} \cdot \lambda_{1} \mu^{(1-p) p^{2 n}}$.

Next, the relation $b^{\prime p}+v_{2} b^{\prime}=0$ in $V(1)_{*} K(\mathbb{Z}, 3)$, established in Proposition 2.7, maps under $\operatorname{tr}_{F *} \phi_{*}$ to the relation $b^{p}+v_{2} b=0$ in $T_{*}^{(1)}$. The class $v_{2} b$ in $T_{*}^{(1)}$ is represented by the non-zero class $t \mu \cdot b_{1}$ in $E^{\infty}\left(S^{1}\right)$ in filtration -2 , and we deduce that $b^{p-1} \in T_{*}^{(0)}$ must be represented by $-t \mu$ in $E^{\infty}\left(S^{1}\right)$. It follows that if a class $x \in T_{2 p+3}^{(1)}$ is represented by $b_{1} \cdot x_{n}$, then $b^{p-2} x$ is represented by $-t \mu \cdot x_{n}$ in 2 filtration degrees lower. Using a coarser filtration that ignores this shift, and considering our formulas for $E^{\infty}\left(S^{1}\right)_{2 p+3}^{(1)}$ and $E^{\infty}\left(S^{1}\right)_{2 p^{2}-1}^{(0)}$ given above, we deduce claim (1) from the corresponding claim for the associated graded, with $z$ represented by $b_{1} \cdot x_{0}$.

To prove claim (2), we notice that if a class $y \in T_{2 p+3}^{(1)}$ is represented by $b_{1} \cdot x_{n}$ with $n \geqslant 1$, then $R_{*}(y)$ will be represented by $b_{1} \cdot x_{n-1}$ in higher filtration, up to some non-zero scalar multiple: this follows directly from Lemmas 7.1 and 7.2 . In particular, $R_{*}(y) \neq y$. This implies the following claim:

(3) The group $\operatorname{ker}(R-1)_{2 p+3}^{(1)}$ contains at most one class represented by $b_{1} \cdot x_{0}$.

Now consider the class $\tilde{x}_{0}=\widetilde{\lambda_{1} t^{p-1}} \in T_{1}^{(0)}$ given in Definition [7.3. By definition, this class lies in $\operatorname{ker}(R-1)_{1}^{(0)}$ and is represented by $x_{0}$. We also claim that

(4) The class $b \tilde{x}_{0} \in \operatorname{ker}(R-1)_{2 p+3}^{(1)}$ is not annihilated by $b^{p-2}$.

Since $b \tilde{x}_{0}$ is represented by $b_{1} \cdot x_{0}$, claim (2) follows from claims (3) and (4).

Finally, to prove claim (4), we recall from [3, Theorem 8.2] that the class $v_{2} \tilde{x}_{0} \in$ $\operatorname{ker}(R-1)_{2 p^{2}-1}^{(0)}$ is non-zero, and must be represented, in filtration degree lower then $-2 p+2$, by a class in

$$
\mathbb{F}_{p}\left\{t \mu \cdot x_{n} \mid n \geqslant 1\right\}
$$

None of these classes is annihilated by $b_{1}$. Therefore $b v_{2} \tilde{x}_{0}=-b^{p} \tilde{x}_{0}$ is non-zero, and we deduce that $b \tilde{x}_{0} \in \operatorname{ker}(R-1)_{2 p+3}^{(1)}$ is not annihilated by $b^{p-2}$.

Remark 7.5. The lemma above implies that the class $a_{1} \in V(1)_{*} T H H\left(k u_{p}\right)$ has a lift $a_{1} \in V(1)_{*} K\left(k u_{p}\right)$ under the trace, with $b^{p-2} a_{1}=\lambda_{2}$, see Theorem 8.1. It would be nice to have a more direct construction of such a lift. In fact, we conjecture that $a_{1} \in$ $V(1)_{*} K\left(k u_{p}\right)$ decomposes as $b d$, where $d \in V(1)_{1} K\left(K U_{p}\right)$ is a unit class, when mapped into $V(1)_{*} K\left(K U_{p}\right)$, see the discussion preceding Theorem 8.3 below. 
Definition 7.6. We consider the following subgroups of $E^{\infty}\left(S^{1}\right)$ :

$$
\begin{aligned}
& A= E\left(\lambda_{1}, a_{1}\right) \otimes P_{p-1}\left(b_{1}\right) \otimes P(t \mu) \\
& B_{0}= E\left(\lambda_{1}\right) \otimes P_{p-2}\left(b_{1}\right) \otimes \mathbb{F}_{p}\left\{\mu^{-1} a_{i}, a_{0} \mid 2 \leqslant i \leqslant p-1\right\} \\
& B_{k}=\left(E\left(\lambda_{1}\right) \otimes P_{p-2}\left(b_{1}\right) \otimes \bigoplus_{0<d<p}\left(P_{r(k)-d p^{k-1}+1}(t \mu) \otimes \mathbb{F}_{p}\left\{a_{1} t^{d p^{k-1}}\right\}\right)\right) \\
& \oplus\left(E\left(\lambda_{1}\right) \otimes \bigoplus_{0<d<p} P_{r(k)-d p^{k-1}}(t \mu) \otimes \mathbb{F}_{p}\left\{\lambda_{2} t^{d p^{k-1}}\right\}\right) \text { for } k \geqslant 2 \text { even, } \\
& B_{k}=E\left(a_{1}\right) \otimes P_{p-1}\left(b_{1}\right) \otimes \bigoplus_{0<d<p}\left(P_{r(k)-d p^{k-1}}(t \mu) \otimes \mathbb{F}_{p}\left\{\lambda_{1} t^{d p^{k-1}}\right\}\right) \text { for } k \geqslant 1 \text { odd, }
\end{aligned}
$$

and we let $C$ be the span of the remaining monomials in $E^{\infty}\left(S^{1}\right)$. We then have a direct sum decomposition $E^{\infty}\left(S^{1}\right)=A \oplus B \oplus C$, with $B=\bigoplus_{k \geqslant 0} B_{k}$.

Lemma 7.7. In dimensions larger than $2 p-2$ there are closed subgroups $\tilde{A}, \tilde{B}_{k}$ and $\tilde{C}$ in $V(1)_{*} T F\left(k u_{p}\right)$, represented by $A, B_{k}$ and $C$ in $E^{\infty}\left(S^{1}\right)$ respectively, such that

(a) $R_{*}$ restricts to the identity on $\tilde{A}$,

(b) $R_{*}$ maps $\tilde{B}_{k+2}$ onto $\tilde{B}_{k}$ for $k \geqslant 0$,

(c) $R_{*}$ maps $\tilde{B}_{0}, \tilde{B}_{1}$ and $\tilde{C}$ to zero.

In these degrees $V(1)_{*} T F\left(k u_{p}\right) \cong \tilde{A} \oplus \tilde{B} \oplus \tilde{C}$, where $\tilde{B}=\prod_{k \geqslant 0} \tilde{B}_{k}$.

Proof. On the associated graded $E^{\infty}\left(S^{1}\right)$, the homomorphism $\left(\Gamma_{*} \hat{\Gamma}_{*}^{-1}\right) R_{*}^{h}$ has been described in Lemmas 7.1 and 7.2 , and maps $A$ isomorphically to itself, $B_{k+2}$ onto $B_{k}$ for $k \geqslant 0$, and $B_{0}, B_{1}$ and $C$ to zero. It remains to find closed lifts of these groups in $V(1)_{*} T F\left(k u_{p}\right)$ with desired properties. We take $\tilde{A}$ to be the (closed) subalgebra of $V(1)_{*} T F\left(k u_{p}\right)$ generated by $\tilde{\lambda}_{1}, \tilde{a}_{1}, b$ and $v_{2}$. Then $\tilde{A}$ lifts $A$, by definition of its algebra generators and by the fact, proved above, that $b^{p-1}$ is represented by $-t \mu$ in $E^{\infty}\left(S^{1}\right)$. Also, $\tilde{\lambda}_{1}, b$ and $v_{2}$ are fixed under $R_{*}$, since they are in the image of $\operatorname{tr}_{F *}$, and $\tilde{a}_{1}$ is fixed by definition. To construct $\tilde{B}_{k}$ for $k \geqslant 0$ and $\tilde{C}$, we follow the procedure given in [3, Theorem 7.7].

Definition 7.8. We denote $b \in V(1)_{2 p+2} T C\left(k u_{p}\right)$ the image of the higher Bott element $b$, defined in 3.2, under the cyclotomic trace map

$$
(\operatorname{trc})_{*}: V(1)_{*} K\left(k u_{p}\right) \rightarrow V(1)_{*} T C\left(k u_{p}\right) .
$$

Theorem 7.9. The class $b \in V(1)_{2 p+2} T C\left(k u_{p}\right)$ satisfies the relation

$$
b^{p-1}=-v_{2} .
$$

There is an isomorphism of $P(b)$-modules

$$
\begin{aligned}
V(1)_{*} T C\left(k u_{p}\right) \cong & P(b) \otimes E\left(\partial, \lambda_{1}, a_{1}\right) \\
& \oplus P(b) \otimes E\left(a_{1}\right) \otimes \mathbb{F}_{p}\left\{t^{d} \lambda_{1} \mid 0<d<p\right\} \\
& \oplus P(b) \otimes E\left(\lambda_{1}\right) \otimes \mathbb{F}_{p}\left\{u^{i} a_{0}, t^{p^{2}-p} \lambda_{2} \mid 0 \leqslant i<p-2\right\},
\end{aligned}
$$

where the degree of the classes is $|\partial|=-1,\left|\lambda_{1}\right|=2 p-1,\left|a_{1}\right|=2 p+3,\left|u^{i} a_{0}\right|=2 i+3$, $\left|\lambda_{2}\right|=2 p^{2}-1$ and $|t|=-2$. 
Proof. Recall that $T C\left(k u_{p}\right)$ is defined as the homotopy fiber of the map

$$
R-1: T F\left(k u_{p}\right) \rightarrow T F\left(k u_{p}\right) .
$$

In $V(1)$-homotopy, it gives a short exact sequence of $P\left(v_{2}\right)$-modules

$$
0 \rightarrow \Sigma^{-1} \operatorname{cok}(R-1)_{*} \rightarrow V(1)_{*} T C\left(k u_{p}\right) \rightarrow \operatorname{ker}(R-1)_{*} \rightarrow 0 .
$$

We have isomorphisms of $P\left(v_{2}\right)$-modules

$$
\begin{aligned}
\Sigma^{-1} \operatorname{cok}(R-1)_{*} & \cong \Sigma^{-1} \tilde{A} \\
\operatorname{ker}(R-1)_{*} & \cong \tilde{A} \oplus \lim _{k \geqslant 0 \text { even }} \tilde{B}_{k} \oplus \lim _{k \geqslant 1 \text { odd }} \tilde{B}_{k} .
\end{aligned}
$$

Indeed, $R_{*}-1$ maps each factor of the decomposition $V(1)_{*} T F\left(k u_{p}\right) \cong \tilde{A} \oplus \tilde{B} \oplus \tilde{C}$ to itself. It restricts to zero on $\tilde{A}$ and to the identity on $\tilde{C}$. We have a short exact sequence

$$
0 \rightarrow \lim _{k \geqslant 0 \text { even }} \tilde{B}_{k} \rightarrow \prod_{k \geqslant 0 \text { even }} \tilde{B}_{k} \stackrel{R_{*}-1}{\longrightarrow} \prod_{k \geqslant 0 \text { even }} \tilde{B}_{k} \rightarrow \lim _{k \geqslant 0 \text { even }}^{1} \tilde{B}_{k} \rightarrow 0
$$

and similarly for the $\tilde{B}_{k}$ with $k$ odd. Here the limits are taken over the sequential system of maps $R_{*}: \tilde{B}_{k+2} \rightarrow \tilde{B}_{k}$ for $k \geqslant 0$ even or $k \geqslant 1$ odd. Since these maps are surjective, the $\lim ^{1}$-terms are trivial. This proves our claims on $\Sigma^{-1} \operatorname{cok}(R-1)_{*}$ and $\operatorname{ker}(R-1)_{*}$ in (17.2).

For $k \geqslant 1$ odd, the group $\tilde{B}_{k}$ is isomorphic as a $P\left(v_{2}\right)$-module to a sum of $2(p-1)^{2}$ cyclic $P\left(v_{2}\right)$-modules

$$
\tilde{B}^{k} \cong E\left(a_{1}\right) \otimes P_{r(k)}\left(v_{2}\right) \otimes P_{p-1}(b) \otimes \mathbb{F}_{p}\left\{\lambda_{1} t^{d p^{k-1}} \mid 0<d<p\right\} .
$$

The map $R_{*}$ respects this decomposition into cyclic $P\left(v_{2}\right)$-modules. Since the height of these modules grows to infinity with $k$, we deduce from the surjectivity of $R_{*}$ that $\lim _{k \geqslant 1}$ odd $\tilde{B}_{k}$ is a sum of $2(p-1)^{2}$ free cyclic $P\left(v_{2}\right)$-modules, given by an isomorphism

$$
\lim _{k \geqslant 1 \text { odd }} \tilde{B}_{k} \cong E\left(a_{1}\right) \otimes P\left(v_{2}\right) \otimes P_{p-1}(b) \otimes \mathbb{F}_{p}\left\{\lambda_{1} t^{d} \mid 0<d<p\right\} .
$$

Similarly, for $k \geqslant 2$ even, $\tilde{B}_{k}$ is isomorphic to a sum of $2(p-1)^{2}$ cyclic $P\left(v_{2}\right)$-modules of height growing with $k$, and passing to the limit we have an isomorphism of $P\left(v_{2}\right)$-modules

$$
\lim _{k \geqslant 0 \text { even }} \tilde{B}_{k} \cong E\left(\lambda_{1}\right) \otimes P\left(v_{2}\right) \otimes P_{p-1}(b) \otimes \mathbb{F}_{p}\left\{a_{1} t^{d p} \mid 0<d<p\right\} .
$$

Thus $\operatorname{ker}(R-1)_{*}$ is a free $P\left(v_{2}\right)$-module, and the exact sequence (17.1) splits. We have an isomorphism of $P\left(v_{2}\right)$-modules

$$
\begin{aligned}
V(1)_{*} T C\left(k u_{p}\right) \cong & P\left(v_{2}\right) \otimes P_{p-1}(b) \otimes E\left(\partial, \lambda_{1}, a_{1}\right) \\
& \oplus P\left(v_{2}\right) \otimes P_{p-1}(b) \otimes E\left(a_{1}\right) \otimes \mathbb{F}_{p}\left\{\lambda_{1} t^{d} \mid 0<d<p\right\} \\
& \oplus P\left(v_{2}\right) \otimes P_{p-1}(b) \otimes E\left(\lambda_{1}\right) \otimes \mathbb{F}_{p}\left\{a_{1} t^{p d} \mid 0<d<p\right\}
\end{aligned}
$$

in degrees larger than $2 p-2$, where the summand

$$
P\left(v_{2}\right) \otimes P_{p-1}(b) \otimes E\left(\lambda_{1}, a_{1}\right) \otimes \mathbb{F}_{p}\{\partial\}
$$

is the $\operatorname{group} \operatorname{cok}(R-1)_{*} \cong \Sigma^{-1} \tilde{A}$.

We now show that the relation

$$
b^{p-1}=-v_{2}
$$


holds in $V(1)_{*} T C\left(k u_{p}\right)$. We recall from Proposition 2.7 that the class $b^{p-1}+v_{2}$ in $V(1)_{2 p^{2}-2} K(\mathbb{Z}, 3)$ is annihilated by $b^{\prime}$. This class maps by $\operatorname{trc}_{*} \phi_{*}$ to the class

$$
b^{p-1}+v_{2} \in V(1)_{2 p^{2}-2} T C\left(k u_{p}\right),
$$

which is therefore annihilated by $b$. Thus it suffices to show that zero is the only class in $V(1)_{2 p^{2}-2} T C\left(k u_{p}\right)$ that is annihilated by $b$. We consider the short exact sequence

$$
0 \rightarrow \operatorname{cok}(R-1)_{2 p^{2}-1} \rightarrow V(1)_{2 p^{2}-2} T C\left(k u_{p}\right) \rightarrow \operatorname{ker}(R-1)_{2 p^{2}-2} \rightarrow 0
$$

given in (7.1) above. Here

$$
\operatorname{ker}(R-1)_{*} \subset V(1)_{*} T F\left(k u_{p}\right)
$$

inherits via $\Gamma_{*}$ the spectral sequence filtration of $V(1)_{*} T H H\left(k u_{p}\right)^{h S^{1}}$. By (17.3), this filtration gives the short exact sequence

$$
0 \rightarrow \mathbb{F}_{p}\left\{b^{p-2} \cdot \lambda_{1} \cdot a_{1} t^{p}\right\} \rightarrow \operatorname{ker}(R-1)_{2 p^{2}-2} \rightarrow \mathbb{F}_{p}\left\{\overline{v_{2}}\right\} \rightarrow 0
$$

in dimension $2 p^{2}-2$, while in dimension $2 p^{2}+2 p$ it gives the short exact sequence

$$
0 \rightarrow \mathbb{F}_{p}\left\{v_{2} \cdot \lambda_{1} \cdot a_{1} t^{p}\right\} \rightarrow \operatorname{ker}(R-1)_{2 p^{2}+2 p} \rightarrow \mathbb{F}_{p}\left\{\overline{b \cdot v_{2}}\right\} \rightarrow 0 .
$$

Here $\overline{v_{2}}$ and $\overline{b \cdot v_{2}}$ are represented by $t \mu$ and and $b_{1} \cdot t \mu$ in $E^{\infty}\left(S^{1}\right)$, respectively. Multiplication with $b$ is compatible with the filtration, and maps the former sequence to the latter one. First, notice that the class $\overline{v_{2}}$ maps to a non-zero class in $\mathbb{F}_{p}\left\{\overline{b \cdot v_{2}}\right\}$, since $b \cdot \overline{v_{2}}$ is represented by $b_{1} \cdot t \mu$ in $E^{\infty}\left(S^{1}\right)$. Next, the relation $b^{p}=-b v_{2}$ in $\operatorname{ker}(R-1)_{*}$ implies

$$
b^{p} \cdot \lambda_{1} \cdot a_{1} t^{p}=-v_{2} \cdot b \cdot \lambda_{1} \cdot a_{1} t^{p},
$$

which is non-zero by (17.3). A fortiori $b^{p-1} \cdot \lambda_{1} \cdot a_{1} t^{p} \in \mathbb{F}_{p}\left\{v_{2} \cdot \lambda_{1} \cdot a_{1} t^{p}\right\}$ is not zero either. Thus $\operatorname{ker}(R-1)_{2 p^{2}-2}$ contains no non-zero class annihilated by $b$, and we deduce that

$$
b^{p-1}+v_{2} \in \partial\left(\operatorname{cok}(R-1)_{2 p^{2}-1}\right)=\mathbb{F}_{p}\left\{b^{p-2} \cdot a_{1} \cdot \partial\right\} .
$$

However the class $b^{p-2} \cdot a_{1} \cdot \partial$ is not annihilated by $b$, since by (7.3) we know that $b^{p} \cdot a_{1} \cdot \partial=-v_{2} \cdot b \cdot a_{1} \cdot \partial$ is non-zero. This proves that $b^{p-1}+v_{2}$ must be zero.

In particular $b$ is not a nilpotent class, and we have an isomorphism of $P(b)$-modules

$$
\begin{aligned}
V(1)_{*} T C\left(k u_{p}\right) \cong & P(b) \otimes E\left(\partial, \lambda_{1}, a_{1}\right) \\
& \oplus P(b) \otimes E\left(a_{1}\right) \otimes \mathbb{F}_{p}\left\{t^{d} \lambda_{1} \mid 0<d<p\right\} \\
& \oplus P(b) \otimes E\left(\lambda_{1}\right) \otimes \mathbb{F}_{p}\left\{a_{1} t^{p d} \mid 0<d<p\right\}
\end{aligned}
$$

in degrees larger than $2 p-2$. This proves that our formula for $V(1)_{*} T C\left(k u_{p}\right)$ is correct in dimensions greater than $2 p-2$. Let us define $M$ and $N$ as

$$
M=\bigoplus_{-1 \leqslant n \leqslant 2 p-2} V(1)_{n} T C\left(k u_{p}\right) \text { and } N=\bigoplus_{n \geqslant 2 p-1} V(1)_{n} T C\left(k u_{p}\right) .
$$

We just argued that $N$ is a free $P(b)$-module. We know by (5.3) that there is an isomorphism

$$
M \cong \mathbb{F}_{p}\left\{\partial, 1, u^{i} a_{0}, \lambda_{1} t^{d}, \partial \lambda_{1} \mid 0 \leqslant i \leqslant p-3,1 \leqslant d \leqslant p-1\right\}
$$

of $\mathbb{F}_{p}$-modules. This proves that the formula for $V(1)_{*} T C\left(k u_{p}\right)$ in Theorem 7.9 holds as an isomorphism of $\mathbb{F}_{p}$-modules. It only remains to show that for any non-zero class $m \in M$, we have $b m \neq 0$ in $V(1)_{*} T C\left(k u_{p}\right)$. By comparison with $V(1)_{*} T C\left(\ell_{p}\right)$ or with $V(1)_{*} T H H\left(k u_{p}\right)^{h S^{1}}$, we know that either $m \lambda_{1}$ or $m v_{2}$ is non-zero. These products lie in $N$ for degree reasons, so are not $b$-torsion classes. Therefore $m$ is not a $b$-torsion class either. 


\section{Algebraic K-Theory}

Theorem 8.1. There is an isomorphism of $P(b)$-modules

$$
\begin{aligned}
V(1)_{*} K\left(k u_{p}\right) \cong & P(b) \otimes E\left(\lambda_{1}, a_{1}\right) \oplus P(b) \otimes \mathbb{F}_{p}\left\{\partial \lambda_{1}, \partial b, \partial a_{1}, \partial \lambda_{1} a_{1}\right\} \\
& \oplus P(b) \otimes E\left(a_{1}\right) \otimes \mathbb{F}_{p}\left\{t^{d} \lambda_{1} \mid 0<d<p\right\} \\
& \oplus P(b) \otimes E\left(\lambda_{1}\right) \otimes \mathbb{F}_{p}\left\{\sigma_{n}, \lambda_{2} t^{p^{2}-p} \mid 1 \leqslant n \leqslant p-2\right\} \\
& \oplus \mathbb{F}_{p}\{s\},
\end{aligned}
$$

with $b^{p-1}=-v_{2}$. The degree of the generators is given by $|\partial|=-1,\left|\lambda_{1}\right|=2 p-1$, $\left|a_{1}\right|=2 p+3,\left|\sigma_{n}\right|=2 n+1,|t|=-2,\left|\lambda_{2}\right|=2 p^{2}-1$ and $|s|=2 p-3$. The classes $1, \sigma_{n}, \lambda_{1}, b$ and $a_{1}$ map under the trace to $1, u^{n-1} a_{0}, \lambda_{1}, b_{1}$ and $a_{1}$ in $V(1)_{*} \operatorname{THH}\left(k u_{p}\right)$, respectively, and the other given $P(b)$-module generators map to zero.

Proof. There is a cofibre sequence of spectra [22]

$$
K\left(k u_{p}\right)_{p} \rightarrow T C\left(k u_{p}\right) \rightarrow \Sigma^{-1} H \mathbb{Z}_{p} \rightarrow \Sigma K\left(k u_{p}\right)_{p} .
$$

We have an isomorphism $V(1)_{*} \Sigma^{-1} H \mathbb{Z}_{p} \cong \mathbb{F}_{p}\{\partial, \epsilon\}$ with a primary $v_{1}$ Bockstein $\beta_{1,1}(\epsilon)=$ $\partial$. Here $\partial$ is the image of the class $\partial \in V(1)_{-1} T C\left(k u_{p}\right)$, while $\epsilon$ maps by the connecting homomorphism to a class $s \in V(1)_{2 p-3} K\left(k u_{p}\right)$. These facts, together with Theorem 7.9 , allow us to establish our formula for $V(1)_{*} K\left(k u_{p}\right)$. The statement on the trace follows from the definition of the given $P(b)$-module generators.

The following corollary is a restatement of Proposition 1.2 part (b) of the introduction.

Corollary 8.2. There is a short exact sequence of $P(b)$-modules

$$
0 \rightarrow K \rightarrow P(b) \otimes_{P\left(v_{2}\right)} V(1)_{*} K\left(\ell_{p}\right) \stackrel{\mu}{\longrightarrow} K\left(k u_{p}\right) \rightarrow Q \rightarrow 0
$$

where $K$ and $Q$ are finite (and hence torsion) $P(b)$-modules given by

$$
\begin{aligned}
K= & \mathbb{F}_{p}\left\{b^{k} a \mid 1 \leqslant k \leqslant p-2\right\}, \text { and } \\
Q= & P_{p-2}(b) \otimes \mathbb{F}_{p}\left\{\partial b, \partial a_{1}, a_{1}, \partial \lambda_{1} a_{1}, \lambda_{1} a_{1}\right\} \\
& \oplus P_{p-2}(b) \otimes \mathbb{F}_{p}\left\{a_{1} \lambda_{1} t^{d} \mid 0<d<p\right\} \\
& \oplus E\left(\lambda_{1}\right) \otimes \mathbb{F}_{p}\left\{\sigma_{n} b^{i_{n}} \mid 1 \leqslant n \leqslant p-2,0 \leqslant i_{n} \leqslant p-2-n\right\} .
\end{aligned}
$$

Here $a \in V(1)_{2 p-3} K\left(\ell_{p}\right)$ is the class annihilated by $v_{2}$ and mapping to $s$. In particular we have an isomorphism $P\left(b, b^{-1}\right)$-algebras

$$
P\left(b, b^{-1}\right) \otimes_{P\left(v_{2}\right)} V(1)_{*} K\left(\ell_{p}\right) \cong V(1)_{*} K\left(k u_{p}\right)\left[b^{-1}\right] .
$$

Proof. This follows from the formulas for $V(1)_{*} K\left(\ell_{p}\right)$ and for $V(1)_{*} K\left(k u_{p}\right)$ given in [3, Theorem 9.1] and Theorem 8.1, and the fact that $V(1)_{*} K\left(\ell_{p}\right)$ includes as the summand of $\delta$-weight zero in $V(1)_{*} K\left(k u_{p}\right)$, see [1, Theorem 10.2]. Notice that for $1 \leqslant d \leqslant p-2$ the class $\lambda_{2} t^{d p} \in V(1)_{2 p^{2}-p d-1} K\left(\ell_{p}\right)$ maps to $\sigma_{d} b^{p-1-d}$, up to a non-zero scalar multiple.

Blumberg and Mandell [8] have proved a conjecture of John Rognes that there is a localization cofibre sequence

$$
K\left(\mathbb{Z}_{p}\right) \stackrel{\tau}{\longrightarrow} K\left(k u_{p}\right) \stackrel{j}{\longrightarrow} K\left(K U_{p}\right) \rightarrow \Sigma K\left(\mathbb{Z}_{p}\right),
$$

relating the algebraic $K$-theory of $k u_{p}$, of its localization $K U_{p}=k u_{p}\left[u^{-1}\right]$ (i.e. periodic $K$-theory), and of its $\bmod (u)$ reduction $H \mathbb{Z}_{p}$. The $V(1)$-homotopy of $K\left(\mathbb{Z}_{p}\right)$ and $K\left(k u_{p}\right)$ is known, but we need to compute also the transfer map $\tau_{*}$ and solve a $P(b)$-module 
extension if we seek a decent description of $V(1)_{*} K\left(K U_{p}\right)$. Let us therefore assume that this localization sequence maps via trace maps to a corresponding localization sequence in topological Hochschild homology, building a homotopy commutative diagram of horizontal fibre sequences

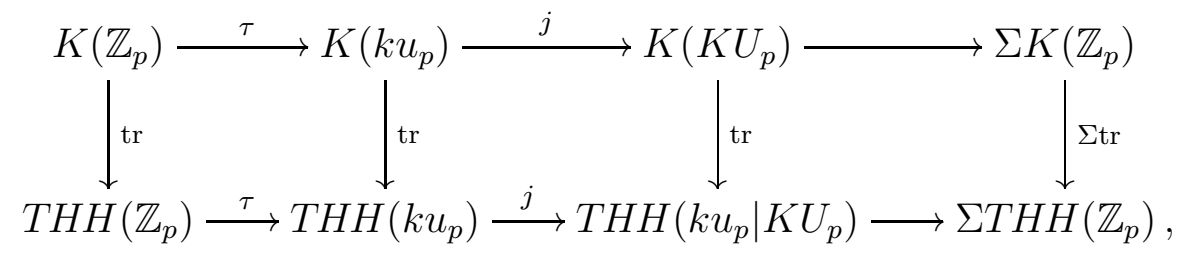

as conjectured by Lars Hesselholt, compare with Remark 8.4 below. The $V(1)$-homotopy of the bottom line was described in [1, §10]. The $V(1)$-homotopy groups of $K\left(\mathbb{Z}_{p}\right)$ are given by an isomorphism 22

$$
V(1)_{*} K\left(\mathbb{Z}_{p}\right) \cong E\left(\lambda_{1}\right) \oplus \mathbb{F}_{p}\left\{\partial v_{1}, \partial \lambda_{1}\right\} \oplus \mathbb{F}_{p}\left\{\lambda_{1} t^{d} \mid 0<d<p\right\} .
$$

The class $\partial v_{1}$ maps to $s$ in $V(1)_{*} K\left(k u_{p}\right)$ via $\tau_{*}$. The class $1 \in V(1)_{0} K\left(\mathbb{Z}_{p}\right)$ is in the kernel of $\tau_{*}$, because it is $v_{2}$-torsion and there is no torsion class in $V(1)_{0} K\left(k u_{p}\right)$. Let $d \in V(1)_{1} K\left(K U_{p}\right)$ be the class mapping to $1 \in V(1)_{0} K\left(\mathbb{Z}_{p}\right)$ via the connecting homomorphism. Presumably $d$ corresponds to the added unit or the self-equivalence

$$
K U_{p} \stackrel{u}{\longrightarrow} \Sigma^{-2} K U_{p} \stackrel{\simeq}{\longrightarrow} K U_{p}
$$

where $u$ denotes multiplication by the Bott class, and the second map is the Bott equivalence. The class $d$ maps in $V(1)_{1} T H H\left(k u_{p} \mid K U_{p}\right)$ to a class with the same name. In [1, $\S 10$ ] we establish an (additive) isomorphism

$$
V(1)_{*} T H H\left(k u_{p} \mid K U_{p}\right) \cong P_{p-1}(u) \otimes E\left(d, \lambda_{1}\right) \otimes P\left(\mu_{1}\right) .
$$

If this is an isomorphism of algebras, then $j_{*}\left(b_{1}\right) d=j_{*}\left(a_{1}\right)$ holds in $V(1)_{*} T H H\left(k u_{p} \mid K U_{p}\right)$, and lifts to the relation $j_{*}(b) d=j_{*}\left(a_{1}\right)$ in $V(1)_{*} K\left(K U_{p}\right)$. By inspection this determines the structure of $V(1)_{*} K\left(K U_{p}\right)$ as a $P(b)$-module.

Theorem 8.3. Under the hypothesis that there exists a commutative diagram of localization sequences (8.1), and that the isomorphism (8.2) is one of algebras, we have an isomorphism of $P(b)$-modules

$$
\begin{aligned}
V(1)_{*} K\left(K U_{p}\right) \cong & P(b) \otimes E\left(\lambda_{1}, d\right) \oplus P(b) \otimes \mathbb{F}_{p}\left\{\partial \lambda_{1}, \partial b, \partial a_{1}, \partial \lambda_{1} d\right\} \\
& \oplus P(b) \otimes E(d) \otimes \mathbb{F}_{p}\left\{t^{d} \lambda_{1} \mid 0<d<p\right\} \\
& \oplus P(b) \otimes E\left(\lambda_{1}\right) \otimes \mathbb{F}_{p}\left\{\sigma_{n}, \lambda_{2} t^{p^{2}-p} \mid 1 \leqslant n \leqslant p-2\right\} .
\end{aligned}
$$

The class d has degree 1, and the other classes have the degree given in Theorem 8.1 .

Remark 8.4. Consider a complete discrete valuation field $K$ of characteristic zero with perfect residue field $k$ of characteristic $p \geqslant 3$, and let $A$ be its valuation ring. Hesselholt and Madsen [23] compute the $V(0)$-homotopy of $K(A)$ and $K(K)$ by means of the cyclotomic trace. They introduce a relative version of topological cyclic homology, denoted $T C(A \mid K)$, that sits in a localization cofibre sequence

$$
T C(k) \rightarrow T C(A) \rightarrow T C(A \mid K) \rightarrow \Sigma T C(k) .
$$

The computation of $V(0)_{*} T C(A \mid K)$ is achieved by using the rich algebraic structure on the $V(0)$-homotopy groups of the tower $T R^{\bullet}(A \mid K)$, and described in terms of the 
de Rham-Witt complex with log poles

$$
W \cdot \omega^{*}\left(A, A \cap K^{\times}\right),
$$

see [23, Th. C]. Then $V(0)_{*} T C(A)$ can be evaluated by means of the localization sequence. This approach has, in particular, the advantage of avoiding a computation of $V(0)_{*} T R^{\bullet}(A)$, which seems quite intractable.

Continuing the discussion in [1, §10] on a relative trace for $k u_{p}$, and following Lars Hesselholt, one could speculate on the existence of a relative term $T C\left(k u_{p} \mid K U_{p}\right)$ fitting in a localization sequence

$$
T C\left(H \mathbb{Z}_{p}\right) \rightarrow T C\left(k u_{p}\right) \rightarrow T C\left(k u_{p} \mid K U_{p}\right) \rightarrow \Sigma T C\left(H \mathbb{Z}_{p}\right),
$$

through which the trace of diagram (8.1) factorizes. By analogy with the case of complete discrete valuation fields, we expect that a computation of $V(1)_{*} T R^{n}\left(k u_{p} \mid K U_{p}\right)$ should be easier to handle than the computation of $V(1)_{*} T R^{n}\left(k u_{p}\right)$ presented in this paper. In fact, the advantage of such an approach is already apparent when comparing

$$
V(1)_{*} T R^{1}\left(k u_{p} \mid K U_{p}\right)=V(1)_{*} T H H\left(k u_{p} \mid K U_{p}\right)
$$

in (8.2) with $V(1)_{*} T H H\left(k u_{p}\right)$ in (4.1), and is also confirmed by partial, hypothetical computations of $V(1)_{*} T R^{n}\left(\ell_{p} \mid L_{p}\right)$ and $V(1)_{*} T R^{n}\left(k u_{p} \mid K U_{p}\right)$ by Lars Hesselholt (private communication) and the author.

Acknowledgements. This paper is part of my Habilitation thesis written at the University of Bonn. I thank Stefan Schwede, Carl-Friedrich Bödigheimer, Gérald Gaudens and my other colleagues in Bonn for their friendly support. I thank Birgit Richter, Bjørn Dundas, John Greenlees, Lars Hesselholt, Christian Schlichtkrull and Neil Strickland for interesting conversations related to this project. This paper builds on the results of [3], and uses many techniques and ideas that I learned from John Rognes. I am very grateful to him for his help and his generosity. Finally, I thank the referee for his many useful suggestions.

\section{REFERENCES}

[1] Ch. Ausoni, Topological Hochschild homology of connective complex K-theory, Amer. J. Math. 127 (2005), no. 6, 1261-1313.

[2] Ch. Ausoni, B. I. Dundas, and J. Rognes, Divisibility of the Dirac magnetic monopole as a two-vector bundle over the three-sphere, Doc. Math. 13 (2008), 795-801.

[3] Ch. Ausoni and J. Rognes, Algebraic K-theory of topological K-theory, Acta Math. 188 (2002), no. 1, 1-39.

[4] Ch. Ausoni and J. Rognes, The chromatic red-shift in algebraic K-theory, Enseign. Math. (2) 54 (2008), 9-11.

[5] Ch. Ausoni and J. Rognes, Rational algebraic K-theory of topological K-theory, available at arxiv:math.KT/0708.2160

[6] N. A. Baas, B. I. Dundas, B. Richter, and J. Rognes, Stable bundles over rig categories, available at arxiv:math.KT/0909.1742.

[7] N. A. Baas, B. I. Dundas, and J. Rognes, Two-vector bundles and forms of elliptic cohomology, Topology, geometry and quantum field theory, London Math. Soc. Lecture Note Ser., vol. 308, Cambridge Univ. Press, Cambridge, 2004, pp. 18-45.

[8] A. J. Blumberg and M. A. Mandell, The localization sequence for the algebraic K-theory of topological K-theory, Acta Math. 200 (2008), no. 2, 155-179.

[9] M. Bökstedt, B. Bruner, S. Lunøe-Nielsen, and J. Rognes, On cyclic fixed points of spectra, available at arxiv:math.AT/0712.3476.

[10] M. Bökstedt, G. Carlsson, R. Cohen, T. Goodwillie, W. C. Hsiang, and I. Madsen, On the algebraic K-theory of simply connected spaces, Duke Math. J. 84 (1996), no. 3, 541-563. 
[11] M. Bökstedt, W. C. Hsiang, and I. Madsen, The cyclotomic trace and algebraic K-theory of spaces, Invent. Math. 111 (1993), no. 3, 465-539.

[12] M. Bökstedt and I. Madsen, Topological cyclic homology of the integers, Astérisque 226 (1994), 7-8, 57-143. $K$-theory (Strasbourg, 1992).

[13] M. Bökstedt and I. Madsen, Algebraic K-theory of local number fields: the unramified case, Prospects in topology (Princeton, NJ, 1994), 1995, pp. 28-57.

[14] A. Borel, Stable real cohomology of arithmetic groups, Ann. Sci. École Norm. Sup. (4) 7 (1974), $235-272$.

[15] W. Browder, Algebraic K-theory with coefficients $\mathbb{Z} / p$, Geometric applications of homotopy theory (Proc. Conf., Evanston, Ill., 1977), I, Lecture Notes in Math., vol. 657, Springer, Berlin, 1978, pp. 40-84.

[16] R. R. Bruner and J. Rognes, Differentials in the homological homotopy fixed point spectral sequence, Algebr. Geom. Topol. 5 (2005), 653-690.

[17] H. Cartan, Séminaire Henri Cartan de l'Ecole Normale Supérieure, 1954/1955. Algèbres d'Eilenberg-Mac Lane et homotopie, Secrétariat mathématique, 11 rue Pierre Curie, Paris, 1955 (French).

[18] B. I. Dundas, Relative K-theory and topological cyclic homology, Acta Math. 179 (1997), no. 2, 223-242.

[19] W. G. Dwyer and E. M. Friedlander, Algebraic and étale K-theory, Trans. Amer. Math. Soc. 292 (1985), no. 1, 247-280.

[20] A. D. Elmendorf, I. Kriz, M. A. Mandell, and J. P. May, Rings, modules, and algebras in stable homotopy theory, Mathematical Surveys and Monographs, vol. 47, American Mathematical Society, Providence, RI, 1997. With an appendix by M. Cole.

[21] P. G. Goerss and M. J. Hopkins, Moduli spaces of commutative ring spectra, Structured ring spectra, London Math. Soc. Lecture Note Ser., vol. 315, Cambridge Univ. Press, Cambridge, 2004, pp. 151200.

[22] L. Hesselholt and I. Madsen, On the K-theory of finite algebras over Witt vectors of perfect fields, Topology 36 (1997), no. 1, 29-101.

[23] L. Hesselholt and I. Madsen, On the K-theory of local fields, Ann. of Math. (2) 158 (2003), no. 1, $1-113$.

[24] M. J. Hopkins, Algebraic topology and modular forms, Proceedings of the International Congress of Mathematicians, Vol. I (Beijing, 2002), 2002, pp. 291-317.

[25] M. J. Hopkins and J. H. Smith, Nilpotence and stable homotopy theory. II, Ann. of Math. (2) 148 (1998), no. 1, 1-49.

[26] M. Hovey, B. Shipley, and J. Smith, Symmetric spectra, J. Amer. Math. Soc. 13 (2000), no. 1, 149-208.

[27] J.-L. Loday, Cyclic homology, Grundlehren der Mathematischen Wissenschaften, vol. 301, SpringerVerlag, 1998.

[28] S. Lunøe-Nielsen, The Segal conjecture for topological Hochschild homology of commutative Salgebras, University of Oslo Ph.D. thesis (2005).

[29] I. Madsen and Ch. Schlichtkrull, The circle transfer and K-theory, Geometry and topology: Aarhus (1998), Contemp. Math., vol. 258, 2000, pp. 307-328.

[30] J. P. May, $E_{\infty}$ ring spaces and $E_{\infty}$ ring spectra, Lecture Notes in Mathematics, Vol. 577, SpringerVerlag, 1977. With contributions by Frank Quinn, Nigel Ray, and Jørgen Tornehave.

[31] J. P. May, What precisely are $E_{\infty}$ ring spaces and $E_{\infty}$ ring spectra?, New topological contexts for Galois theory and algebraic geometry (BIRS 2008), Geom. Topol. Monogr., vol. 16, 2009, pp. 215282.

[32] J. E. McClure and R. E. Staffeldt, On the topological Hochschild homology of bu, I, Amer. J. Math. 115 (1993), no. 1, 1-45.

[33] J. Milnor, The Steenrod algebra and its dual, Ann. of Math. (2) 67 (1958), 150-171.

[34] S. A. Mitchell, On the Lichtenbaum-Quillen conjectures from a stable homotopy-theoretic viewpoint, Algebraic topology and its applications, Math. Sci. Res. Inst. Publ., vol. 27, Springer, New York, 1994, pp. 163-240.

[35] S. Oka, Multiplicative structure of finite ring spectra and stable homotopy of spheres, Algebraic topology, Aarhus 1982 (Aarhus, 1982), Lecture Notes in Math., vol. 1051, Springer, Berlin, 1984, pp. $418-441$. 
[36] D. C. Ravenel and W. S. Wilson, The Morava K-theories of Eilenberg-Mac Lane spaces and the Conner-Floyd conjecture, Amer. J. Math. 102 (1980), no. 4, 691-748.

[37] J. Rognes, Trace maps from the algebraic K-theory of the integers (after Marcel Bökstedt), J. Pure Appl. Algebra 125 (1998), no. 1-3, 277-286.

[38] J. Rognes, Galois extensions of structured ring spectra, Mem. Amer. Math. Soc. 192 (2008), no. 898, $1-97$.

[39] Ch. Schlichtkrull, Units of ring spectra and their traces in algebraic K-theory, Geom. Topol. 8 (2004), 645-673.

[40] V. Snaith, Unitary K-homology and the Lichtenbaum-Quillen conjecture on the algebraic K-theory of schemes, Algebraic topology, Aarhus 1982 (Aarhus, 1982), Lecture Notes in Math., vol. 1051, Springer, Berlin, 1984, pp. 128-155.

[41] R. W. Thomason, Algebraic K-theory and étale cohomology, Ann. Sci. École Norm. Sup. (4) 18 (1985), no. 3, 437-552.

[42] H. Toda, On spectra realizing exterior parts of the Steenrod algebra, Topology 10 (1971), 53-65.

[43] S. Tsalidis, Topological Hochschild homology and the homotopy descent problem, Topology 37 (1998), no. 4, 913-934.

[44] F. Waldhausen, Algebraic K-theory of topological spaces. I, Algebraic and geometric topology (Proc. Sympos. Pure Math., Stanford Univ., Stanford, Calif., 1976), Part 1, 1978, pp. 35-60.

Mathematical Institute, University of Bonn, Germany

E-mail address: ausoni@math.uni-bonn.de 